Supporting information for

\title{
Fullerene-Assisted Photoinduced Charge Transfer of Single-Walled Carbon Nanotubes through a Flavin-Helix
}

\author{
Mehdi Mollahosseini, ${ }^{1,2}$ Erandika Karunaratne, ${ }^{1,2}$ George N. Gibson, ${ }^{3}$ Jose A. Gascón, ${ }^{2}$ \\ and Fotios Papadimitrakopoulos, ${ }^{1,2 *}$ \\ ${ }^{1}$ Nanomaterials Optoelectronics Laboratory (NOEL), Polymer Program, Institute of Materials Science, \\ ${ }^{2}$ Dept. of Chemistry, ${ }^{3}$ Dept. of Physics, University of Connecticut, Storrs, Connecticut 06269, USA \\ *Corresponding author: papadim@uconn.edu
}

Table of content

A. Quantification of fragment contributions between the isoalloxazine, $\mathrm{C}_{60}$, and flexible chain moieties of $\mathrm{FC}_{60}$ in isolated and helix-incorporated environments (Figures S1-S4 and Tables S1-S17)

B. NMR characterization of Scheme 1 intermediates (i.e. (2), (3), (4) and (5)), along with $\mathrm{FC}_{60}$ (1) (Figures S5-S11 and Table S18)

C. Unsuccessful synthesis routes for the realization of $\mathrm{FC}_{60}$ (1).

C.1. Unsuccessful route 1 (Scheme S1 and Figures S12-S13)

C.2. Unsuccessful route 2 (Scheme $\mathbf{S 2}$ and Figure S14).

C.3. Unsuccessful route 3 (Scheme S3 and Figures S15)

C.4. Unsuccessful route 4 (Scheme S4 and Figures S16)

D. FTIR determination of isoalloxazine/ $\mathrm{C}_{60}$ charge transfer interactions in $\mathrm{FC}_{60}$ (Figures S17).

E. $(n, m)$-characterization of FC12-dispersed SWNTs in the presence and absence of $\mathrm{FC}_{60}$ and $\mathrm{C}_{60}$ (Figures S18-S19 and Tables S19)

F. Demonstration and quantification of $\mathrm{FC}_{60}$ incorporation within $\mathrm{FC} 12$ helices (Figures S20-S22)

G. Spectroscopic changes in FC12-wrapped SWNTs with increasing incorporation of $\mathrm{FC}_{60}$ (Figures S23-S26 and Tables S20-S21).

H. Transient spectroscopy of SWNTs wrapped with FC12 and a 3/97 \% molar mixture of $\mathrm{FC}_{60} / \mathrm{FC} 12$ (Figures S27-S28) S42-S43

I. Cited References S44 


\section{A. Quantification of fragment contributions between the isoalloxazine, $\mathbf{C}_{60}$, and flexible chain moieties of $\mathrm{FC}_{60}$ in isolated and helix-incorporated environments}

As explained in the Experimental Section of the main text, molecular mechanics (MM) were used to extract intra- and inter-molecular interactions of the extended and bent conformations of $\mathrm{FC}_{60}$ (Figure $\mathrm{S} 1$ ). For all local minima, the flexible aliphatic chain of $\mathrm{FC}_{60}$ bends and wraps around the $\mathrm{C}_{60}$ moiety. Table $\mathrm{S} 1$ illustrates the energy contributions of the four most stable $\mathrm{FC}_{60}$ bent structures obtained from quenched dynamics, along with the net energy gain afforded via bending. Bending dramatically shortens the distance between the $\mathrm{C}_{60}$ and the isoalloxazine ring (as shown in Figure S1b\&c) down to 2.9-3.0 $\AA$ (Table S2), which facilitates $\pi-\pi$ stacking interactions.

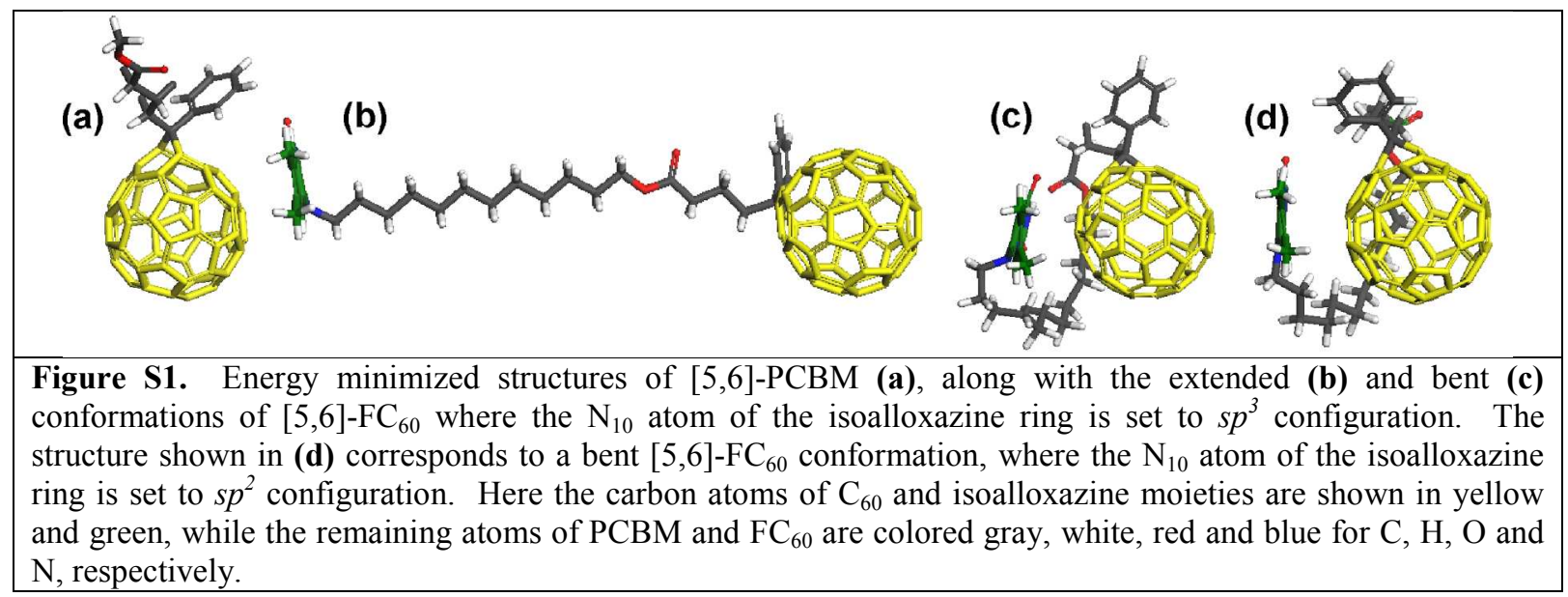

Table S1. Energy contributions (in $\mathrm{kcal} / \mathrm{mol}$ ) for the energy-minimized extended- and bent-conformations of $\mathrm{FC}_{60}$, along with the net energy gain (in negative) afforded via bending for each of the four $\mathrm{FC}_{60}$ configurations studied.

\begin{tabular}{|c|c|c|c|c|c|c|c|c|}
\hline \multirow{2}{*}{$\mathrm{FC}_{60}$ configuration } & \multicolumn{4}{|c|}{ Bonding Energy (kcal/mol) } & \multicolumn{3}{|c|}{ Nonbonding Energy $(\mathrm{kcal} / \mathrm{mol})$} & \multirow{2}{*}{$\begin{array}{c}\boldsymbol{E}_{\text {total }}^{*} \\
(\mathrm{kcal} / \mathrm{mol})\end{array}$} \\
\hline & Bond & Angle & Torsion & Inversion & vdW & Electrostatic & H-bond & \\
\hline$[5,6]-s p^{3}$-bent & 45.0 & 145.1 & 449.0 & 385.9 & 203.6 & -20.8 & -3.4 & 1204.4 \\
\hline$[5,6]-s p^{3}$-extended & 45.4 & 142.0 & 445.5 & 386.0 & 229.1 & -11.5 & 0.0 & 1236.5 \\
\hline $\begin{array}{c}{[5,6]-s p^{3} \text {-net gain }} \\
\text { (bent-extended) }\end{array}$ & -0.4 & 3.1 & 3.5 & -0.1 & -25.5 & -9.3 & -3.4 & $-32.1^{*}$ \\
\hline$[5,6]-s p^{2}-b e n t$ & 46.1 & 143.4 & 447.5 & 385.8 & 208.5 & -20.1 & -3.3 & 1207.9 \\
\hline$[5,6]-s p^{2}$-extended & 46.7 & 140.6 & 445.3 & 385.9 & 234.8 & -13.7 & 0.0 & 1239.6 \\
\hline $\begin{array}{c}{[5,6]-s p^{2} \text {-net gain }} \\
(\text { bent-extended })\end{array}$ & -0.6 & 2.8 & 2.2 & -0.1 & -26.3 & -6.4 & -3.3 & -31 \\
\hline$[6,6]-s p^{3}$-bent & 35.1 & 278.8 & 421.2 & 385.7 & 176.1 & -18.6 & -2.6 & 1275.7 \\
\hline$[6,6]-s p^{3}$-extended & 35.0 & 275.2 & 418.6 & 385.5 & 202.1 & -11.7 & 0.0 & 1304.7 \\
\hline $\begin{array}{c}{[6,6]-s p^{3} \text {-net gain }} \\
\text { (bent-extended) }\end{array}$ & 0.1 & 3.6 & 2.6 & 0.2 & -26.0 & -6.9 & -2.6 & $-29.0^{*}$ \\
\hline$[6,6]-s p^{2}$-bent & 36.5 & 276.3 & 422.0 & 385.5 & 179.2 & -14.4 & 0.0 & 1285.1 \\
\hline$[6,6]-s p^{2}$-extended & 36.4 & 274.0 & 418.3 & 385.5 & 207.9 & -12.5 & 0.0 & 1309.6 \\
\hline $\begin{array}{c}{[6,6]-s p^{2} \text {-net gain }} \\
\text { (bent-extended) }\end{array}$ & 0.1 & 2.3 & 3.7 & 0.0 & -28.7 & --1.9 & 0.0 & $-24.5^{*}$ \\
\hline
\end{tabular}

* The $E_{\text {total }}$ difference between the bent and extended $\mathrm{FC}_{60}$ conformation is herein defined as molecular mechanics (MM)-gain total $\left(\boldsymbol{E}_{M M \text {-gain total }}\right)$. 
Table S2. Average distances between $\mathrm{C}_{60}$ and isoalloxazine ring for different bent $\mathrm{FC}_{60}$ configurations.

\begin{tabular}{|c|c|}
\hline $\mathbf{F C}_{\mathbf{6 0}}$ configuration & Distance $(\boldsymbol{\AA})$ \\
\hline$[5,6]-s p^{3}-\mathrm{FC}_{60}$ & 3.0 \\
\hline$[5,6]-s p^{2}-\mathrm{FC}_{60}$ & 3.0 \\
\hline$[6,6]-s p^{3}-\mathrm{FC}_{60}$ & 2.9 \\
\hline$[6,6]-s p^{2}-\mathrm{FC}_{60}$ & 3.0 \\
\hline
\end{tabular}

Nonbonding interactions between the three distinct moieties of $\mathrm{FC}_{60}$ (i.e. isoalloxazine, $\mathrm{C}_{60}$ and flexible chain) play an important role in stabilizing its bent conformation. To quantify these pair interactions, namely:

(a) flexible chain with $\mathrm{C}_{60}$;

(b) isoalloxazine with $\mathrm{C}_{60}$; and

(c) flexible chain with isoalloxazine ring,

the $\mathrm{FC}_{60}$ was appropriately fragmented before obtaining single-point energies of each fragment. The point where the $\mathrm{C}_{60}$ moiety was separated from the alkyl chain was found crucial in order to afford additive contributions between the van der Waals and electrostatic interactions. The blue arrow in Figure S2 indicates the position where the flexible chain and the $\mathrm{C}_{60}$ moieties were fragmented for both $[5,6]$ and $[6,6]$ configurations of $\mathrm{FC}_{60}$. Fragmentation between the isoalloxazine and the flexible chain took place immediately after the $\mathrm{N}_{10}$ nitrogen of the ring (shown by the red arrow).

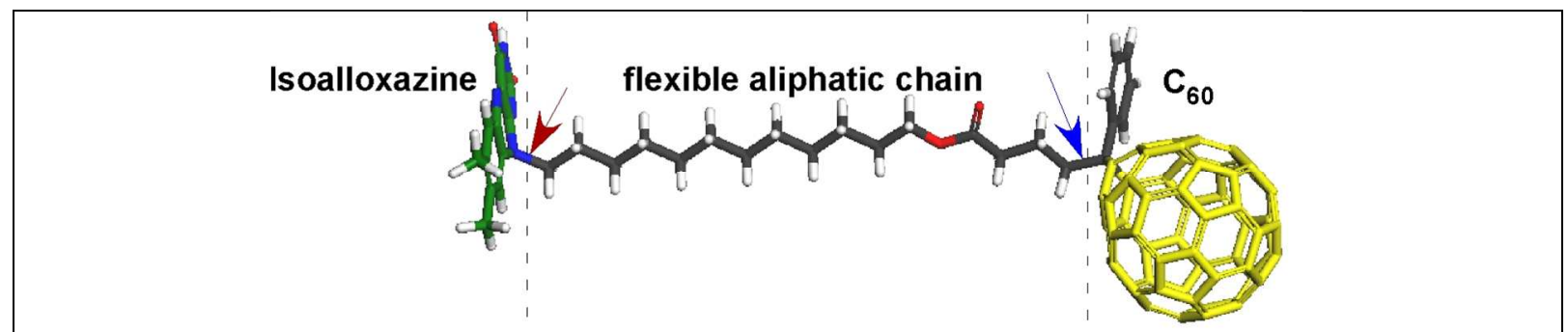

Figure S2. Blue and red arrows indicate the bonds where the [5,6] and $[6,6]$ configurations of $\mathrm{FC}_{60}$ were fragmented in order to calculate single-point fragment energies.

The energy of each fragment can be expressed in terms of its bonding and nonbonding interactions,

$$
E_{\text {frag }}=E_{\text {bonding }}+E_{\text {self-nonbonding }}
$$

where, the "self-nonbonding" term excludes interactions with other nearby fragments. Similarly, the energy of a pair of fragments can be expressed as,

$$
E_{\text {frag1+frag2 }}=E_{\text {bonding }}+E_{\text {self-nonbonding }}+E_{\text {pair-nonbonding }}
$$

where, the "pair-nonbonding" term defines the interactions between fragments 1 and 2 . Equations (1) and (2) affords the determination of pair interactions between fragment pairs (a), (b), and (c) described above. For example, the pair interactions between the flexible chain (abbreviated as "chain") and $\mathrm{C}_{60}$ can be assessed by,

$$
E_{\text {pair interaction }\left(\text { chain- } C_{60}\right)}=E_{\text {pair bonding }\left(\text { chain- } C_{60}\right)}+E_{\text {pair-nonbonding }\left(\text { chain- } C_{60}\right)}=E_{\left(\text {chain }+C_{60}\right)}-\left(E_{\text {chain }}+E_{C_{60}}\right)
$$

where, $E_{\left(\text {chain }+C_{60}\right)}$ is obtained by eq. 2 , while $E_{\text {chain }}$ and $E_{C_{60}}$ by eq. 1 . This pair interaction energy term mostly describes the nonbonding interactions between the two fragments along with some bonding energy contributions that arise from bond formation between the respective fragments 
(i.e. chain- $\mathrm{C}_{60}$ and chain-isoalloxazine). Similar formulae were employed to assess the chainisoalloxazine (abbreviated as "isoall") and isoalloxazine- $\mathrm{C}_{60}$ pair-interactions.

Here it is important to stress that eq. 2 double counts the $E_{\text {bonding }}$ and the $E_{\text {self-nonbonding }}$ terms. While both of these terms are canceled out in eq. 3, this is not true for the direct energy assessment of a given pair. Correspondingly, eq. 4 provides the total energy for a pair of two fragments

$$
E_{(\text {frag } 1+\text { frag } 2)}^{\prime}=E_{\text {frag } 1} / 2+E_{\text {frag } 2} / 2+E_{\text {pair-nonbonding (fragment1-fragment2) }}
$$

where $E_{\text {fragment } 1}$ and $E_{\text {fragment } 2}$ come from eq. 1, while $E_{\text {pair-nonbonding (fragment1-fragment2) }}$ originates from eq. 3. For example, the energy for each of the three pairs is shown below.

$$
\begin{gathered}
E_{\left(\text {chain }+C_{60}\right)}^{\prime}=E_{\text {chain }} / 2+E_{C_{60}} / 2+E_{\text {pair-nonbonding }\left(\text { chain- } C_{60}\right)} \\
E_{\left(\text {isoall }+C_{60}\right)}^{\prime}=E_{\text {isoall }} / 2+E_{C_{60}} / 2+E_{\text {pair-nonbonding (isoall-C } \left.C_{60}\right)} \\
E_{(\text {chain }+ \text { isoall })}^{\prime}=E_{\text {chain }} / 2+E_{\text {isoall }} / 2+E_{\text {pair-nonbonding (chain-isoall) }}
\end{gathered}
$$

Similar formalism was applied for the extended $\mathrm{FC}_{60}$ conformations. The energy gain afforded by chain bending was calculated for each pair by subtracting the extended from the bent energy of the pair.

$$
E_{\text {gain }}=E_{\text {bend }}-E_{\text {extended }}
$$

Eq. 5 affords the determination of $E_{\text {gain }}$ for the aforementioned three pairs. The validity of such fragmentation calculation can be checked by how close is the sum of the three $E_{\text {gain }}$ terms (herein defined as $\left.E_{\text {fragment-gain total }}\right)$ in comparison with the total energy gain from bending ( $\left.E_{M M \text {-gain total }}\right)$ obtained from Table S1.

$\underline{\text { Isolated } \mathbf{F C}_{60}}$ : : Table $\mathrm{S} 3$ presents the individual fragment energies $\left(E_{\text {frag }}\right)$ obtained for all four $\mathrm{FC}_{60}$ configurations in both their extended and bent conformations. As described in eq. 1, these fragment energies are composed of bonding and self-nonbonding terms. Similarly, Table S4 shows the energies of fragment-pairs (i.e. flexible chain with $\mathrm{C}_{60}$, isoalloxazine with $\mathrm{C}_{60}$, and flexible chain with isoalloxazine) for all $\mathrm{FC}_{60}$ configurations. As described in eq. 2 , these fragment-pair energies are composed of bonding and nonbonding terms, the latter of which comprises from both self- and pair-nonbonding contributions.

Using eq. 3 and the energy values of Tables S3 and S4, the pair interactions between two fragments, which mostly comprises from the $E_{\text {pair-nonbonding, were calculated and tabulated in }}$ Table S5. Table S6 shows the recalculated fragment-pair energy $E_{(\text {frag1+frag2) }}$ by employing eq. 4 , which accounts for double counting the individual $E_{\text {bonding }}$ and $E_{\text {self-nonbonding }}$ fragment terms.

Table S7 depicts the energy gain $\left(E_{\text {gain }}\right)$ calculated by eq. 5 for the three fragment-pairs of $\mathrm{FC}_{60}$ at each of the four $\mathrm{FC}_{60}$ configurations studied. The summation of all three fragment-pair energy contributions is shown in Table S8 with the sum of the three $E_{\text {gain }}$ terms shown as $E_{\text {fragment-gain total }}$. The $E_{\text {fragment-gain total }}$ values for all four $\mathrm{FC}_{60}$ configurations are in close proximity (within $0.3 \%)$ of the total energy gain $\left(E_{M M \text {-gain total }}\right)$ obtained from Table S1. This validates the aforementioned fragmentation treatment. Moreover, it allows the break down the van der Waals (vdW) and electrostatic (ES) values of Table S1 into non-bonding pair contributions between the three respective fragments.

These contributions are summarized in Table 1 of the main text, where the sum of all bonding terms, vdW, ES, H-bonds and total energy values are extracted from Table S8, while the breakdown of vdW and ES terms in fragment-pair contributions stem from Table S7. 
Table S3. Energy contributions for the three fragments (i.e. flexible chain, $\mathrm{C}_{60}$ and isoalloxazine ring) of the energy-minimized extended- and bent-conformations of $\mathrm{FC}_{60}$ for each of the four $\mathrm{FC}_{60}$ configurations studied. As described in eq. 1, these fragment energies are composed of bonding and self-nonbonding terms.

\begin{tabular}{|c|c|c|c|c|c|c|c|c|c|}
\hline \multirow[t]{2}{*}{$\mathrm{FC}_{60}$ configuration } & \multirow[t]{2}{*}{ Fragment } & \multicolumn{4}{|c|}{ Bonding energy $(\mathrm{kcal} / \mathrm{mol})$} & \multicolumn{3}{|c|}{$\begin{array}{c}\text { Self-nonbonding Energy } \\
(\mathrm{kcal} / \mathrm{mol})\end{array}$} & \multirow{2}{*}{$\begin{array}{c}\boldsymbol{E}_{\text {frag }} \\
(\mathrm{kcal} / \mathrm{mol})\end{array}$} \\
\hline & & Bond & Angle & Torsion & Inversion & VdW & Electrosta & H-bonds & \\
\hline \multirow{3}{*}[5,6]{$-s p^{3}$-bent } & chain & 3.4 & 8.0 & 2.0 & 0.0 & 20.1 & 20.6 & 0.0 & 54.2 \\
\hline & $\mathrm{C}_{60}$ & 34.3 & 131.9 & 443.6 & 385.7 & 167.9 & 1.8 & 0.0 & 1165.2 \\
\hline & isoall & 5.5 & 2.6 & 3.3 & 0.2 & 33.4 & -28.6 & 0.0 & 16.3 \\
\hline \multirow{3}{*}[5,6]{$-s p^{3}$-extended } & chain & 3.1 & 4.1 & 0.1 & 0.0 & 20.4 & 12.0 & 0.0 & 39.8 \\
\hline & $\mathrm{C}_{60}$ & 35.0 & 132.0 & 444.0 & 385.9 & 169.4 & 2.2 & 0.0 & 1168.5 \\
\hline & isoall & 5.5 & 3.0 & 1.6 & 0.0 & 32.8 & -24.5 & 0.0 & 18.5 \\
\hline \multirow{3}{*}{$\begin{array}{c}{[5,6]-s p^{3}-} \\
\text { (bent-extended) }\end{array}$} & chain & 0.2 & 3.9 & 1.8 & 0.0 & -0.2 & 8.6 & 0.0 & 14.4 \\
\hline & $\mathrm{C}_{60}$ & -0.7 & -0.1 & -0.5 & -0.2 & -1.5 & -0.4 & 0.0 & -3.4 \\
\hline & isoall & 0.0 & -0.4 & 1.6 & 0.2 & 0.6 & -4.1 & 0.0 & -2.2 \\
\hline \multirow{3}{*}[5,6]{$-s p^{2}$-bent } & chain & 3.3 & 7.2 & 1.8 & 0.0 & 20.1 & 19.8 & 0.0 & 52.2 \\
\hline & $\mathrm{C}_{60}$ & 34.0 & 132.0 & 443.6 & 385.7 & 167.9 & 1.8 & 0.0 & 1164.9 \\
\hline & isoall & 6.7 & 2.8 & 1.1 & 0.1 & 34.4 & -27.8 & 0.0 & 17.4 \\
\hline \multirow{3}{*}[5,6]{$-s p^{2}$-extended } & chain & 2.9 & $\begin{array}{ll}4.4 \\
\end{array}$ & 0.1 & 0.0 & 20.6 & 10.7 & 0.0 & 38.7 \\
\hline & $\mathrm{C}_{60}$ & 35.0 & 132.0 & 444.0 & 385.9 & 169.4 & 1.8 & 0.0 & 1168.2 \\
\hline & isoall & 6.8 & 2.8 & 0.2 & 0.0 & 34.2 & -23.4 & 0.0 & 20.5 \\
\hline \multirow{3}{*}{$\begin{array}{c}{[5,6]-s p^{2}-} \\
\text { (bent-extended) }\end{array}$} & chain & 0.5 & 2.8 & 1.6 & 0.0 & -0.5 & 9.1 & 0.0 & 13.5 \\
\hline & $\mathrm{C}_{60}$ & -1.0 & -0.1 & -0.5 & -0.2 & -1.6 & -0.1 & 0.0 & -3.4 \\
\hline & isoall & -0.1 & 0.1 & 0.9 & 0.1 & 0.2 & -4.4 & 0.0 & -3.1 \\
\hline \multirow{3}{*}[6,6]{$-s p^{3}$-bent } & chain & 3.3 & 7.2 & 0.9 & 0.0 & 18.8 & 21.3 & $\overline{0.0}$ & 51.5 \\
\hline & $\mathrm{C}_{60}$ & 25.3 & 263.9 & 416.3 & 385.5 & 147.1 & 1.7 & 0.0 & 1239.7 \\
\hline & isoall & 5.4 & 2.8 & 2.8 & 0.2 & 33.3 & -28.8 & 0.0 & 15.8 \\
\hline \multirow{3}{*}[6,6]{$-s p^{3}$-extended } & chain & 2.9 & 3.8 & 0.1 & 0.0 & 20.3 & 12.5 & 0.0 & 39.5 \\
\hline & $\mathrm{C}_{60}$ & 25.7 & 263.8 & 416.3 & 385.5 & 146.7 & 1.7 & 0.0 & 1239.6 \\
\hline & isoall & 5.5 & 3.1 & 1.6 & 0.0 & 32.8 & -24.7 & 0.0 & 18.4 \\
\hline \multirow{3}{*}{$\begin{array}{c}{[6,6]-s p^{3}-} \\
\text { (bent-extended) }\end{array}$} & chain & 0.4 & 3.4 & 0.8 & 0.0 & -1.5 & 8.8 & 0.0 & 12.0 \\
\hline & $\mathrm{C}_{60}$ & -0.3 & 0.1 & 0.0 & -0.1 & 0.4 & 0.0 & 0.0 & 0.1 \\
\hline & isoall & -0.1 & -0.3 & 1.2 & 0.2 & 0.5 & -4.1 & 0.0 & -2.7 \\
\hline \multirow{3}{*}[6,6]{$-s p^{2}$-bent } & chain & 3.2 & 6.4 & 2.6 & 0.0 & 20.8 & 20.5 & 0.0 & 53.6 \\
\hline & $\mathrm{C}_{60}$ & 25.3 & 263.8 & 416.2 & 385.5 & 147.1 & 2.0 & 0.0 & 1239.9 \\
\hline & isoall & 6.7 & 2.9 & 1.2 & 0.1 & 34.3 & -23.5 & 0.0 & 21.7 \\
\hline \multirow{3}{*}[6,6]{$-s p^{2}$-extended } & chain & 2.6 & 4.1 & 0.1 & 0.0 & 20.6 & 12.6 & 0.0 & 40.0 \\
\hline & $\mathrm{C}_{60}$ & 25.7 & 263.8 & 416.3 & 385.5 & 146.7 & 1.7 & 0.0 & 1239.6 \\
\hline & isoall & 6.8 & 2.9 & 0.1 & 0.0 & 34.1 & -24.2 & 0.0 & 19.8 \\
\hline \multirow{3}{*}{$\begin{array}{c}{[6,6]-s p^{2}-} \\
\text { (bent-extended) }\end{array}$} & chain & 0.6 & 2.4 & 2.5 & 0.0 & 0.2 & 7.9 & 0.0 & 13.6 \\
\hline & $\mathrm{C}_{60}$ & -0.3 & 0.0 & -0.1 & 0.0 & 0.4 & 0.3 & 0.0 & 0.3 \\
\hline & isoall & -0.1 & 0.0 & 1.1 & 0.1 & 0.2 & 0.7 & 0.0 & 1.9 \\
\hline
\end{tabular}


Table S4. Energy contributions for the three fragment-pairs (i.e. flexible chain with $\mathrm{C}_{60}$, isoalloxazine with $\mathrm{C}_{60}$, and flexible chain with isoalloxazine) of the energy-minimized extended- and bent-conformations of $\mathrm{FC}_{60}$ for each of the four $\mathrm{FC}_{60}$ configurations studied. As described in eq. 2, these fragment-pair energies are composed of bonding and nonbonding terms, the latter of which comprises from both self- and pair-nonbonding contributions.

\begin{tabular}{|c|c|c|c|c|c|c|c|c|c|}
\hline \multirow{2}{*}{$\begin{array}{c}\mathbf{F C}_{60} \\
\text { configuration }\end{array}$} & \multirow{2}{*}{$\underset{\text { pair }}{\text { Fragment }}$} & \multicolumn{4}{|c|}{$\begin{array}{l}\text { Bonding energy } \\
(\mathrm{kcal} / \mathrm{mol})\end{array}$} & \multicolumn{3}{|c|}{$\begin{array}{l}\text { Self-nonbonding and pair- } \\
\text { nonbonding energy }\end{array}$} & \multirow{2}{*}{$\begin{array}{l}\boldsymbol{E}_{\text {fragl } 1 \text { frag } 2} \\
(\mathrm{kcal} / \mathrm{mol})\end{array}$} \\
\hline & & Bond & Angle & Torsion & Inversion & $\mathrm{vdW}$ & Electrostat & H-bonds & \\
\hline \multirow{3}{*}[5,6]{$-s p^{3}$-bent } & chain $/ \mathrm{C}_{60}$ & 38.7 & 141.0 & 445.6 & 385.7 & 182.8 & 22.0 & 0.0 & 1215.8 \\
\hline & $\mathrm{C}_{60} /$ isoall & 39.8 & 134.5 & 446.8 & 385.9 & 188.1 & -26.9 & 0.0 & 1168.2 \\
\hline & chain/isoall & 9.7 & 12.1 & 5.4 & 0.2 & 54.2 & -22.2 & -3.4 & 56.0 \\
\hline \multirow{3}{*}{$\begin{array}{l}{[5,6]-s p^{3}-} \\
\text { extended }\end{array}$} & chain/ $\mathrm{C}_{60}$ & 39.2 & 137.3 & $4 \quad 444.2$ & 385.9 & 193.3 & 14.4 & 0.0 & 1214.3 \\
\hline & $\mathrm{C}_{60} /$ isoall & 40.5 & 135.0 & 445.7 & 386.0 & 202.3 & -22.4 & 0.0 & 1187.1 \\
\hline & chain/isoall & 9.3 & 8.9 & 1.5 & 0.0 & 56.2 & -14.1 & 0.0 & 61.8 \\
\hline \multirow{3}{*}[5,6]{$-s p^{2}$-bent } & chain $/ \mathrm{C}_{60}$ & 38.4 & 140.3 & 445.5 & 385.7 & 181.4 & 20.8 & 0.0 & 1212.2 \\
\hline & $\mathrm{C}_{60} /$ isoall & 40.8 & 134.8 & 444.6 & 385.8 & 189.4 & -25.9 & 0.0 & 1169.5 \\
\hline & chain/isoall & 11.0 & 10.3 & 3.7 & 0.1 & 60.1 & -21.2 & -3.3 & 60.7 \\
\hline \multirow{3}{*}{$\begin{array}{l}{[5,6]-s p^{2}-} \\
\text { extended }\end{array}$} & chain/ $\mathrm{C}_{60}$ & 39.0 & 137.5 & $4 \quad 444.2$ & 385.9 & 193.5 & 12.7 & 0.0 & 1212.9 \\
\hline & $\mathrm{C}_{60} /$ isoall & 41.8 & 134.8 & 444.2 & 385.9 & 203.6 & -21.6 & 0.0 & 1188.7 \\
\hline & chain/isoall & 10.6 & 7.4 & 1.2 & 0.0 & 61.9 & -15.8 & 0.0 & 65.3 \\
\hline \multirow{3}{*}[6,6]{$-s p^{3}$-bent } & chain $/ \mathrm{C}_{60}$ & 28.9 & 274.3 & 418.2 & 385.5 & 156.1 & 22.8 & 0.0 & 1285.7 \\
\hline & $\mathrm{C}_{60} /$ isoall & 30.7 & 266.6 & 419.1 & 385.7 & 165.4 & -27.4 & 0.0 & 1240.2 \\
\hline & chain/isoall & 9.5 & 11.7 & 3.8 & 0.2 & 53.9 & -19.8 & -2.6 & 56.8 \\
\hline \multirow{3}{*}{$\begin{array}{l}{[6,6]-s p^{3}-} \\
\text { extended }\end{array}$} & chain $/ \mathrm{C}_{60}$ & 28.9 & 270.5 & 417.3 & 385.5 & 166.2 & 14.7 & 0.0 & 1283.1 \\
\hline & $\mathrm{C}_{60} /$ isoall & 31.2 & 266.8 & 418.0 & 385.5 & 179.5 & -23.0 & 0.0 & 1258.1 \\
\hline & chain/isoall & 9.1 & 8.5 & 1.4 & 0.0 & 56.2 & -14.0 & 0.0 & 61.3 \\
\hline \multirow{3}{*}[6,6]{$-s p^{2}$-bent } & chain $/ \mathrm{C}_{60}$ & 28.9 & 273.1 & 419.7 & 385.5 & 155.7 & 22.4 & 0.0 & 1285.3 \\
\hline & $\mathrm{C}_{60} /$ isoall & 32.0 & 266.7 & 417.5 & 385.5 & 166.8 & -24.2 & 0.0 & 1244.4 \\
\hline & chain/isoall & 10.8 & 9.6 & 4.9 & 0.1 & 58.8 & -13.7 & 0.0 & 70.5 \\
\hline \multirow{3}{*}{$\begin{array}{l}{[6,6]-s p^{2}-} \\
\text { extended }\end{array}$} & chain $/ \mathrm{C}_{60}$ & 28.6 & 270.8 & 417.3 & 385.5 & 166.5 & 14.8 & 0.0 & 1283.5 \\
\hline & $\mathrm{C}_{60} /$ isoall & 32.5 & 266.7 & 416.5 & 385.5 & 180.8 & -22.5 & 0.0 & 1259.5 \\
\hline & chain/isoall & 10.4 & 7.2 & 1.1 & 0.0 & 62.0 & -14.8 & 0.0 & 66.0 \\
\hline
\end{tabular}


Table S5. Energy contributions of the pair-interactions (as obtained from Tables S3, S4 and eq. 3) for the three fragment-pairs (i.e. flexible chain with $\mathrm{C}_{60}$, isoalloxazine with $\mathrm{C}_{60}$, and flexible chain with isoalloxazine) of the energy-minimized extended- and bent-conformations of $\mathrm{FC}_{60}$ for each of the four $\mathrm{FC}_{60}$ configurations studied. The non-zero bonding energy terms for two of the three pairs (i.e. flexible chain/ $\mathrm{C}_{60}$ and flexible chain/isoalloxazine) originate from bond formation between these two fragments.

\begin{tabular}{|c|c|c|c|c|c|c|c|c|c|}
\hline \multirow{2}{*}{$\begin{array}{c}\mathrm{FC}_{60} \\
\text { configuration }\end{array}$} & \multirow{2}{*}{$\begin{array}{c}\text { Fragment- } \\
\text { pair }\end{array}$} & \multicolumn{4}{|c|}{$\begin{array}{c}\text { Bonding energy } \\
(\mathrm{kcal} / \mathrm{mol})\end{array}$} & \multicolumn{3}{|c|}{$\begin{array}{c}\text { Pair-nonbonding energy } \\
(\mathrm{kcal} / \mathrm{mol})\end{array}$} & \multirow{2}{*}{$\begin{array}{c}E_{\text {pair-interaction }} \\
(\mathrm{kcal} / \mathrm{mol})\end{array}$} \\
\hline & & Bond & Angle & Torsion & Inversion & vdW & Electrostatic & H-bonds & \\
\hline \multirow{3}{*}[5,6]{$-s p^{3}$-bent } & chain $/ \mathrm{C}_{60}$ & 1.1 & 1.0 & 0.0 & 0.0 & -5.2 & -0.4 & 0.0 & -3.5 \\
\hline & isoall/ $\mathrm{C}_{60}$ & 0.0 & 0.0 & 0.0 & 0.0 & -13.3 & 0.0 & 0.0 & -13.2 \\
\hline & chain/isoall & 0.8 & 1.5 & 0.2 & 0.0 & 0.7 & -14.2 & -3.4 & -14.5 \\
\hline \multirow{3}{*}[5,6]{$-s p^{3}$-extended } & chain/ $\mathrm{C}_{60}$ & 1.1 .1 & 1.2 & 0.1 & 0.0 & $\begin{array}{l}.4 \\
\end{array}$ & 0.3 & 0.0 & 6.0 \\
\hline & isoall $/ \mathrm{C}_{60}$ & 0.0 & 0.0 & 0.0 & 0.0 & 0.0 & 0.0 & 0.0 & 0.0 \\
\hline & chain/isoall & 0.7 & 1.7 & -0.3 & 0.0 & 3.0 & -1.6 & 0.0 & 3.5 \\
\hline \multirow{3}{*}[5,6]{$-s p^{2}$-bent } & chain $/ \mathrm{C}_{60}$ & 1.0 & 1.1 & 0.2 & 0.0 & -6.5 & -0.7 & 0.0 & -4.9 \\
\hline & isoall/ $\mathrm{C}_{60}$ & 0.0 & 0.0 & 0.0 & 0.0 & -12.9 & 0.1 & 0.0 & -12.8 \\
\hline & chain/isoall & 1.0 & 0.3 & 0.9 & 0.0 & 5.6 & -13.3 & -3.3 & -8.9 \\
\hline \multirow{3}{*}[5,6]{$-s p^{2}$-extended } & chain/ $\mathrm{C}_{60}$ & 1.1 & 1.2 & 0.1 & 0.0 & 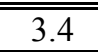 & 0.2 & $\begin{array}{l}0.0 \\
\end{array}$ & $\begin{array}{l}6.0 \\
\end{array}$ \\
\hline & \begin{tabular}{|l|} 
isoall $/ \mathrm{C}_{60}$ \\
\end{tabular} & 0.0 & 0.0 & 0.0 & 0.0 & 0.0 & 0.0 & 0.0 & 0.0 \\
\hline & chain/isoall & 0.9 & 0.3 & 0.9 & 0.0 & 7.2 & -3.1 & 0.0 & 6.2 \\
\hline \multirow{3}{*}[6,6]{$-s p^{3}$-bent } & chain/ $\mathrm{C}_{60}$ & 0.3 & 3.2 & 1.1 & 0.0 & -9.8 & -0.3 & 0.0 & -5.5 \\
\hline & isoall/ $\mathrm{C}_{60}$ & 0.0 & 0.0 & 0.0 & 0.0 & -15.0 & -0.3 & 0.0 & -15.3 \\
\hline & chain/isoall & 0.8 & 1.7 & 0.2 & 0.0 & 1.7 & -12.3 & -2.6 & -10.5 \\
\hline \multirow{3}{*}[6,6]{$-s p^{3}$-extended } & chain/ $\mathrm{C}_{60}$ & 0.3 & 3.0 & 0.9 & 0.0 & -0.8 & 0.6 & 0.0 & 3.9 \\
\hline & \begin{tabular}{|l|} 
isoall $/ \mathrm{C}_{60}$ \\
\end{tabular} & 0.0 & 0.0 & 0.0 & 0.0 & 0.0 & 0.0 & 0.0 & 0.0 \\
\hline & chain/isoall & 0.7 & 1.7 & -0.3 & 0.0 & 3.1 & -1.8 & 0.0 & 3.3 \\
\hline \multirow{3}{*}[6,6]{$-s p^{2}$-bent } & chain $/ \mathrm{C}_{60}$ & 0.3 & 2.9 & 0.9 & 0.0 & -12.1 & -0.1 & 0.0 & -8.1 \\
\hline & isoall/ $\mathrm{C}_{60}$ & 0.0 & 0.0 & 0.0 & 0.0 & -14.6 & -2.6 & 0.0 & -17.2 \\
\hline & chain/isoall & 0.9 & 0.3 & 1.0 & 0.0 & 3.7 & -10.7 & 0.0 & -4.7 \\
\hline \multirow{3}{*}[6,6]{$-s p^{2}$-extended } & chain $/ \mathrm{C}_{60}$ & 0.3 & 3.0 & 0.9 & 0.0 & -0.8 & 0.6 & 0.0 & 3.9 \\
\hline & \begin{tabular}{|l|} 
isoall $/ \mathrm{C}_{60}$ \\
\end{tabular} & 0.0 & 0.0 & 0.0 & 0.0 & 0.0 & 0.0 & 0.0 & 0.0 \\
\hline & chain/isoall & 0.9 & 0.3 & 0.9 & 0.0 & 7.3 & -3.2 & 0.0 & 6.2 \\
\hline
\end{tabular}


Table S6. Recalculated energy contributions (according to eq. 4) of the three fragment-pairs (i.e. flexible chain with $\mathrm{C}_{60}$, isoalloxazine with $\mathrm{C}_{60}$, and flexible chain with isoalloxazine) of the energy-minimized extended- and bentconformations of $\mathrm{FC}_{60}$ for each of the four $\mathrm{FC}_{60}$ configurations studied. The recalculated fragment-pair energies account for double counting of both $E_{\text {bonding }}$ and $E_{\text {self-nonbonding }}$ terms.

\begin{tabular}{|c|c|c|c|c|c|c|c|c|c|}
\hline \multirow{2}{*}{$\begin{array}{c}\mathbf{F C}_{60} \\
\text { configuration }\end{array}$} & \multirow{2}{*}{$\begin{array}{c}\text { Fragment- } \\
\text { pair }\end{array}$} & \multicolumn{4}{|c|}{$\begin{array}{l}\text { Bonding energy } \\
(\mathrm{kcal} / \mathrm{mol})\end{array}$} & \multicolumn{3}{|c|}{\begin{tabular}{|c|} 
Self-nonbonding and pair- \\
nonbonding energy $(\mathrm{kcal} / \mathrm{mol})$
\end{tabular}} & \multirow{2}{*}{$\begin{array}{l}E_{\text {fragl+frag } 2} \\
(\mathrm{kcal} / \mathrm{mol})\end{array}$} \\
\hline & & Bond & Angle & Torsion & Inversion & vdW & Electrostatic & H-bonds & \\
\hline \multirow{3}{*}[5,6]{$-s p^{3}$-bent } & chain $/ \mathrm{C}_{60}$ & 19.9 & 71.0 & 222.8 & 192.9 & 88.8 & 10.8 & 0.0 & 606.2 \\
\hline & isoall $/ \mathrm{C}_{60}$ & 19.9 & 67.3 & 223.4 & 193.0 & 87.4 & -13.4 & 0.0 & 577.5 \\
\hline & chain/isoall & 5.2 & 6.8 & 2.8 & 0.1 & 27.4 & -18.2 & -3.4 & 20.8 \\
\hline \multirow{3}{*}[5,6]{$-s p^{3}$-extended } & chain $/ \mathrm{C}_{60}$ & 20.2 & \begin{tabular}{|l|}
69.2 \\
\end{tabular} & 222.1 & 193.0 & 98.4 & 7.3 & 0.0 & 610.2 \\
\hline & isoall/ $\mathrm{C}_{60}$ & 20.3 & 67.5 & 222.8 & 193.0 & 101.1 & -11.2 & 0.0 & 593.5 \\
\hline & chain/isoall & 5.0 & 5.3 & 0.6 & 0.0 & 29.6 & -7.8 & 0.0 & 32.7 \\
\hline \multirow{3}{*}[5,6]{$-s p^{2}$-bent } & chain/ $\mathrm{C}_{60}$ & 19.7 & 70.7 & 222.8 & 192.8 & 87.4 & 10.1 & 0.0 & 603.6 \\
\hline & isoall $/ \mathrm{C}_{60}$ & 20.4 & 67.4 & 222.3 & 192.9 & 88.2 & -12.9 & 0.0 & 578.3 \\
\hline & chain/isoall & 6.0 & 5.3 & 2.3 & 0.1 & 32.8 & -17.3 & -3.3 & 25.9 \\
\hline \multirow{3}{*}[5,6]{$-s p^{2}$-extended } & chain/ $\mathrm{C}_{60}$ & 20.0 & 69.4 & 222.1 & 193.0 & $\begin{array}{l}98.5 \\
\end{array}$ & (6.5 & "0.0 & 609.4 \\
\hline & isoall/ $\mathrm{C}_{60}$ & 20.9 & 67.4 & 222.1 & 193.0 & 101.8 & -10.8 & 0.0 & 94.3 \\
\hline & chain/isoall & 5.8 & 3.8 & 1.0 & 0.0 & 34.6 & -9.4 & 0.0 & 35.8 \\
\hline \multirow{3}{*}[6,6]{$-s p^{3}$-bent } & chain $/ \mathrm{C}_{60}$ & 14.6 & 138.7 & 209.6 & 192.7 & 73.1 & 11.3 & 0.0 & 640.1 \\
\hline & isoall/ $\mathrm{C}_{60}$ & 15.4 & 133.3 & 209.5 & 192.8 & 75.2 & -13.8 & 0.0 & 612.5 \\
\hline & chain/isoall & 5.1 & 6.7 & 2.0 & 0.1 & 27.8 & -16.0 & -2.6 & 23.2 \\
\hline \multirow{3}{*}[6,6]{$-s p^{3}$-extended } & chain $/ \mathrm{C}_{60}$ & 14.6 & 136.7 & 209.1 & 192.8 & 82.7 & 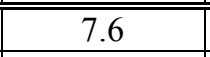 & 0.0 & 643.5 \\
\hline & isoall $/ \mathrm{C}_{60}$ & 15.6 & 133.4 & 209.0 & 192.8 & 89.8 & -11.5 & 0.0 & 629.0 \\
\hline & chain/isoall & 4.9 & 5.1 & 0.5 & 0.0 & 29.7 & -7.9 & 0.0 & 32.3 \\
\hline \multirow{3}{*}[6,6]{$-s p^{2}$-bent } & chain $/ \mathrm{C}_{60}$ & 14.6 & 138.0 & 210.3 & 192.7 & 71.8 & 11.1 & 0.0 & 638.6 \\
\hline & isoall/ $\mathrm{C}_{60}$ & 16.0 & 133.3 & 208.7 & 192.8 & 76.1 & -13.4 & 0.0 & 613.6 \\
\hline & chain/isoall & 5.9 & 5.0 & 3.0 & 0.0 & 31.2 & -12.2 & 0.0 & 32.9 \\
\hline \multirow{3}{*}[6,6]{$-s p^{2}$-extended } & chain $/ \mathrm{C}_{60}$ & 14.5 & \begin{tabular}{|l|}
136.9 \\
\end{tabular} & 209.1 & 192.8 & 82.8 & 7.7 & 0.0 & 643.7 \\
\hline & isoall/ $/ \mathrm{C}_{60}$ & 16.2 & 133.3 & 208.2 & 192.8 & 90.4 & -11.3 & 0.0 & 629.7 \\
\hline & chain/isoall & 5.7 & 3.7 & 1.0 & 0.0 & 34.6 & -9.0 & 0.0 & 36.1 \\
\hline
\end{tabular}


Table S7. Calculated energy gain from bending (according to eq. 5) for the three fragment-pairs (i.e. flexible chain with $\mathrm{C}_{60}$, isoalloxazine with $\mathrm{C}_{60}$, and flexible chain with isoalloxazine) for each of the four $\mathrm{FC}_{60}$ configurations studied.

\begin{tabular}{|c|c|c|c|c|c|c|c|c|c|}
\hline \multirow{2}{*}{$\begin{array}{c}\mathrm{FC}_{60} \\
\text { configuration }\end{array}$} & \multirow{2}{*}{$\begin{array}{c}\text { Fragment- } \\
\text { pair }\end{array}$} & \multicolumn{4}{|c|}{$\begin{array}{c}\text { Bonding energy } \\
(\mathrm{kcal} / \mathrm{mol})\end{array}$} & \multicolumn{3}{|c|}{ Nonbonding energy $(\mathrm{kcal} / \mathrm{mol})$} & \multirow{2}{*}{$\begin{array}{c}\boldsymbol{E}_{\text {gain }} \\
(\mathrm{kcal} / \mathrm{mol})\end{array}$} \\
\hline & & Bond & Angle & Torsion & Inversion & vdW & Electrostati & H-bonds & \\
\hline \multirow{3}{*}{$\begin{array}{c}{[5,6]-s p^{3}} \\
\text { (bent-extended) }\end{array}$} & chain/ $\mathrm{C}_{60}$ & -0.3 & 1.8 & 0.7 & $\begin{array}{c}-0.1 \\
\end{array}$ & -9.6 & 3.5 & 0.0 & $\begin{array}{l}-4.0 \\
\end{array}$ \\
\hline & isoall/ $\mathrm{C}_{60}$ & -0.4 & -0.3 & 0.6 & 0.0 & -13.7 & -2.2 & 0.0 & -16.1 \\
\hline & chain/isoall & 0.2 & 1.5 & 2.3 & 0.1 & -2.2 & -10.4 & -3.4 & -11.9 \\
\hline \multirow{3}{*}{$\begin{array}{c}{[5,6]-s p^{2}-} \\
\text { (bent-extended) }\end{array}$} & chain/ $\mathrm{C}_{60}$ & $\begin{array}{c}-0.3 \\
\end{array}$ & 1.3 & 0.7 & $\begin{array}{c}-0.1 \\
\end{array}$ & $\begin{array}{c}-11.0 \\
\end{array}$ & 3.6 & 0.0 & $\begin{array}{l}-5.8 \\
\end{array}$ \\
\hline & isoall/ $\mathrm{C}_{60}$ & -0.5 & 0.0 & 0.2 & -0.1 & -13.6 & -2.1 & 0.0 & -16.0 \\
\hline & chain/isoall & 0.2 & 1.5 & 1.3 & 0.1 & -1.7 & -7.8 & -3.3 & -9.9 \\
\hline \multirow{3}{*}{$\begin{array}{c}{[6,6]-s p^{3}-} \\
\text { (bent-extended) }\end{array}$} & chain $/ \mathrm{C}_{60}$ & 0.0 & 2.0 & 0.6 & 0.0 & -9.6 & 3.6 & 0.0 & -3.4 \\
\hline & isoall $/ \mathrm{C}_{60}$ & -0.2 & -0.1 & 0.6 & 0.1 & -14.5 & -2.4 & 0.0 & -16.6 \\
\hline & chain/isoall & 0.3 & 1.6 & 1.5 & 0.1 & -1.9 & -8.1 & -2.6 & -9.1 \\
\hline \multirow{3}{*}{$\begin{array}{c}{[6,6]-s p^{2}-} \\
\text { (bent-extended) }\end{array}$} & chain $/ \mathrm{C}_{60}$ & 年.1 & 1.1 & 1.2 & $\begin{array}{c}0.0 \\
\end{array}$ & -11.0 & 3.4 & 0.0 & $\begin{array}{l}-5.1 \\
\end{array}$ \\
\hline & isoall/ $\mathrm{C}_{60}$ & -0.2 & 0.0 & 0.5 & 0.0 & -14.3 & -2.1 & 0.0 & -16.2 \\
\hline & chain/isoall & 0.2 & 1.2 & 2.0 & 0.0 & -3.4 & -3.2 & 0.0 & -3.2 \\
\hline
\end{tabular}

Table S8. Bonding and nonbonding $\mathrm{FC}_{60}$ energy gain derived from fragment-pair summation of Table $\mathrm{S} 7$ for each of the four $\mathrm{FC}_{60}$ configurations studied. The fragment-gain total energy $\left(E_{\text {fragment-gain total }}\right)$ is contrasted to the total energy gain $\left(E_{M M \text {-gain total }}\right)$ obtained from Table S1. The difference between $E_{\text {fragment-gain total }}$ and $E_{M M \text {-gain total }}$ is presented in terms of percent deviation defined as $\left[\left(E_{\text {fragment-gain total }}-E_{M M \text {-gain total }}\right) / E_{M M \text {-gain total }}\right] * 100 \%$.

\begin{tabular}{|c|c|c|c|c|c|c|c|}
\hline \multirow{2}{*}{$\begin{array}{c}\mathrm{FC}_{60} \\
\text { configuration }\end{array}$} & \multirow{2}{*}{\begin{tabular}{|c|} 
Bonding \\
energy \\
$(\mathrm{kcal} / \mathrm{mol})$
\end{tabular}} & \multicolumn{3}{|c|}{$\begin{array}{c}\text { Nonbonding energy } \\
(\mathrm{kcal} / \mathrm{mol})\end{array}$} & \multirow{2}{*}{$\begin{array}{c}\boldsymbol{E}_{\text {fragment-gain total }} \\
(\mathrm{kcal} / \mathrm{mol})\end{array}$} & \multirow{2}{*}{$\begin{array}{c}\boldsymbol{E}_{\boldsymbol{M M} \text {-gain total }} \\
(\mathrm{kcal} / \mathrm{mol})\end{array}$} & \multirow{2}{*}{$\begin{array}{c}E_{\text {fragment-gain total }} \\
-E_{M M \text {-gain total }} \\
\% \text { Deviation }\end{array}$} \\
\hline & & $\mathrm{vdW}$ & Electrostatic & H-Bonds & & & \\
\hline $\begin{array}{l}{[5,6]-s p^{3}-\mathrm{FC}_{60}} \\
\text { (bent-extended) }\end{array}$ & 6.0 & -25.5 & -9.1 & -3.4 & -32.0 & -32.1 & $0.3 \%$ \\
\hline $\begin{array}{c}{[5,6]-s p^{2}-\mathrm{FC}_{60}} \\
\text { (bent-extended) }\end{array}$ & 4.3 & -26.3 & -6.3 & -3.3 & -31.6 & -31.7 & $0.3 \%$ \\
\hline $\begin{array}{l}{[6,6]-s p^{3}-\mathrm{FC}_{60}} \\
\text { (bent-extended) }\end{array}$ & 6.5 & -26.0 & -6.9 & -2.6 & -29.0 & -29.0 & $0.0 \%$ \\
\hline $\begin{array}{l}{[6,6]-s p^{2}-\mathrm{FC}_{60}} \\
\text { (bent-extended) }\end{array}$ & 6.1 & -28.7 & -1.9 & 0.0 & -24.5 & -24.5 & $0.0 \%$ \\
\hline
\end{tabular}

Helix-incorporated $\mathbf{F C}_{60}$ : As explained in the main text, quenched MD calculations were performed in order to understand the position where the $\mathrm{C}_{60}$ moiety of $\mathrm{FC}_{60}$ localizes upon bending within the $\mathrm{FC}_{12}$ helix wrapped around an $(8,6)-\mathrm{SWNT}$. Table S9 shows the energy contributions for the local minima obtained from the quench dynamic trajectories, that includes the entire $\delta_{1}$ FC12 helix and the $(8,6)-S W N T$.

Upon $\mathrm{FC}_{60}$ bending, the $\mathrm{C}_{60}$ moiety is localized on either the uracil or phenyl groove of the FC12 helix, as shown in Figure 6 of the main text and Figures S3 and S4 of the SI. Bending shortens the distance between the $\mathrm{C}_{60}$ and the isoalloxazine helix down to ca. $3.3 \AA$ (Table S10 and Figure S3). 
Table S9. Energy contributions for the extended and collapsed conformations of FC12-wrapped (8,6)-SWNTs (a), along with replacing one $\mathrm{FC} 12$ moiety with $[5,6]-s p^{3}-\mathrm{FC}_{60}$ (b) and $[6,6]-s p^{3}-\mathrm{FC}_{60}(\mathbf{c})$ in the extended and bent form, respectively. In terms of the bent conformations of $\mathrm{FC}_{60}$, the localization of the $\mathrm{C}_{60}$ moiety within the uracil and phenyl groove is identified, since it creates different local minima. Aside from $\mathrm{FC}_{60}$, these energy values also include the entire $\delta_{l}$ FC12 helix as well as the underlying $(8,6)$-SWNT.

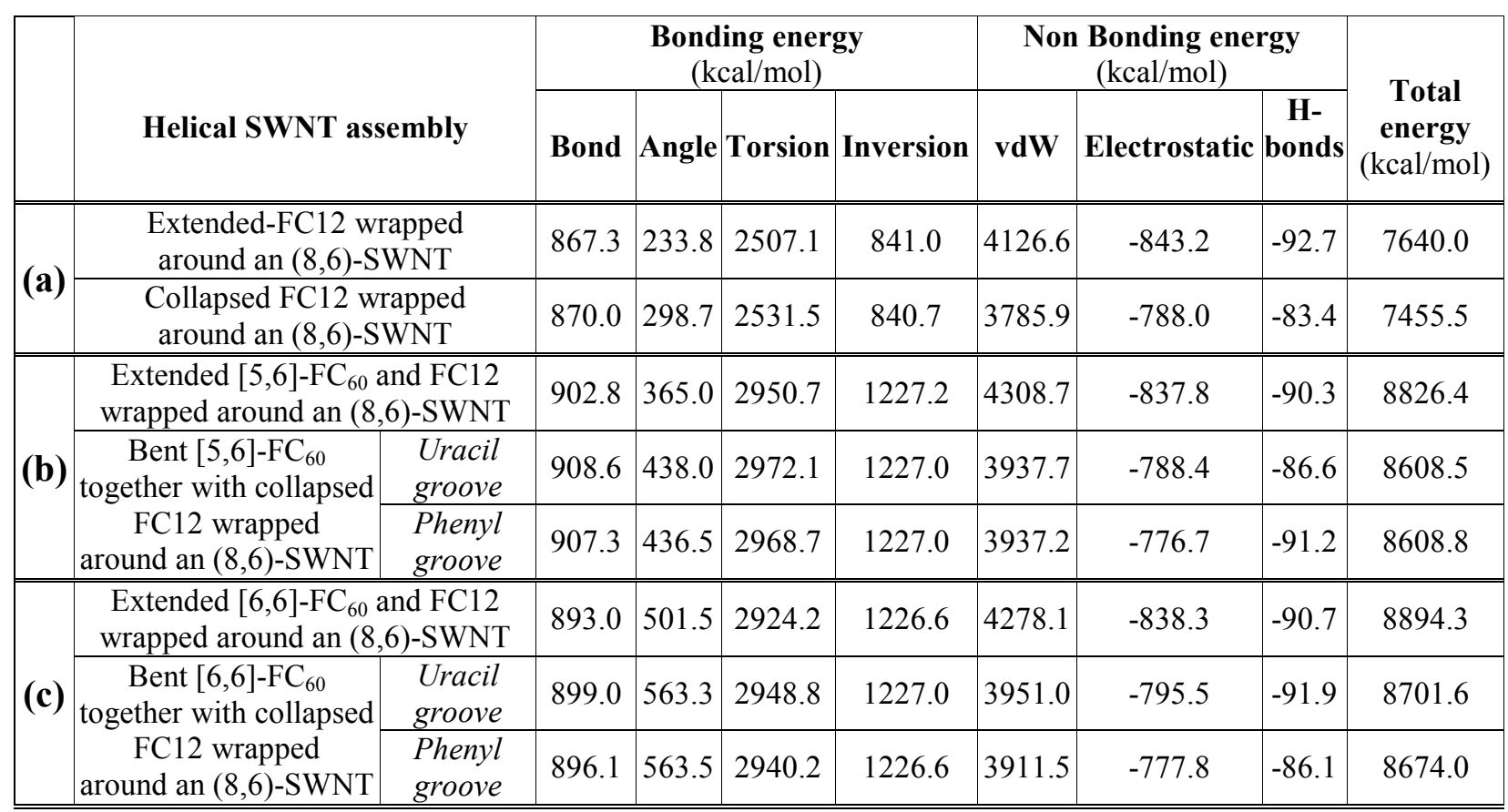
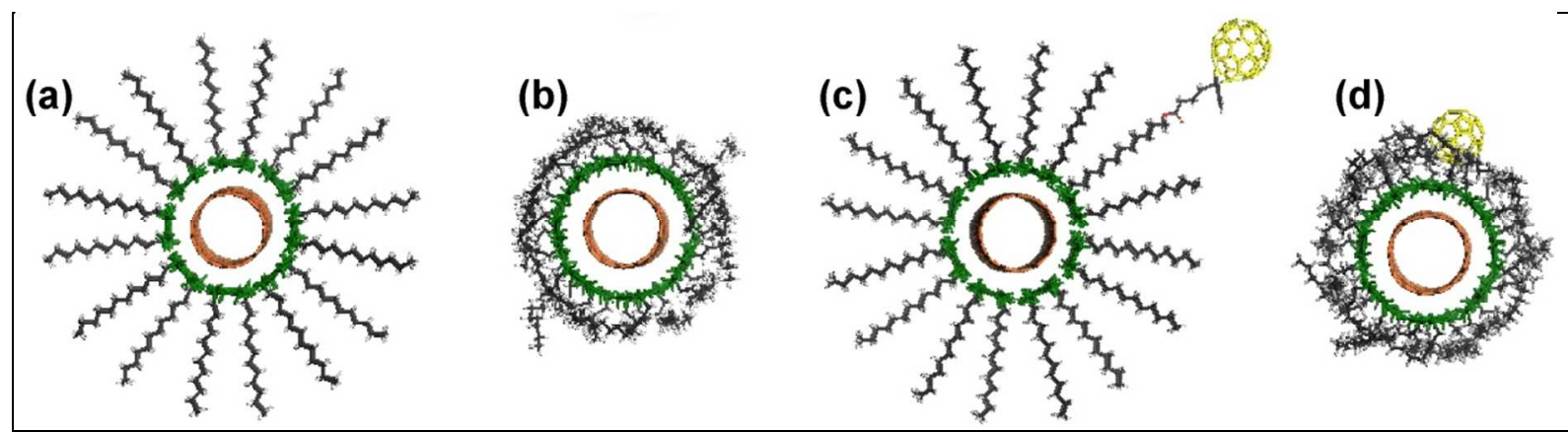

Figure S3. Molecular structure of an $8_{1}$ FC12 helix wrapped around an $(8,6)$-SWNT with the flexible C-12 chain in the extended (a) and collapsed (b) configurations. The helix is viewed along the long nanotube axis and is composed of two turns, each containing 16 FC12 molecules. In (c) and (d), 1 of the 32 FC12 units is replaced with a [5,6]-sp $-\mathrm{FC}_{60}$ in the extended and bent configurations, respectively. All isoalloxazine rings forming the $\delta_{1}$ helix are shown in green, while the $\mathrm{C}_{60}$ and the flexible chains are shown in yellow and gray, respectively.

Similar to the isolated $\mathrm{FC}_{60}$, the nonbonding contributions between the three fragments of $\mathrm{FC}_{60}$ (i.e. $\mathrm{C}_{60}$ and flexible chain and isoalloxazine ring) were also extracted. In order to perform this, the $\mathrm{C}-12$ flexible chains of the $\mathrm{FC} 12$ were removed to expose a $2 \times 3$ isoalloxazine patch, as shown in Figure S4. Table S11 shows the energy contributions (and differences shaded in gray) for the extended and bent conformations of $\mathrm{FC}_{60}$ onto the $2 \times 3$ isoalloxazine patch. Here it is important to stress that the reported energies for the $[5,6]-s p^{3}-$ and $[6,6]-s p^{3}-\mathrm{FC}_{60}$ configurations 
of Table S11 do not include contributions for the C-12 chains of the remaining 5 FC12s nor the underlying nanotube.

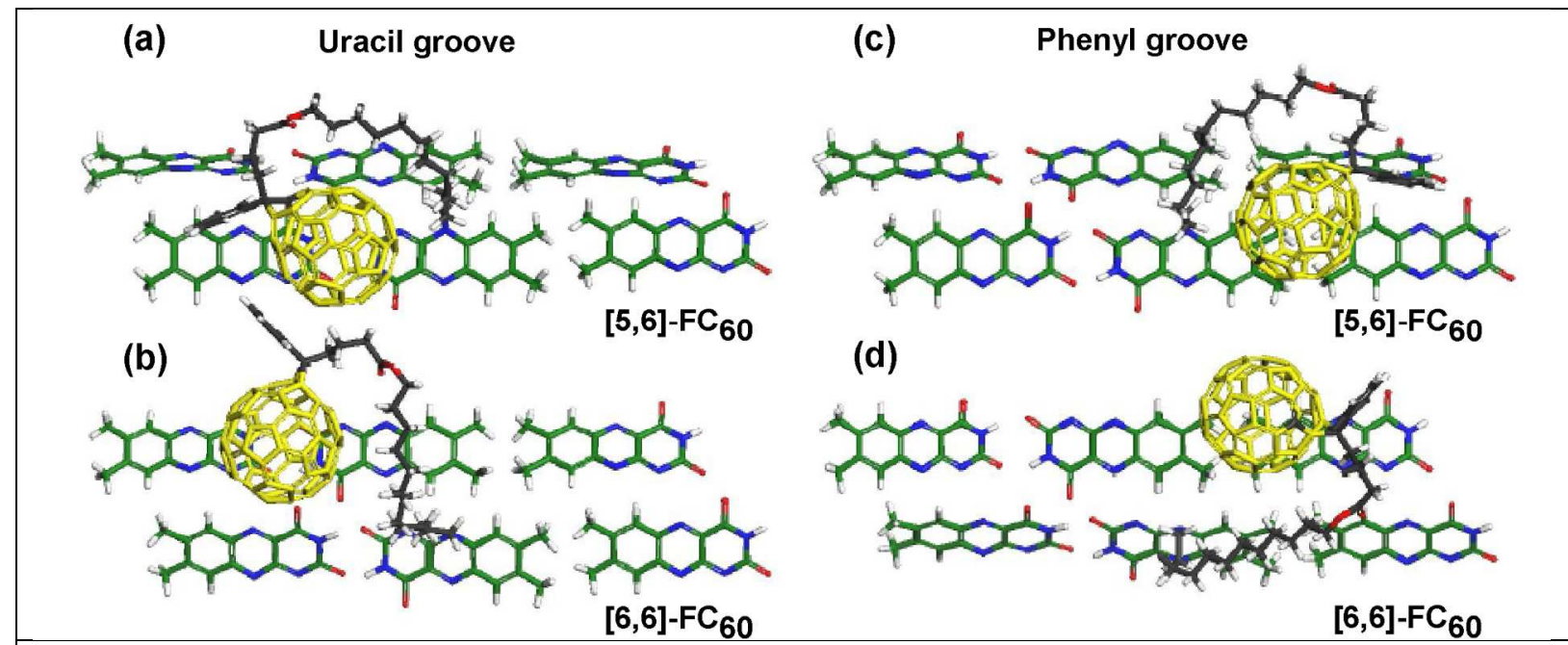

Figure S4. Minimized $\mathrm{FC}_{60}$ conformations onto helical $2 \times 3$ isoalloxazine patches. Here the $\mathrm{C}_{60}$ moiety is localized onto the uracil $(\mathbf{a}, \mathbf{b})$ and phenyl $(\mathbf{c}, \mathbf{d})$ grooves, for [5,6]- (top) and [6,6]-sp $-\mathrm{FC}_{60}$ (bottom), respectively. The $\mathrm{C}_{60}$ moiety is shown in yellow, while the carbons of the isoalloxazine ring are shown in green. The remaining $\mathrm{C}, \mathrm{H}, \mathrm{O}$ and $\mathrm{N}$ atoms are shown in gray, white, red and blue, respectively.

Subsequently, the pair contributions between the (a) flexible chain of $\mathrm{FC}_{60}$ and its $\mathrm{C}_{60}$ moiety; (b) $2 \times 3$ isoalloxazine patch and the $\mathrm{C}_{60}$; and (c) flexible chain and the $2 \times 3$ isoalloxazine patch, were calculated using eq. 1-5. Table S12 illustrates the individual fragment energies $\left(E_{f r a g}\right)$ obtained for $[5,6]-s p^{3}$ - and $[6,6]-s p^{3}-\mathrm{FC}_{60}$ configurations in uracil and phenyl grooves for both extended and bent conformations. As explained in eq. $1 E_{\text {frag, }}$ is composed of bonding and self-nonbonding terms. Similarly, Table S13 shows the energies of fragment-pairs (i.e. $E_{\text {frag } 1}+E_{\text {frag2 }}$, where the pairs are composed of the flexible chain with $\mathrm{C}_{60}, 2 \times 3$ isoalloxazine patch with $\mathrm{C}_{60}$, and flexible chain with $2 \times 3$ isoalloxazine patch) for all $\mathrm{FC}_{60}$ configurations. As described in eq. 2 , these fragment-pair energies include bonding as well as both self- and pairnonbonding contributions.

Using eq. 3 and the energy values of Tables S12 and S13, the pair interaction between two fragments, mostly comprising from $E_{\text {pair-nonbonding, were calculated and tabulated in Table S14. }}$ Table S15 depicts the recalculated fragment-pair energy $E_{(\text {frag } 1+f \text { frag } 2)}$ by employing eq. 4 . Table S16 presents the energy gain $\left(E_{\text {gain }}\right)$ calculated by eq. 5 for the three fragment-pairs with both $[5,6]-s p^{3}$ - and $[6,6]-s p^{3}-\mathrm{FC}_{60}$ configurations on either uracil or phenyl grooves of the isoalloxazine helix. The summation of all three fragment-pair energy contributions is shown in Table S17 with the sum of the three $E_{\text {gain }}$ terms shown as $E_{\text {fragment-gain total. }}$ The $E_{\text {fragment-gain total }}$ values are within $1 \%$ of the total energy gain $\left(E_{M M \text {-gain total }}\right)$ obtained from Table S11. This validates the aforementioned fragmentation treatment and allowed us to generate Table 2 in the main text, where the bonding, non-bonding (i.e. van der Waals (vdW), electrostatic (ES) and Hbond) and total energy values originate from Table S17.

These contributions are summarized in Table 2 of the main text where the sum of all bonding terms, vdW, ES, H-bonds and total energy values are extracted from Table S17. The fragmentation of the bonding energy contributions in Table 2 of the main text originates from the gray shaded cells of Table S12. In terms of vdW and ES terms, the breakdown in fragment-pair contributions stems from Table S16. 
Table S10. Average distance between the $\mathrm{C}_{60}$ moiety of a bent $[5,6]$ - and $[6,6]-s p^{3}-\mathrm{FC}_{60}$ from the uracil and phenyl groove of isoalloxazine helix wrapped around an $(8,6)$-SWNT. In the last column, the average distance of isoalloxazine helix and the $(8,6)$-SWNT is also listed.

\begin{tabular}{|c|c|c|c|}
\hline \multirow{2}{*}{ FC $_{\mathbf{6 0}}$ configuration } & Groove & $\begin{array}{c}\mathbf{C}_{\mathbf{6 0}} \text { /isoalloxazine } \\
\text { distance }(\AA)\end{array}$ & $\begin{array}{c}\text { Isoalloxazine/(8,6)-SWNT } \\
\text { distance }(\AA)\end{array}$ \\
\hline \multirow{2}{*}[5,6]{- sp $^{3}-\mathrm{FC}_{60}$} & Uracil & 3.3 & 3.4 \\
\cline { 2 - 4 } & Phenyl & 3.0 & 3.4 \\
\hline \multirow{2}{*}[6,6]{- sp $^{3}-\mathrm{FC}_{60}$} & Uracil & 3.4 & 3.4 \\
\cline { 2 - 4 } & Phenyl & 3.3 & 3.4 \\
\hline
\end{tabular}

Table S11. Energy contributions for the bent- and the extended-conformations of $\mathrm{FC}_{60}$, along with the net energy gain afforded via bending for each of the two $\mathrm{FC}_{60}$ configurations studied (i.e. $[5,6]-s p^{3}$ and $\left.[6,6]-\mathrm{sp}^{3}\right)$ on either the uracil or phenyl groove of the isoalloxazine helix. Here the energy values include only the $\mathrm{FC}_{60}$ and the $2 \times 3$ isoalloxazine patch devoid its five $\mathrm{C}-12$ chains.

\begin{tabular}{|c|c|c|c|c|c|c|c|c|c|}
\hline \multirow{2}{*}{$\begin{array}{c}\mathrm{FC}_{60} \\
\text { configuration }\end{array}$} & \multirow{2}{*}{ Groove } & \multicolumn{4}{|c|}{ Bonding Energy $(\mathrm{kcal} / \mathrm{mol})$} & \multicolumn{3}{|c|}{$\begin{array}{c}\text { Nonbonding Energy } \\
(\mathrm{kcal} / \mathrm{mol})\end{array}$} & \multirow{2}{*}{$\begin{array}{c}\boldsymbol{E}_{M M-g a i n}^{*} \text { tota } \\
(\mathrm{kcal} / \mathrm{mol})\end{array}$} \\
\hline & & Bond & Angle & Torsion & Inversion & VdW & Electrostatic & H-bonds & \\
\hline \multirow{2}{*}[5,6]{-bent } & uracil & 72.1 & 160.6 & 460.1 & 386.6 & 357.1 & -179.1 & -10.8 & 1246.6 \\
\hline & phenyl & 70.7 & 162.1 & 458.1 & 386.4 & 366.0 & -182.0 & -11.6 & 9.7 \\
\hline \multicolumn{2}{|c|}{$[5,6]$-extended } & 72.7 & 157.1 & 462.1 & 386.7 & 388.5 & -188.1 & -12.0 & 1267.0 \\
\hline \multirow{2}{*}{$\begin{array}{c}{[5,6]-} \\
\text { (bent-extended) }\end{array}$} & uracil & -0.6 & 3.5 & -2.0 & -0.1 & -31.4 & 9.0 & 1.2 & $\overline{-20.4}$ \\
\hline & phenyl & -2.0 & 5.0 & -4.0 & -0.3 & -22.5 & 6.1 & 0.4 & -17.3 \\
\hline \multirow{2}{*}[6,6]{-bent } & uracil & 63.0 & 293.8 & 432.0 & 386.0 & 338.8 & -177.2 & -13.1 & 1323.3 \\
\hline & phenyl & 62.0 & 293.5 & 431.0 & 386.1 & 343.6 & -179.2 & -10.9 & 1326.1 \\
\hline \multicolumn{2}{|c|}{$[6,6]$-extended } & 62.9 & 291.2 & 434.9 & 386.3 & 360.8 & -188.0 & -12.3 & 1335.8 \\
\hline \multirow{2}{*}{$\begin{array}{c}{[6,6]-} \\
\text { (bent-extended) }\end{array}$} & uracil & $\overline{0.1}$ & 2.6 & $\overline{-2.9}$ & -0.3 & -22.0 & 10.8 & 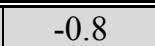 & -12.5 \\
\hline & phenyl & -0.9 & 2.3 & -3.9 & -0.2 & -17.2 & 8.8 & 1.4 & -9.7 \\
\hline
\end{tabular}

* The $E_{\text {total }}$ difference between the bent and extended $\mathrm{FC}_{60}$ conformation is herein defined as molecular mechanics (MM)-gain total $\left(\boldsymbol{E}_{M M \text {-gain total }}\right)$. 
Table S12. Energy contributions for the three $\mathrm{FC}_{60}$ fragments (i.e. flexible chain, $\mathrm{C}_{60}$ and $2 \times 3$ isoalloxazine patch) of the extended- and bent-conformations (on either uracil or phenyl grooves of the isoalloxazine helix) for $[5,6]-s p^{3}$ and $[6,6]-\mathrm{sp}^{3}-\mathrm{FC}_{60}$ configurations. As described in eq. 1, these fragment energies are composed of bonding and selfnonbonding terms.

\begin{tabular}{|c|c|c|c|c|c|c|c|c|c|}
\hline \multirow[t]{2}{*}{$F_{60}$ configuration } & \multirow[t]{2}{*}{ Fragment } & \multicolumn{4}{|c|}{ Bonding energy (kcal/mol) } & \multicolumn{3}{|c|}{$\begin{array}{c}\text { Self-nonbonding energy } \\
(\mathrm{kcal} / \mathrm{mol})\end{array}$} & \multirow{2}{*}{$\begin{array}{c}\boldsymbol{E}_{\text {frag }} \\
(\mathrm{kcal} / \mathrm{mol})\end{array}$} \\
\hline & & Bond & Angle & Torsion & Inversion & vdW & Electrostatic & H-bonds & \\
\hline \multirow{3}{*}[5,6]{-uracil-bent } & chain & 3.3 & 6.1 & 2.3 & 0.0 & 19.9 & 9.3 & 0.0 & 41.0 \\
\hline & $\mathrm{C}_{60}$ & 33.4 & 131.9 & 444.1 & 386.0 & 171.0 & 1.9 & 0.0 & 1168.3 \\
\hline & $2 \times 3$ isoall & 33.6 & 19.8 & 13.8 & 0.5 & 187.8 & -189.9 & -10.8 & 54.9 \\
\hline \multirow{3}{*}[5,6]{-phenyl-bent } & chain & 3.0 & 5.4 & 1.5 & 0.0 & 21.9 & 9.3 & 0.0 & 41.1 \\
\hline & $\mathrm{C}_{60}$ & 34.7 & 132.3 & 443.8 & 385.9 & 169.6 & 3.2 & 0.0 & 1169.5 \\
\hline & $2 \times 3$ isoall & 31.3 & 21.7 & 13.0 & 0.5 & 195.2 & -191.8 & -11.6 & 58.3 \\
\hline \multirow{3}{*}[5,6]{-extended } & chain & 2.8 & 3.8 & 0.4 & 0.0 & 21.7 & 9.5 & 0.0 & 38.2 \\
\hline & $\mathrm{C}_{60}$ & 35.0 & 132.0 & 444.0 & 385.9 & 169.4 & 1.7 & 0.0 & 1168.0 \\
\hline & $2 \times 3$ isoall & 33.0 & 18.9 & 17.7 & 0.8 & 191.1 & -196.3 & -12.0 & 53.2 \\
\hline \multirow{3}{*}{$\begin{array}{l}{[5,6] \text {-uracil-(bent- }} \\
\text { extended) }\end{array}$} & chain & 0.5 & 2.3 & 1.9 & 0.0 & \begin{tabular}{|l|}
-1.7 \\
\end{tabular} & -0.2 & 0.0 & 2.8 \\
\hline & $\mathrm{C}_{60}$ & -1.6 & -0.1 & 0.1 & 0.1 & 1.5 & 0.2 & 0.0 & 0.2 \\
\hline & $2 \times 3$ isoall & 0.7 & 0.9 & -3.9 & -0.3 & -3.2 & 6.4 & 1.2 & 1.7 \\
\hline \multirow{3}{*}{$\begin{array}{c}{[5,6]-\text { phenyl-(bent- }} \\
\text { extended) }\end{array}$} & chain & 0.2 & 1.6 & 1.1 & 0.0 & 0.2 & -0.2 & 0.0 & 2.9 \\
\hline & $\mathrm{C}_{60}$ & -0.3 & 0.4 & -0.2 & 0.0 & 0.1 & 1.5 & 0.0 & 1.4 \\
\hline & $2 \times 3$ isoall & -1.6 & 2.7 & -4.7 & -0.3 & 4.1 & 4.5 & 0.4 & 5.1 \\
\hline \multirow{3}{*}{ [6,6]-uracil-bent } & chain & 2.9 & 4.8 & 2.2 & 0.0 & 20.6 & 9.4 & 0.0 & 39.9 \\
\hline & $\mathrm{C}_{60}$ & 24.9 & 264.0 & 416.3 & 385.5 & 147.5 & 1.0 & 0.0 & 1239.2 \\
\hline & $2 \times 3$ isoall & 33.9 & 20.7 & 13.1 & 0.5 & 191.7 & -183.5 & -13.1 & 63.4 \\
\hline \multirow{3}{*}[6,6]{-phenyl-bent } & chain & 3.0 & $\begin{array}{c}5.6 \\
\end{array}$ & 2.2 .2 & 0.0 & 20.4 & 7.1 & 0.0 & $\begin{array}{l}38.3 \\
\end{array}$ \\
\hline & $\mathrm{C}_{60}$ & 25.2 & 263.8 & 416.3 & 385.5 & 147.3 & 2.6 & 0.0 & 1240.7 \\
\hline & $2 \times 3$ isoall & 32.7 & 19.7 & 11.9 & 0.6 & 190.9 & -188.5 & -10.9 & 56.4 \\
\hline \multirow{3}{*}[6,6]{-extended } & chain & 2.8 & 4.3 & 0.3 & 0.0 & 20.5 & 10.1 & 0.0 & 38.0 \\
\hline & $\mathrm{C}_{60}$ & 25.7 & 263.8 & 416.3 & 385.5 & 146.7 & 1.5 & 0.0 & 1239.4 \\
\hline & $2 \times 3$ isoall & 33.1 & 19.1 & 17.6 & 0.8 & 191.6 & -196.1 & -12.3 & 53.8 \\
\hline \multirow{3}{*}{$\begin{array}{l}{[6,6] \text {-uracil-(bent- }} \\
\text { extended) }\end{array}$} & chain & 0.2 & 1.0 & 1.1 & 0.0 & 0.5 & -0.4 & 0.0 & 2.5 \\
\hline & $\mathrm{C}_{60}$ & $\begin{array}{ll}-0.8 \\
\end{array}$ & 0.3 & 0.0 & 0.0 & 1.1 & -1.3 & 0.0 & -0.7 \\
\hline & $2 \times 3$ isoall & 0.8 & 1.7 & -4.5 & -0.3 & 0.2 & 12.6 & -0.8 & 9.6 \\
\hline \multirow{3}{*}{$\begin{array}{c}{[6,6] \text {-phenyl-(bent- }} \\
\text { extended) }\end{array}$} & chain & 0.3 & 1.6 & 1.8 & 0.0 & 0.4 & -3.0 & 0.0 & 1.1 \\
\hline & $\mathrm{C}_{60}$ & -0.5 & 0.2 & -0.1 & 0.0 & 0.7 & 0.2 & 0.0 & 0.5 \\
\hline & $2 \times 3$ isoall & -0.4 & 0.7 & -5.8 & -0.2 & -0.6 & 7.6 & 1.4 & 2.6 \\
\hline
\end{tabular}


Table S13. Energy contributions for the three $\mathrm{FC}_{60}$ fragment-pairs (i.e. flexible chain with $\mathrm{C}_{60}, 2 \times 3$ isoalloxazine patch with $\mathrm{C}_{60}$, and flexible chain with $2 \times 3$ isoalloxazine patch) of the extended- and bent-conformations (on either uracil or phenyl grooves of the isoalloxazine helix) for $[5,6]-s p^{3}$ - and $[6,6]-\mathrm{s}^{3}-\mathrm{FC}_{60}$ configurations. As described in eq. 2, these fragment-pair energies are composed of bonding and nonbonding terms, the latter of which comprises from both self- and pair-nonbonding contributions.

\begin{tabular}{|c|c|c|c|c|c|c|c|c|c|}
\hline \multirow{2}{*}{$\begin{array}{c}\mathrm{FC}_{60} \\
\text { configuration }\end{array}$} & \multirow{2}{*}{ Fragment-pair } & \multicolumn{4}{|c|}{ Bonding energy $(\mathrm{kcal} / \mathrm{mol})$} & \multicolumn{3}{|c|}{\begin{tabular}{|c|} 
Self-nonbonding and pair- \\
nonbonding energy $(\mathrm{kcal} / \mathrm{mol})$
\end{tabular}} & \multirow{2}{*}{$\begin{array}{c}\boldsymbol{E}_{\text {fragl }}+\text { frag } 2 \\
(\mathrm{kcal} / \mathrm{mol})\end{array}$} \\
\hline & & Bond & Angle & Torsion & Inversion & vdW & Electrostatic & H-bonds & \\
\hline \multirow{3}{*}{$\begin{array}{l}{[5,6] \text {-uracil- }} \\
\text { bent }\end{array}$} & chain $/ \mathrm{C}_{60}$ & 37.8 & 138.9 & 446.5 & 386.0 & 184.5 & 10.8 & 0.0 & 1204.6 \\
\hline & $2 \times 3$ isoall $/ \mathrm{C}_{60}$ & 67.0 & 151.7 & 457.9 & 386.5 & 342.0 & -186.8 & -10.8 & 1207.6 \\
\hline & chain $/ 2 \times 3$ isoall & 37.6 & 27.7 & 15.9 & 0.6 & 209.4 & -181.9 & -10.8 & 98.5 \\
\hline \multirow{3}{*}{$\begin{array}{l}\text { [5,6]-phenyl- } \\
\text { bent }\end{array}$} & chain $/ \mathrm{C}_{60}$ & 38.8 & 138.9 & 445.4 & 385.9 & 184.7 & 11.9 & 0.0 & 1205.7 \\
\hline & $2 \times 3$ isoall $/ \mathrm{C}_{60}$ & 66.0 & 154.0 & 456.8 & 386.4 & 349.3 & -187.9 & -11.6 & 1213.1 \\
\hline & chain $/ 2 \times 3$ isoall & 34.9 & 28.6 & 14.2 & 0.5 & 218.6 & -185.4 & -11.6 & 99.9 \\
\hline \multirow{3}{*}[5,6]{-extended } & chain/ $\mathrm{C}_{60}$ & 38.8 & 137.0 & 444.5 & 385.9 & 194.6 & 11.8 & 0.0 & 1212.6 \\
\hline & $2 \times 3$ isoall $/ \mathrm{C}_{60}$ & 67.9 & 150.9 & 461.7 & 386.7 & 360.5 & -194.6 & -12.0 & 1221.2 \\
\hline & chain $/ 2 \times 3$ isoall & 36.6 & 24.0 & 18.0 & 0.8 & 215.6 & -190.3 & -12.0 & 92.6 \\
\hline \multirow{3}{*}{$\begin{array}{l}{[6,6]-\text { uracil- }} \\
\text { bent }\end{array}$} & chain/ $\mathrm{C}_{60}$ & 28.1 & 271.7 & 419.2 & 385.5 & 158.7 & 10.4 & 0.0 & 1273.6 \\
\hline & $2 \times 3$ isoall $/ \mathrm{C}_{60}$ & 58.8 & 284.7 & 429.4 & 386.0 & 325.1 & -182.8 & -13.1 & 1288.1 \\
\hline & chain $/ 2 \times 3$ isoall & 37.8 & 26.9 & 15.0 & 0.5 & 214.9 & -178.0 & -13.1 & 104.1 \\
\hline \multirow{3}{*}{$\begin{array}{c}{[6,6]-\text { phenyl- }} \\
\text { bent }\end{array}$} & chain/ $\mathrm{C}_{60}$ & 28.5 & 272.3 & 419.3 & 385.5 & 162.0 & 9.9 & 0.0 & 1277.5 \\
\hline & $2 \times 3$ isoall $/ \mathrm{C}_{60}$ & 57.9 & 283.5 & 428.2 & 386.1 & 328.1 & -184.2 & -10.9 & 1288.7 \\
\hline & chain $/ 2 \times 3$ isoall & 36.5 & 26.7 & 13.9 & 0.6 & 212.2 & -183.7 & -10.9 & 95.3 \\
\hline \multirow{3}{*}[6,6]{-extended } & chain/ $\mathrm{C}_{60}$ & 28.8 & 271.0 & 417.5 & 385.5 & 166.4 & 12.3 & 0.0 & 1281.4 \\
\hline & $2 \times 3$ isoall $/ \mathrm{C}_{60}$ & 58.8 & 282.8 & 434.0 & 386.3 & 338.2 & -194.6 & -12.3 & 1293.2 \\
\hline & chain $/ 2 \times 3$ isoall & 36.9 & 24.4 & 17.7 & 0.8 & 214.9 & -190.1 & -12.3 & 92.4 \\
\hline
\end{tabular}


Table S14. Energy contributions of the pair-interactions (as obtained from Tables S12, S13 and eq. 3) for the three fragment-pairs (i.e. flexible chain with $\mathrm{C}_{60}, 2 \times 3$ isoalloxazine patch with $\mathrm{C}_{60}$, and flexible chain with $2 \times 3$ isoalloxazine patch) of the extended- and bent-conformations (on either uracil or phenyl grooves of the isoalloxazine helix) for $[5,6]-s p^{3}$ - and $[6,6]-\mathrm{sp}^{3}-\mathrm{FC}_{60}$ configurations. The non-zero bonding energy terms for two of the three pairs (i.e. flexible chain/ $\mathrm{C}_{60}$ and flexible chain/isoalloxazine) originate from bond formation between these two fragments.

\begin{tabular}{|c|c|c|c|c|c|c|c|c|c|}
\hline \multirow{2}{*}{$\begin{array}{c}\mathrm{FC}_{60} \\
\text { configuration }\end{array}$} & \multirow{2}{*}{ Fragment-pair } & \multicolumn{4}{|c|}{ Bonding energy $(\mathrm{kcal} / \mathrm{mol})$} & \multicolumn{3}{|c|}{$\begin{array}{c}\text { Pair-nonbonding energy } \\
(\mathrm{kcal} / \mathrm{mol})\end{array}$} & \multirow{2}{*}{$\begin{array}{c}\boldsymbol{E}_{\text {pair interaction }} \\
(\mathrm{kcal} / \mathrm{mol})\end{array}$} \\
\hline & & Bond & Angle & Torsion & Inversion & vdW & Electrostatic & H-bonds & \\
\hline \multirow{3}{*}{$\begin{array}{c}{[5,6]-\text { uracil- }} \\
\text { bent }\end{array}$} & chain/ $\mathrm{C}_{60}$ & 1.1 & 0.9 & 0.1 & 0.0 & -6.3 & -0.4 & 0.0 & -4.7 \\
\hline & $2 \times 3$ isoall $/ \mathrm{C}_{60}$ & 0.0 & 0.0 & 0.0 & 0.0 & -16.8 & 1.2 & 0.0 & -15.6 \\
\hline & chain $/ 2 \times 3$ isoall & 0.6 & 1.8 & -0.2 & 0.0 & 1.6 & -1.3 & 0.0 & 2.6 \\
\hline \multirow{3}{*}{$\begin{array}{l}{[5,6]-\text { phenyl- }} \\
\text { bent }\end{array}$} & chain/ $\mathrm{C}_{60}$ & 1.1 & 1.2 & 0.1 & 0.0 & -6.7 & -0.6 & 0.0 & -4.9 \\
\hline & $2 \times 3$ isoall $/ \mathrm{C}_{60}$ & 0.0 & 0.0 & 0.0 & 0.0 & -15.5 & 0.8 & 0.0 & -14.7 \\
\hline & chain $/ 2 \times 3$ isoall & 0.6 & 1.5 & -0.3 & 0.0 & 1.6 & -2.9 & 0.0 & 0.5 \\
\hline \multirow{3}{*}[5,6]{-extended } & chain/ $\mathrm{C}_{60}$ & 1.1 & 1.2 & 0.1 & 0.0 & 3.5 & 0.6 & 0.0 & 6.4 \\
\hline & $2 \times 3$ isoall $/ \mathrm{C}_{60}$ & 0.0 & 0.0 & 0.0 & 0.0 & 0.0 & 0.0 & 0.0 & 0.0 \\
\hline & chain $/ 2 \times 3$ isoall & 0.9 & 1.2 & -0.1 & 0.0 & 2.8 & -3.6 & 0.0 & 1.2 \\
\hline \multirow{3}{*}{$\begin{array}{c}{[6,6]-\text { uracil- }} \\
\text { bent }\end{array}$} & chain/ $\mathrm{C}_{60}$ & 0.3 & 2.9 & 0.7 & 0.0 & -9.4 & 0.0 & 0.0 & -5.5 \\
\hline & $2 \times 3$ isoall $/ \mathrm{C}_{60}$ & 0.0 & 0.0 & 0.0 & 0.0 & -14.2 & -0.3 & 0.0 & -14.5 \\
\hline & chain $/ 2 \times 3$ isoall & 0.9 & 1.4 & -0.3 & 0.0 & 2.6 & -3.8 & 0.0 & 0.7 \\
\hline \multirow{3}{*}{$\begin{array}{c}{[6,6]-p h e n y l-} \\
\text { bent }\end{array}$} & chain $/ \mathrm{C}_{60}$ & 0.3 & 3.0 & 0.8 & 0.0 & -5.7 & 0.2 & 0.0 & -1.4 \\
\hline & $2 \times 3$ isoall $/ \mathrm{C}_{60}$ & 0.0 & 0.0 & 0.0 & 0.0 & -10.2 & 1.8 & 0.0 & -8.4 \\
\hline & chain $/ 2 \times 3$ isoall & 0.7 & 1.4 & -0.1 & 0.0 & 0.9 & -2.3 & 0.0 & 0.6 \\
\hline \multirow{3}{*}[6,6]{-extended } & chain/ $\mathrm{C}_{60}$ & 0.3 & 3.0 & 0.9 & 0.0 & -0.8 & 0.6 & 0.0 & 4.0 \\
\hline & $2 \times 3$ isoall $/ \mathrm{C}_{60}$ & 0.0 & 0.0 & 0.0 & 0.0 & 0.0 & 0.0 & 0.0 & 0.0 \\
\hline & chain $/ 2 \times 3$ isoall & 1.0 & 1.1 & -0.2 & 0.0 & 2.9 & -4.1 & 0.0 & 0.6 \\
\hline
\end{tabular}


Table S15. Recalculated energy contributions (according to eq. 4) of the three $\mathrm{FC}_{60}$ fragment-pairs (i.e. flexible chain with $\mathrm{C}_{60}, 2 \times 3$ isoalloxazine patch with $\mathrm{C}_{60}$, and flexible chain with $2 \times 3$ isoalloxazine patch) of extended- and bent-conformations (on either uracil or phenyl grooves of the isoalloxazine helix) for $[5,6]-s p^{3}-$ and $[6,6]-\mathrm{sp}^{3}-\mathrm{FC}_{60}$ configurations. The recalculated fragment-pair energies account for double counting of both $E_{\text {bonding }}$ and $E_{\text {self- }}$ nonbonding terms.

\begin{tabular}{|c|c|c|c|c|c|c|c|c|c|}
\hline \multirow{2}{*}{$\begin{array}{c}\mathbf{F C}_{60} \\
\text { configuration }\end{array}$} & \multirow{2}{*}{ Fragment-pair } & \multicolumn{4}{|c|}{ Bonding energy $(\mathrm{kcal} / \mathrm{mol})$} & \multicolumn{3}{|c|}{\begin{tabular}{|c|} 
Self-nonbonding and pair- \\
nonbonding energy $(\mathrm{kcal} / \mathrm{mol})$
\end{tabular}} & \multirow{2}{*}{$\begin{array}{l}\boldsymbol{E}_{\text {fragl+frag }}^{\prime} \\
(\mathrm{kcal} / \mathrm{mol})\end{array}$} \\
\hline & & Bond & Angle & Torsion & Inversion & vdW & Electrostatic & H-bonds & \\
\hline \multirow{3}{*}{$\begin{array}{c}{[5,6] \text {-uracil- }} \\
\text { bent }\end{array}$} & chain $/ \mathrm{C}_{60}$ & 19.4 & 69.9 & 223.3 & 193.0 & 89.1 & 5.2 & 0.0 & 599.9 \\
\hline & $2 \times 3$ isoall $/ \mathrm{C}_{60}$ & 33.5 & 75.9 & 229.0 & 193.3 & 162.6 & -92.8 & -5.4 & 596.0 \\
\hline & chain $/ 2 \times 3$ isoall & 19.1 & 14.8 & 7.9 & 0.3 & 105.5 & -91.6 & -5.4 & 50.6 \\
\hline \multirow{3}{*}{$\begin{array}{l}{[5,6]-\text { phenyl- }} \\
\text { bent }\end{array}$} & chain/ $\mathrm{C}_{60}$ & 20.0 & 70.0 & 222.8 & 193.0 & 89.0 & 5.7 & 0.0 & 600.4 \\
\hline & $2 \times 3$ isoall $/ \mathrm{C}_{60}$ & 33.0 & 77.0 & 228.4 & 193.2 & 166.9 & -93.5 & -5.8 & 599.2 \\
\hline & chain $/ 2 \times 3$ isoall & 17.8 & 15.0 & 6.9 & 0.3 & 110.1 & -94.1 & -5.8 & 50.2 \\
\hline \multirow{3}{*}[5,6]{-extended } & chain $/ \mathrm{C}_{60}$ & 20.0 & 69.1 & 222.3 & 193.0 & 99.0 & 6.2 & 0.0 & 609.5 \\
\hline & $2 \times 3$ isoall $/ \mathrm{C}_{60}$ & 34.0 & 75.5 & 230.9 & 193.3 & 180.3 & -97.3 & -6.0 & 610.6 \\
\hline & chain $/ 2 \times 3$ isoall & 18.7 & 12.6 & 8.9 & 0.4 & 109.2 & -97.0 & -6.0 & 46.9 \\
\hline \multirow{3}{*}{$\begin{array}{c}{[6,6] \text {-uracil- }} \\
\text { bent }\end{array}$} & chain/ $\mathrm{C}_{60}$ & 14.2 & 137.3 & 210.0 & 192.7 & 74.6 & 5.2 & 0.0 & 634.1 \\
\hline & $2 \times 3$ isoall $/ \mathrm{C}_{60}$ & 29.4 & 142.4 & 214.7 & 193.0 & 155.4 & -91.5 & -6.5 & 636.8 \\
\hline & chain $/ 2 \times 3$ isoall & 19.3 & 14.2 & 7.3 & 0.2 & 108.7 & -90.9 & -6.5 & 52.4 \\
\hline \multirow{3}{*}{$\begin{array}{c}{[6,6]-\text { phenyl- }} \\
\text { bent }\end{array}$} & chain/ $\mathrm{C}_{60}$ & 14.4 & 137.7 & 210.0 & 192.8 & 78.1 & 5.0 & 0.0 & 638.0 \\
\hline & $2 \times 3$ isoall $/ \mathrm{C}_{60}$ & 29.0 & 141.7 & 214.1 & 193.1 & 158.9 & -91.2 & -5.4 & 640.1 \\
\hline & chain $/ 2 \times 3$ isoall & 18.6 & 14.1 & 6.9 & 0.3 & 106.5 & -93.0 & -5.4 & 48.0 \\
\hline \multirow{3}{*}[6,6]{-extended } & chain $/ \mathrm{C}_{60}$ & 14.6 & 137.0 & 209.2 & 192.8 & 82.8 & 6.4 & 0.0 & 642.7 \\
\hline & $2 \times 3$ isoall $/ \mathrm{C}_{60}$ & 29.4 & 141.4 & 217.0 & 193.1 & 169.1 & -97.3 & -6.1 & 646.6 \\
\hline & chain $/ 2 \times 3$ isoall & 18.9 & 12.8 & 8.7 & 0.4 & 108.9 & -97.1 & -6.1 & 46.5 \\
\hline
\end{tabular}


Table S16. Calculated energy gain from bending (according to eq. 5) for the three $\mathrm{FC}_{60}$ fragment-pairs (i.e. flexible chain with $\mathrm{C}_{60}, 2 \times 3$ isoalloxazine patch with $\mathrm{C}_{60}$, and flexible chain with $2 \times 3$ isoalloxazine patch) on either uracil or phenyl grooves of the isoalloxazine helix for $[5,6]-s p^{3}$ - and $[6,6]-s p^{3}-\mathrm{FC}_{60}$ configurations.

\begin{tabular}{|c|c|c|c|c|c|c|c|c|c|}
\hline \multirow{2}{*}{$\underset{\text { configuration }}{\mathbf{F C}_{60}}$} & \multirow{2}{*}{ Fragment-pair } & \multicolumn{4}{|c|}{ Bonding energy $(\mathrm{kcal} / \mathrm{mol})$} & \multicolumn{3}{|c|}{$\begin{array}{c}\text { Nonbonding energy } \\
(\mathrm{kcal} / \mathrm{mol})\end{array}$} & \multirow{2}{*}{$\begin{array}{c}\boldsymbol{E}_{\text {gain }} \\
(\mathrm{kcal} / \mathrm{mol})\end{array}$} \\
\hline & & Bond & Angle & Torsion & Inversion & vdW & Electrostatic & H-bonds & \\
\hline \multirow{3}{*}{$\begin{array}{c}{[5,6] \text {-uracil }} \\
\text { (bent-extended) }\end{array}$} & chain/ $\mathrm{C}_{60}$ & -0.5 & 0.8 & 1.0 & 0.0 & -9.9 & -1.0 & 0.0 & -9.6 \\
\hline & $2 \times 3$ isoall/ $\mathrm{C}_{60}$ & -0.5 & 0.4 & -1.9 & -0.1 & -17.7 & 4.6 & 0.6 & -14.6 \\
\hline & chain $/ 2 \times 3$ isoall & 0.4 & 2.2 & -1.0 & -0.1 & -3.7 & 5.4 & 0.6 & 3.7 \\
\hline \multirow{3}{*}{$\begin{array}{c}{[5,6]-\text { phenyl }} \\
\text { (bent-extended) }\end{array}$} & chain $/ \mathrm{C}_{60}$ & 0.0 & 1.0 & 0.5 & 0.0 & -10.0 & -0.6 & 0.0 & -9.1 \\
\hline & $2 \times 3$ isoall $/ \mathrm{C}_{60}$ & -1.0 & 1.5 & -2.5 & -0.1 & -13.4 & 3.8 & 0.2 & -11.4 \\
\hline & chain $/ 2 \times 3$ isoall & -1.0 & 2.4 & -2.0 & -0.1 & 0.9 & 2.8 & 0.2 & 3.3 \\
\hline \multirow{3}{*}{$\begin{array}{c}{[6,6] \text {-uracil }} \\
\text { (bent-extended) }\end{array}$} & chain/ $\mathrm{C}_{60}$ & -0.4 & 0.3 & 0.8 & 0.0 & -8.1 & -1.2 & 0.0 & -8.6 \\
\hline & $2 \times 3$ isoall $/ \mathrm{C}_{60}$ & 0.0 & 1.0 & -2.3 & -0.2 & -13.7 & 5.8 & -0.4 & -9.8 \\
\hline & chain $/ 2 \times 3$ isoall & 0.4 & 1.4 & -1.4 & -0.1 & -0.2 & 6.2 & -0.4 & 5.9 \\
\hline \multirow{3}{*}{$\begin{array}{c}{[6,6]-\text { phenyl }} \\
\text { (bent-extended) }\end{array}$} & chain/ $\mathrm{C}_{60}$ & -0.1 & 0.7 & 0.8 & 0.0 & -4.7 & -1.4 & 0.0 & -4.7 \\
\hline & $2 \times 3$ isoall $/ \mathrm{C}_{60}$ & -0.4 & 0.3 & -2.9 & -0.1 & -10.2 & 6.1 & 0.7 & -6.5 \\
\hline & chain $/ 2 \times 3$ isoall & -0.4 & 1.3 & -1.8 & -0.1 & -2.4 & 4.1 & 0.7 & 1.4 \\
\hline
\end{tabular}

Table S17. Bonding and nonbonding energy gain derived from fragment-pair summation of Table $\mathrm{S} 16$ for each of the two $\mathrm{FC}_{60}$ configurations (i.e. $[5,6]-s p^{3}$ and $[6,6]-\mathrm{s} p^{3}$ ) bending on either the uracil or the phenyl groove of the isoalloxazine helix. The fragment-gain total energy $\left(E_{\text {fragment-gain total }}\right)$ is contrasted to the total energy gain $\left(E_{M M \text {-gain total }}\right)$ obtained from Table S11. The difference between $E_{\text {fragment-gain total }}$ and $E_{M M \text {-gain total }}$ is presented in terms of percent deviation defined as $\left[\left(E_{\text {fragment-gain total }}-E_{M M \text {-gain total }}\right) / E_{M M \text {-gain total }}\right] * 100 \%$.

\begin{tabular}{|c|c|c|c|c|c|c|c|c|}
\hline \multirow{2}{*}{$\begin{array}{c}\mathrm{FC}_{60} \\
\text { configuration }\end{array}$} & \multirow{2}{*}{ Groove } & \multirow{2}{*}{$\begin{array}{c}\text { Bonding } \\
\text { energy } \\
(\mathrm{kcal} / \mathrm{mol})\end{array}$} & \multicolumn{3}{|c|}{$\begin{array}{l}\text { Nonbonding energy } \\
(\mathrm{kcal} / \mathrm{mol})\end{array}$} & \multirow{2}{*}{$\begin{array}{c}\boldsymbol{E}_{\text {fragment-gain total }} \\
(\mathrm{kcal} / \mathrm{mol})\end{array}$} & \multirow{2}{*}{$\begin{array}{c}E_{M M \text {-gain total }} \\
(\mathrm{kcal} / \mathrm{mol})\end{array}$} & \multirow{2}{*}{$\begin{array}{c}E_{\text {fragment-gain total }} \\
-E_{M M-\text { gain total }} \\
\% \text { Deviation }\end{array}$} \\
\hline & & & vdW & Electrostatic & $\begin{array}{c}\text { H- } \\
\text { Bonds }\end{array}$ & & & \\
\hline \multirow{2}{*}{$\begin{array}{c}5,6] \\
\text { (bent-extended) }\end{array}$} & uracil & 0.7 & -31.3 & 8.9 & 1.2 & -20.5 & -20.4 & $0.5 \%$ \\
\hline & phenyl & -1.3 & -22.5 & 6.0 & 0.4 & -17.4 & -17.3 & $0.6 \%$ \\
\hline \multirow{2}{*}{$\begin{array}{c}{[6,6]} \\
\text { (bent-extended) }\end{array}$} & uracil & -0.5 & -22.0 & 10.8 & -0.8 & -12.5 & -12.5 & $0.0 \%$ \\
\hline & phenyl & -2.7 & -17.3 & 8.8 & 1.4 & -9.8 & -9.7 & $1.0 \%$ \\
\hline
\end{tabular}


B. NMR characterization of Scheme 1 intermediates (i.e. (2), (3), (4) and (5)), along with $\mathrm{FC}_{60}(\mathbf{1})$

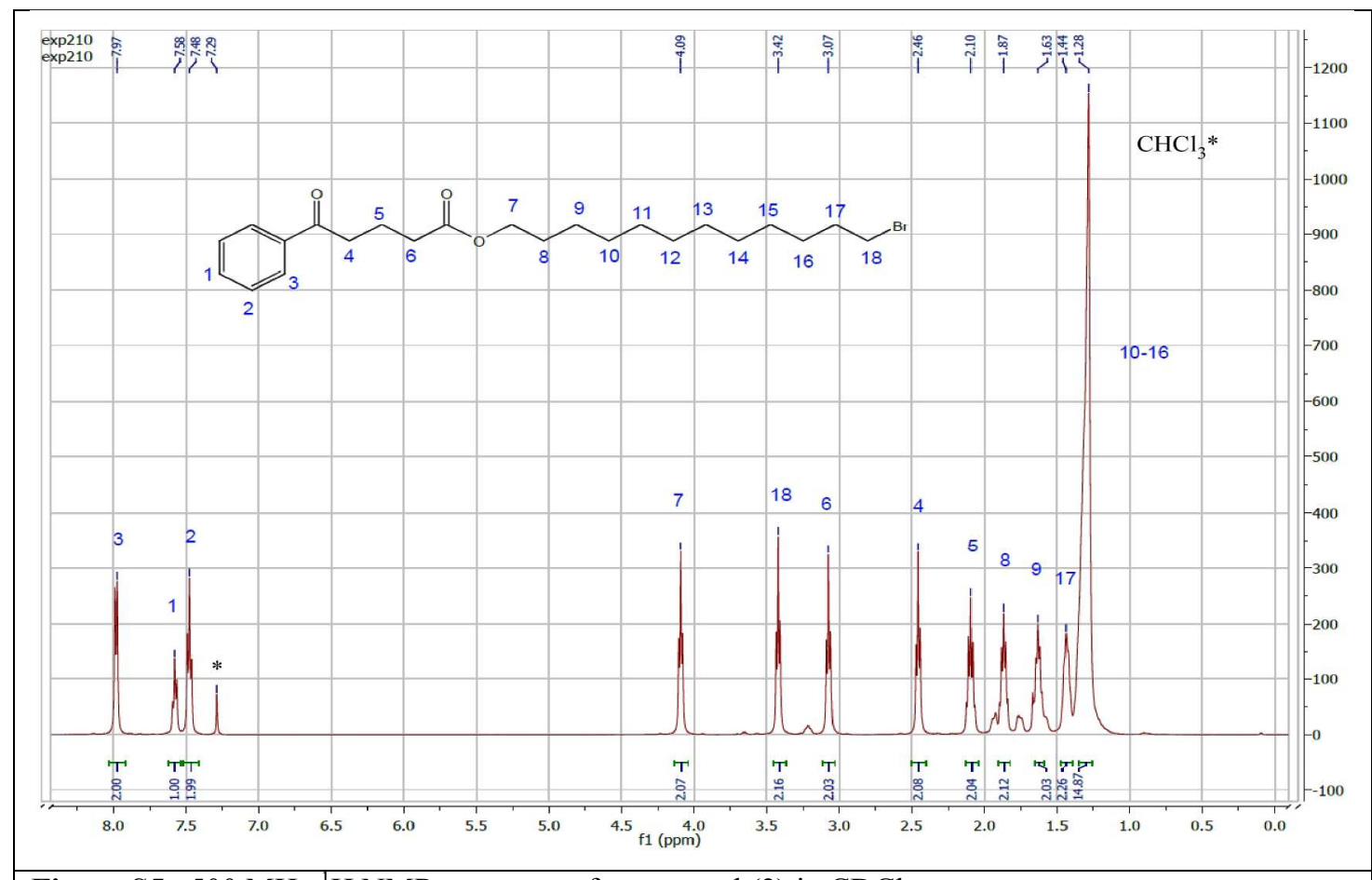

Figure S5. $500 \mathrm{MHz}{ }^{\mathrm{I}} \mathrm{H}$ NMR spectrum of compound (2) in $\mathrm{CDCl}_{3}$.

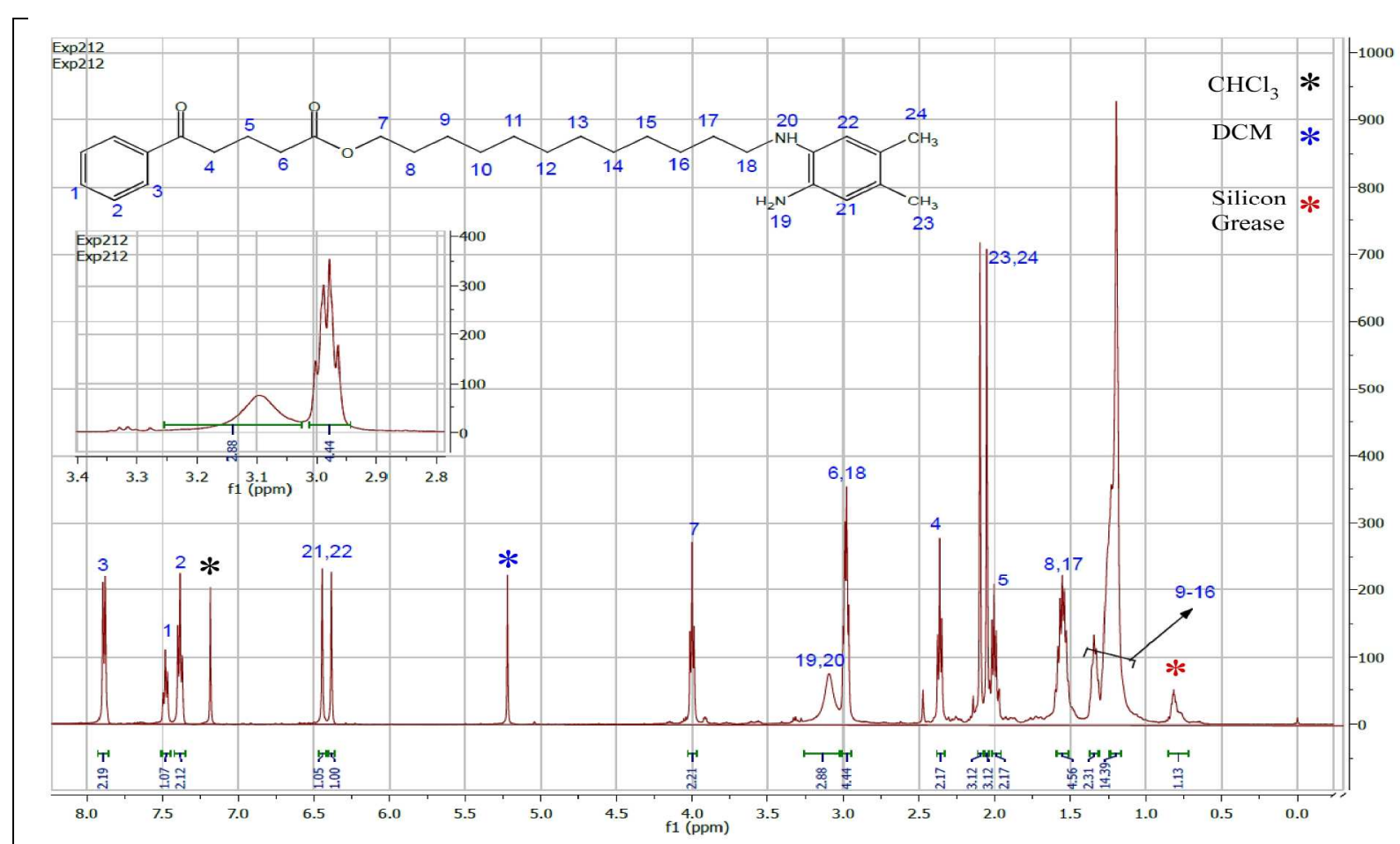

Figure S6. $500 \mathrm{MHz}{ }^{1} \mathrm{H}$ NMR spectrum of compound (3), in $\mathrm{CDCl}_{3}$. 

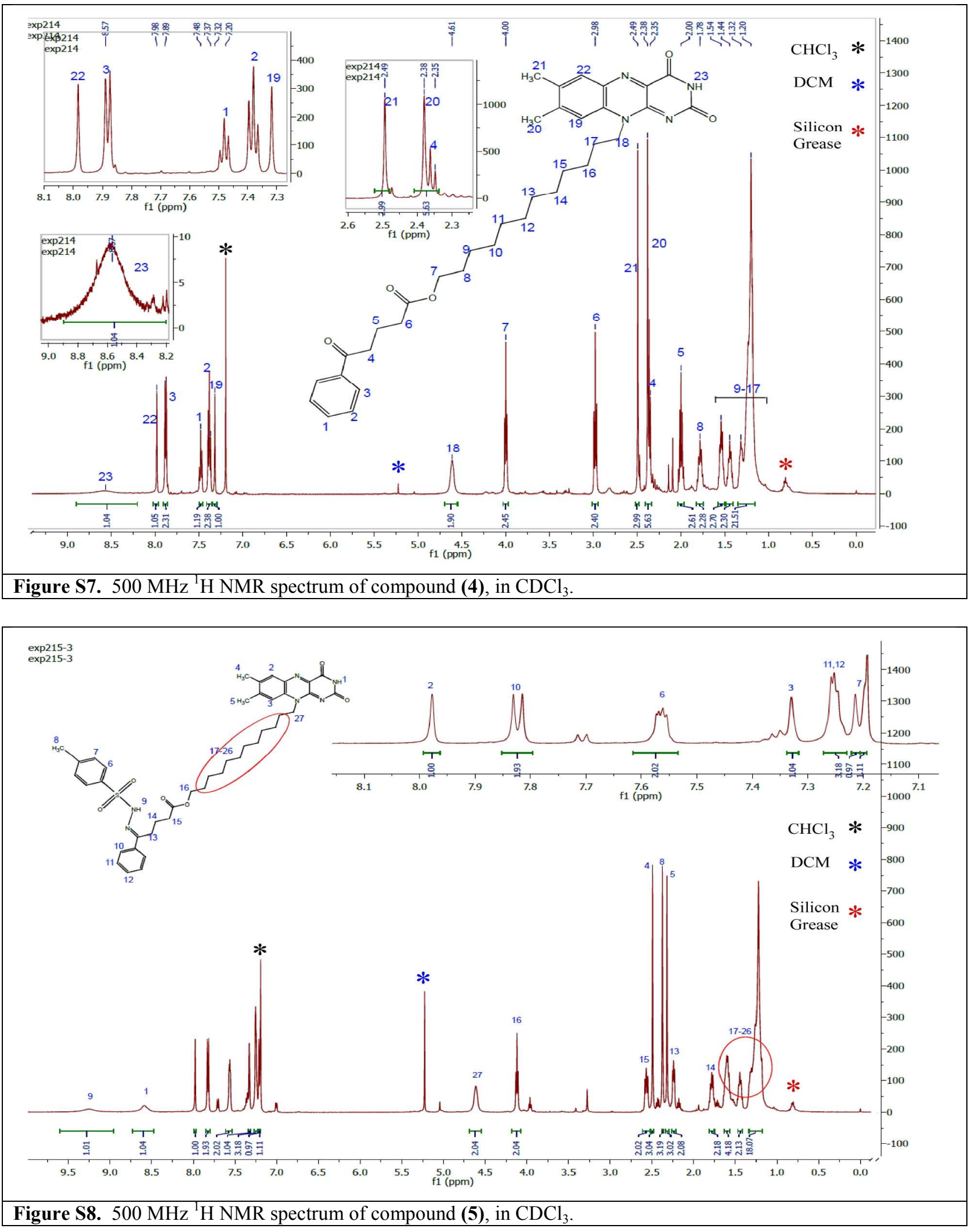


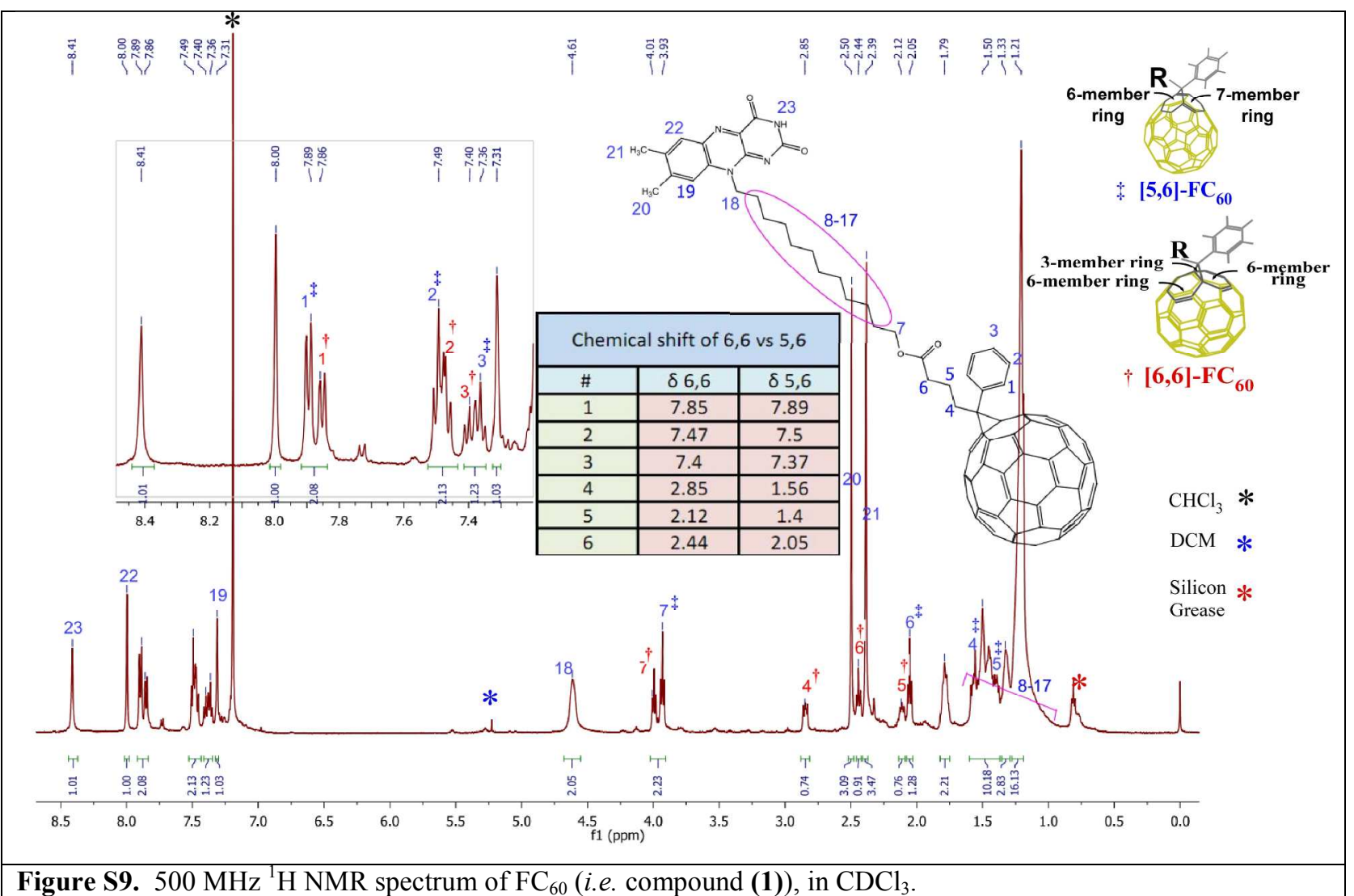

Figure S9. $500 \mathrm{MHz}{ }^{\mathrm{I}} \mathrm{H}$ NMR spectrum of $\mathrm{FC}_{60}$ (i.e. compound (1)), in $\mathrm{CDCl}_{3}$.

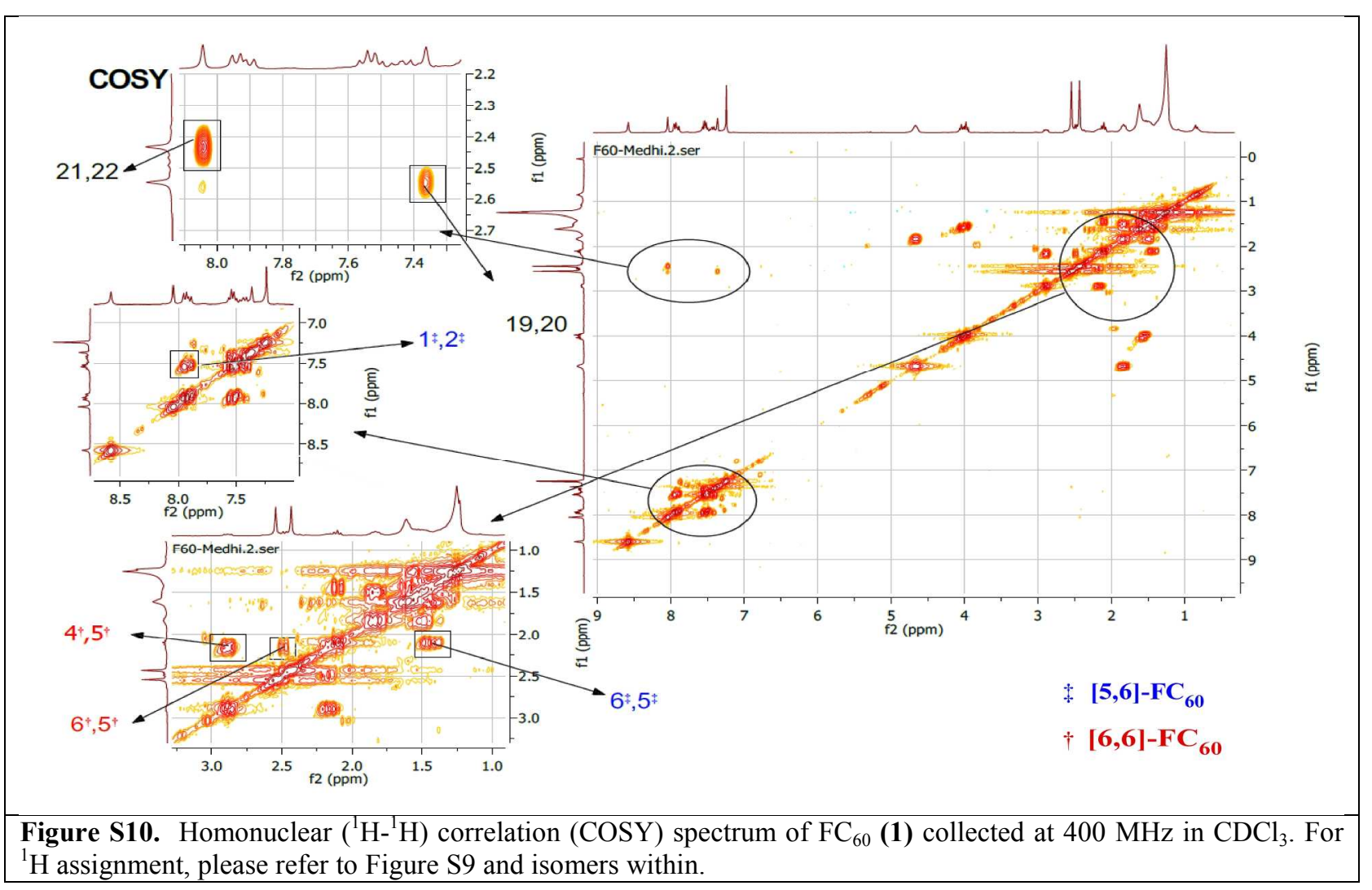


Table S18. Comparison of the aliphatic and aromatic domains of ${ }^{1} \mathrm{H}-\mathrm{NMR}$ for PCBM, intermediate (4) and $\mathrm{FC}_{60}$ (1).

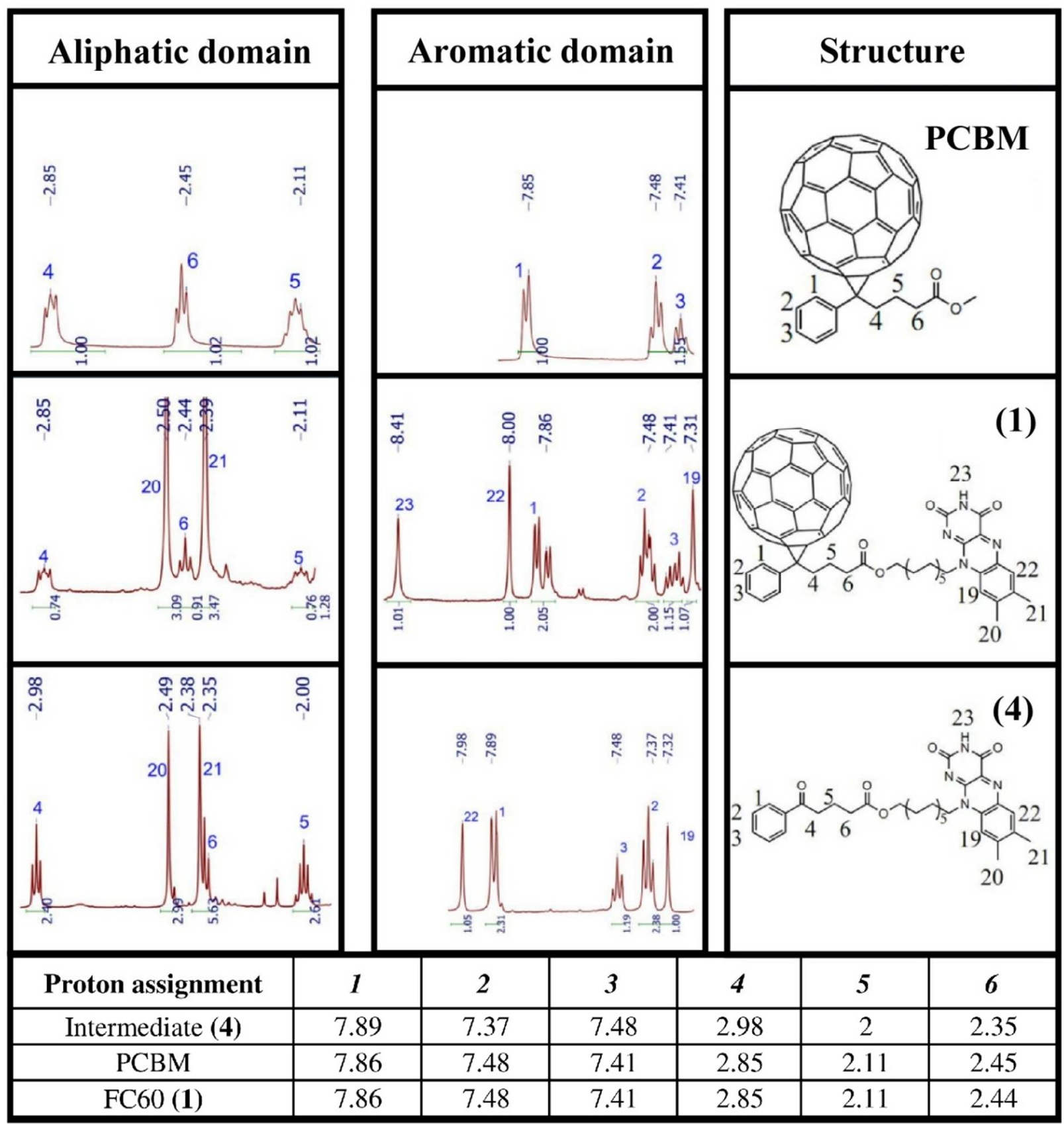




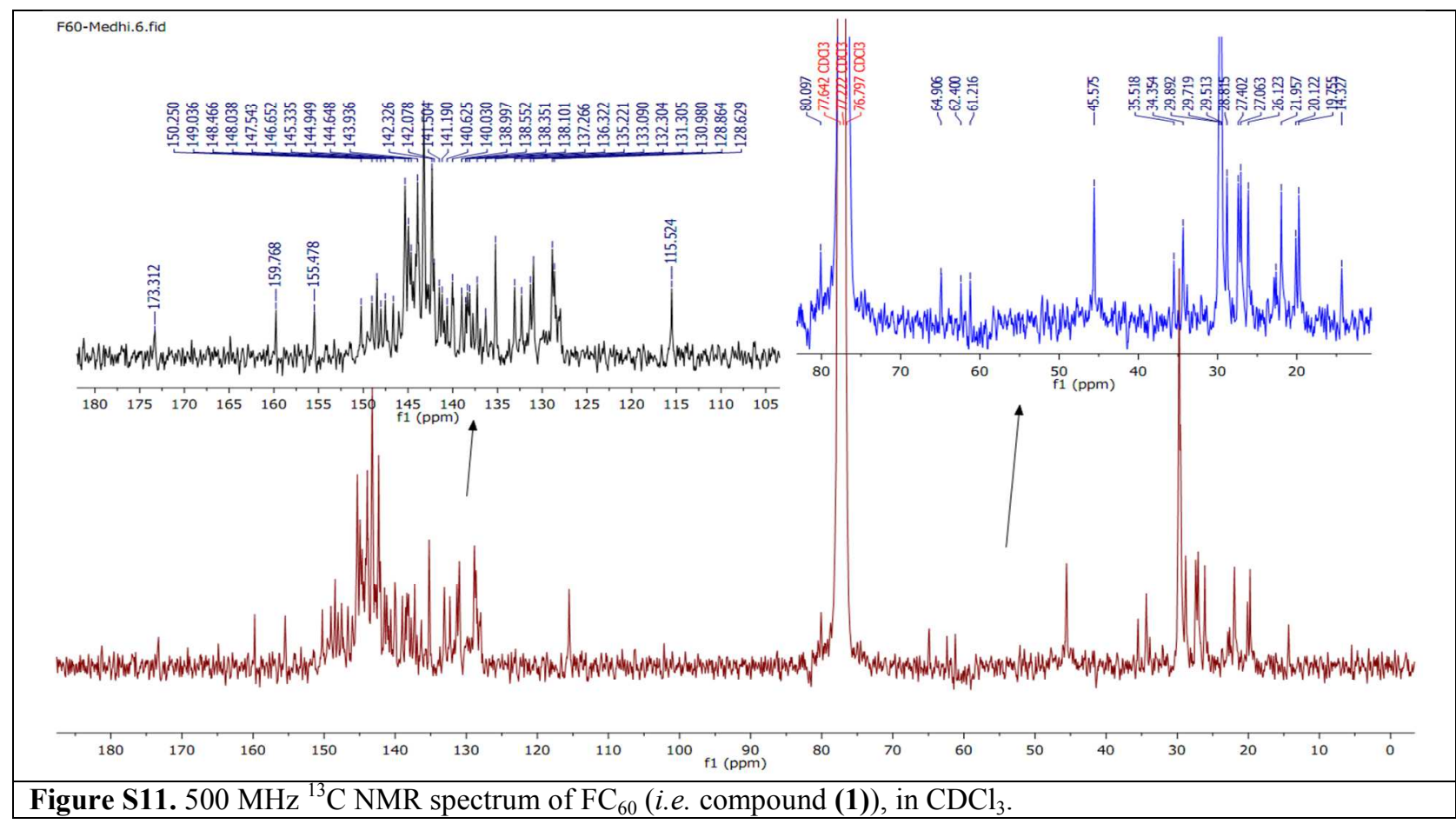




\section{Unsuccessful synthesis routes for the realization of $\mathrm{FC}_{60}(1)$}

Listed below are four unsuccessful synthesis routes for the realization of $\mathrm{FC}_{60}$. These unsuccessful synthesis routes hint on the possible involvement of a ground state complex between the flavin and fullerene precursors that eventually hinders their coupling.

\section{C.1. Unsuccessful route 1 (Trans-esterification)}

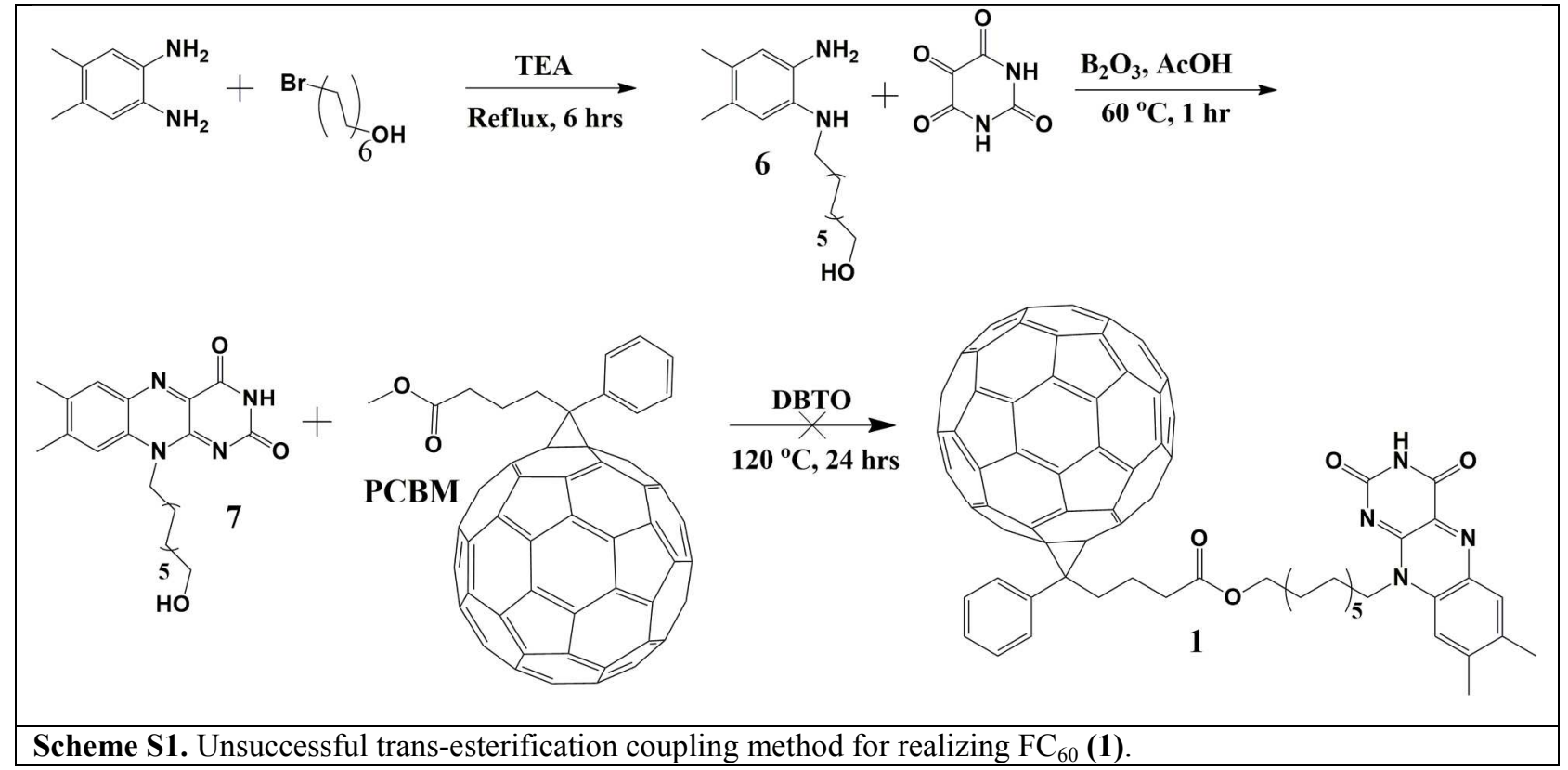

Synthesis of 4-((2-amino-4,5-dimethylphenyl) amino) dodecane-1-ol (6): A mixture of $1.00 \mathrm{~g}$ (30 mmol) of 4,5-dimethyl-1,2-phenylenediamine and $1.8 \mathrm{~g}(10 \mathrm{mmol})$ of 1-chloro-dodecane in $30 \mathrm{~mL}$ of triethylamine (TEA) was stirred at $90{ }^{\circ} \mathrm{C}$ for 6 hours under argon. After cooling and addition of dichloromethane $(100 \mathrm{~mL})$, the organic solution was washed with aqueous $\mathrm{Na}_{2} \mathrm{CO}_{3}$ solution $(10 \%, 40 \mathrm{~mL})$. The aqueous layer was extracted twice with dichloromethane $(2 \times 100$ $\mathrm{mL}$ ). The combined organic extracts were dried over $\mathrm{MgSO}_{4}$ and rotary evaporated to dryness. The thin-layer chromatography (TLC) retention factor $\left(R_{f}\right)$ of the target compound $\mathbf{6}$ was 0.55 with methylene chloride (DCM):methanol (MeOH) (95:5). Compound 6 was purified by flash chromatography on silica gel in DCM:MeOH (95:5) to produce $1.8 \mathrm{~g}$ of reddish crystals $(60 \%$ yield); ${ }^{1} \mathrm{H}$ NMR $\left(400 \mathrm{MHz}, \mathrm{CDCl}_{3}\right.$, see Figure S12) $\delta(\mathrm{ppm}) 6.56(1 \mathrm{H}, \mathrm{s}), 6.50(1 \mathrm{H}, \mathrm{s}), 3.20$ $(3 \mathrm{H}$, broad s), $3.1(2 \mathrm{H}, \mathrm{t}, \mathrm{J}=8 \mathrm{~Hz}), 2.26(3 \mathrm{H}, \mathrm{s}), 2.21(3 \mathrm{H}, \mathrm{s}), 1.68(2 \mathrm{H}, \mathrm{q}, \mathrm{J}=7 \mathrm{~Hz}), 1.46(2 \mathrm{H}$, $\mathrm{m}), 1.34(16 \mathrm{H}, \mathrm{s})$. 


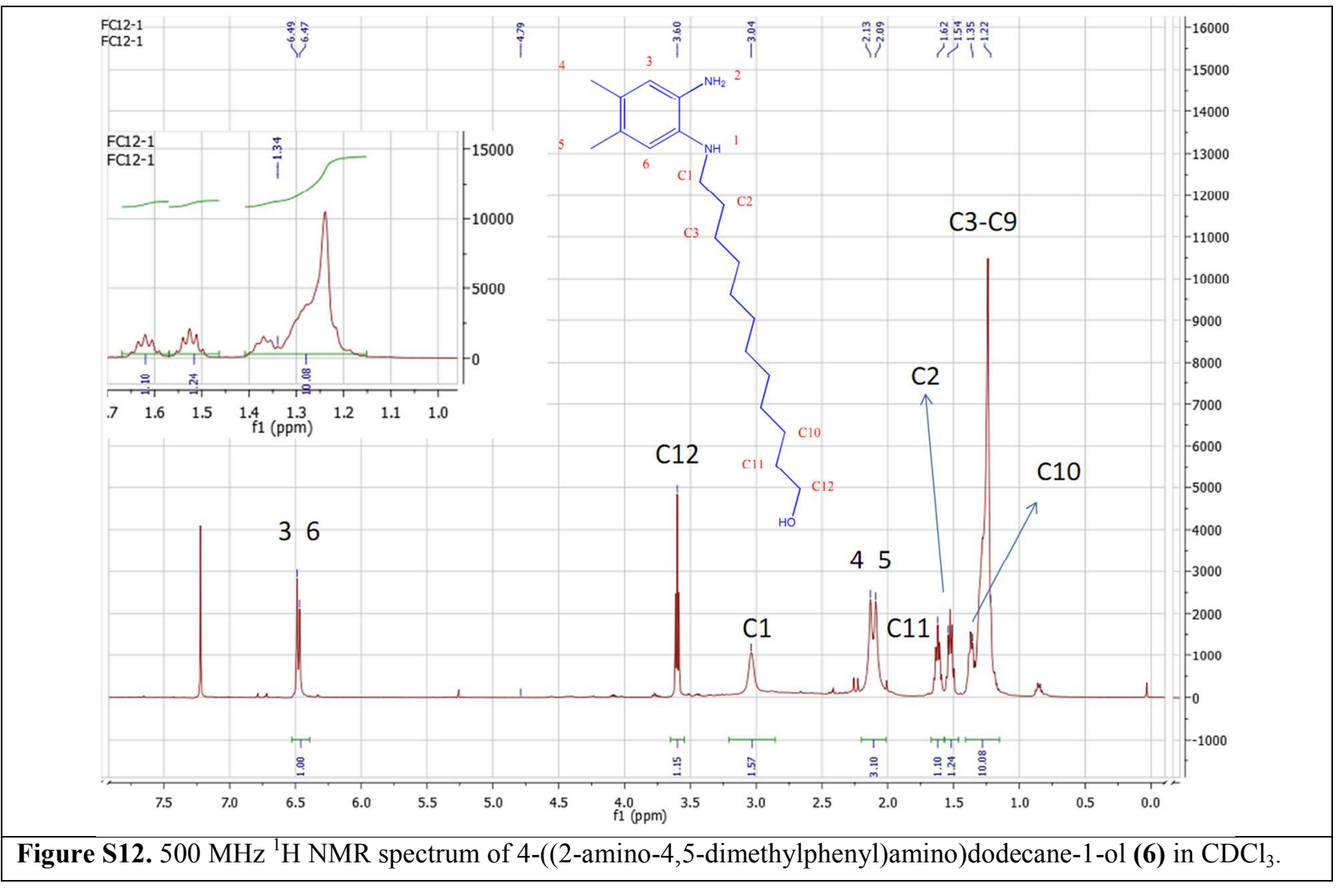

Synthesis of N-(12'-hydroxydodecanyl) isoalloxazine (FC12-OH (7)): A mixture of $0.578 \mathrm{~g}$ (1.9 $\mathrm{mmol})$ of compound (6), $0.284 \mathrm{~g}(2 \mathrm{mmol})$ of alloxane monohydrate, $0.42 \mathrm{~g}(6 \mathrm{mmol})$ of boric oxide and $60 \mathrm{~mL}$ of glacial acetic acid was stirred at $60^{\circ} \mathrm{C}$ for $1 \mathrm{hr}$. The resulting solution was quenched with $100 \mathrm{~mL}$ of water and the yellow precipitates were filtered and vacuum-dried. The $R_{f}$ value of FC12-OH by TLC was 0.85 with MC:MeOH (95:5). FC12-OH was purified by flash chromatography on silica gel in $\mathrm{MC}: \mathrm{MeOH}(95: 5)$ to produce $0.46 \mathrm{~g}$ of yellow crystals (58\% yield); mp 223-224 ${ }^{\circ} \mathrm{C} ;{ }^{1} \mathrm{H}$ NMR (400 $\mathrm{MHz} \mathrm{CDCl}_{3}$, see Figure S13) $\delta(\mathrm{ppm}) 8.85(1 \mathrm{H}, \mathrm{s}), 8.08$ $(1 \mathrm{H}, \mathrm{s}), 7.41(1 \mathrm{H}, \mathrm{s}), 4.71(2 \mathrm{H}$, broad t), $2.59(3 \mathrm{H}, \mathrm{s}), 2.48(3 \mathrm{H}, \mathrm{s}), 1.88(2 \mathrm{H}, \mathrm{q}, \mathrm{J}=8 \mathrm{~Hz}), 1.57$ $(2 \mathrm{H}, \mathrm{q}, \mathrm{J}=8 \mathrm{~Hz}), 1.51(2 \mathrm{H}, \mathrm{m}), 1.29(14 \mathrm{H}, \mathrm{s})$. 


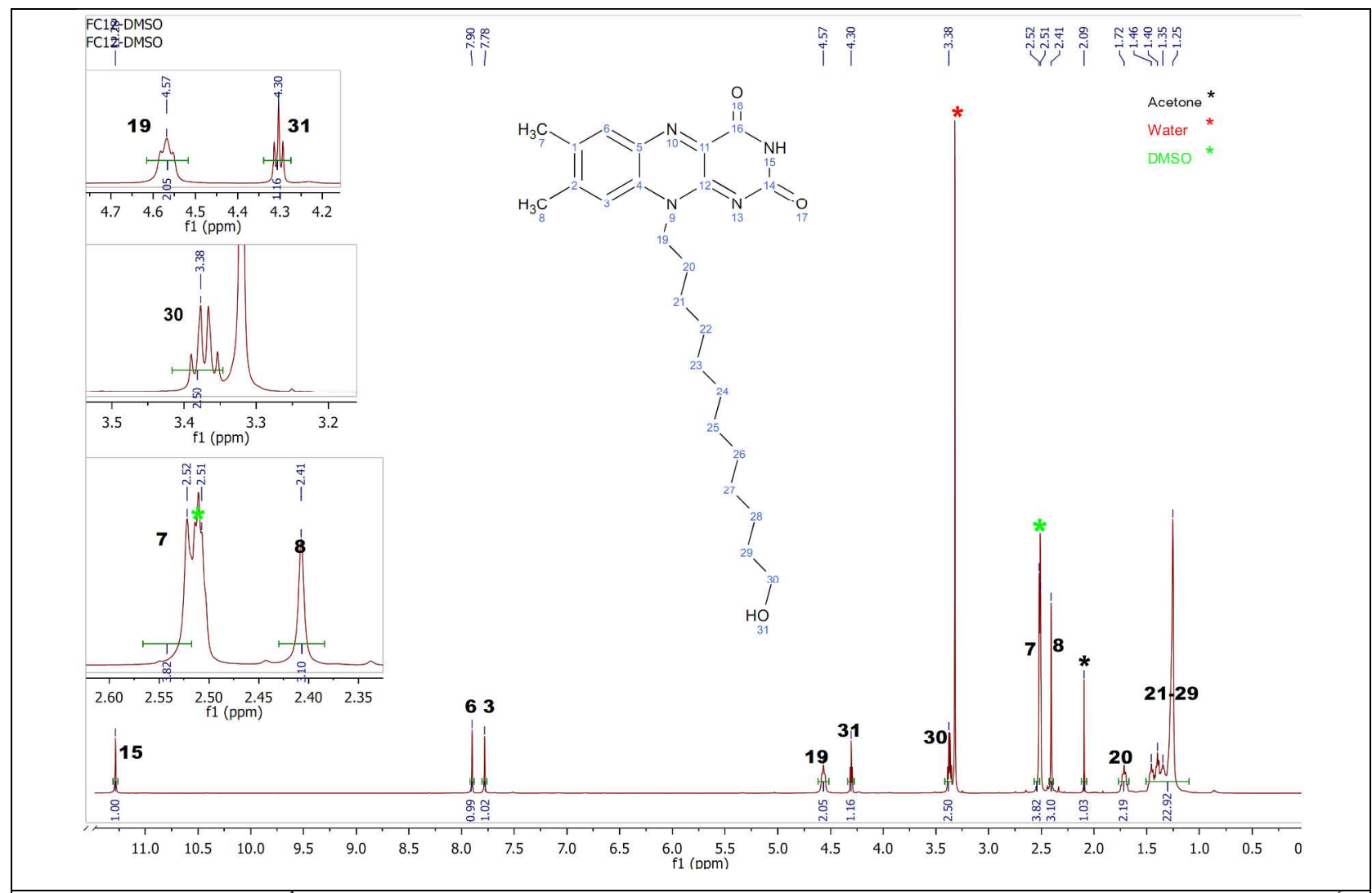

Figure S13. $500 \mathrm{MHz}{ }^{1} \mathrm{H}$ NMR spectrum of N-(12'-Hydroxydodecanyl) isoalloxazine (FC12-OH (7)), in DMSO-d ${ }^{6}$.

Unsuccessful synthesis of $\mathrm{C}_{60}-$ functionalized isoalloxazine (1): A $50 \mathrm{ml}$ flame dried threenecked flask was charged with [6,6]-phenyl- $\mathrm{C}_{61}$-butyric acid methyl ester (PCBM) (250 mg, $0.227 \mathrm{mmol})$ and dichlorobenzene $(10 \mathrm{ml})$. The resulting solution was degassed by three $\mathrm{N}_{2} /$ vacuum purges. Next, FC12-OH (7) (24 mg, $0.387 \mathrm{mmol}, 1.7$ eq.), and dibutyltinoxide (DBTO) (11.3 mg, $0.046 \mathrm{mmol}, 0.2$ eq.) were added. The mixture was stirred at $120{ }^{\circ} \mathrm{C}$ for 5 days. The desired trans-esterification reaction did not take place between the starting materials. After aging the reaction mixture, insoluble dark particles precipitated on the flask wall. The effort to dissolve and purify the unknown dark particles was not successful. 


\section{C.2. Unsuccessful route 2 (Steglich Esterification)}

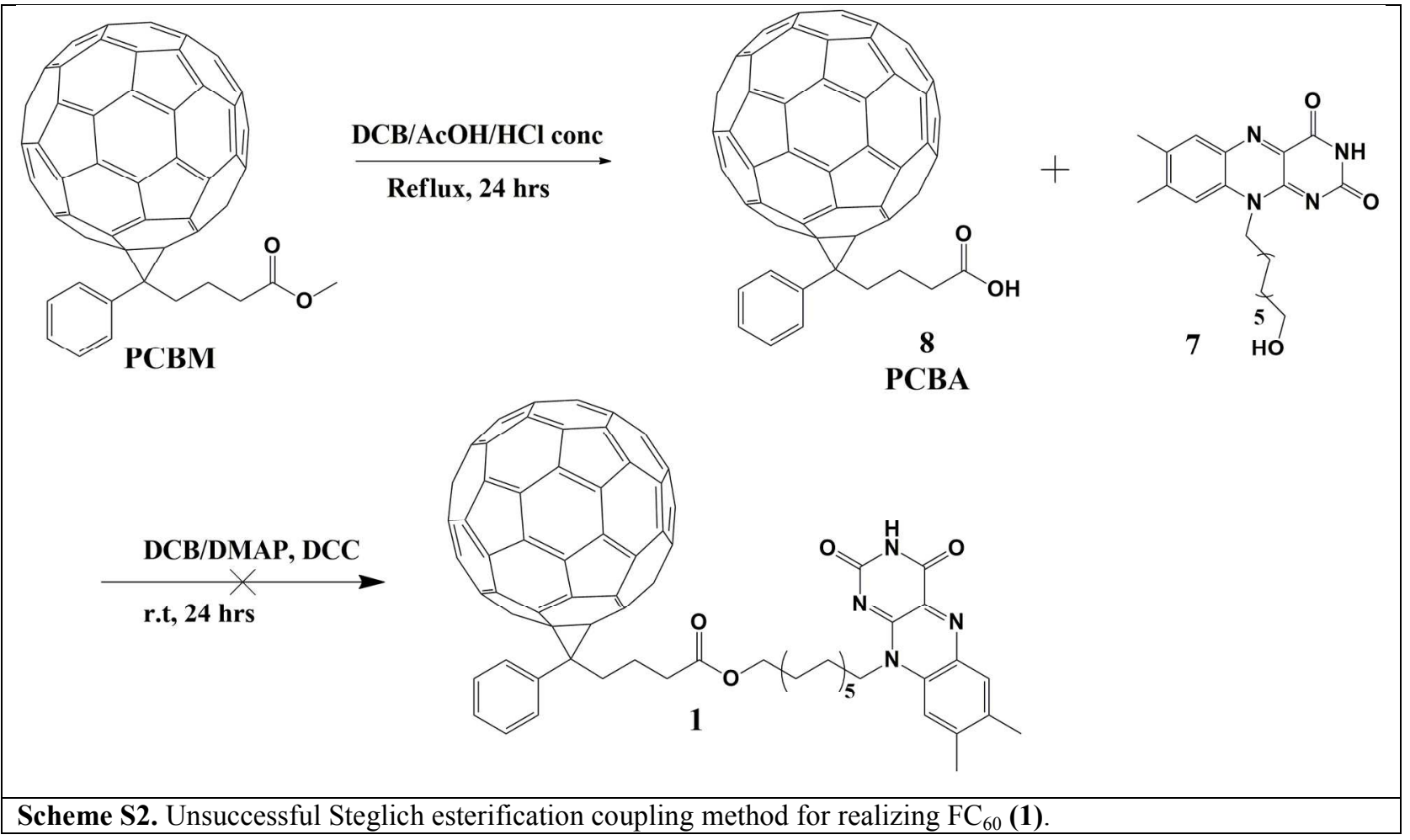

Preparation of [6,6]-phenyl-C61-butyric acid (PCBA) (8): To $100 \mathrm{~mL}$ of PCBM solution in toluene $(6 \mathrm{mg} / \mathrm{mL})$ was added glacial acetic acid $(50 \mathrm{~mL})$ and concentrated $\mathrm{HC} 1(20 \mathrm{~mL})$. The mixture was stirred and refluxed for 22 hours. The course of the reaction was followed by TLC. All volatile components were removed in vacuum. The residual solid was first washed with $\mathrm{MeOH}$, and then with a mixture of ether and toluene and dried in vacuum at $70{ }^{\circ} \mathrm{C}$ over a period of 16 hours. Yield: $97-99 \%$. The acid is sparingly soluble in $\mathrm{CS}_{2}$ and pyridine and more readily in a mixture of acetic acid with $\mathrm{CS}_{2} .{ }^{1} \mathrm{H}$ NMR $\left(\mathrm{CS}_{2} ; 500 \mathrm{MHz}\right.$, see Figure S3): $\delta(\mathrm{ppm}) 7.83$ (d, $\mathrm{J}=8 \mathrm{~Hz}, 2 \mathrm{H} ; \mathrm{O}-\mathrm{H}$ arom), 7.47 (m, 2H; m-H arom), 7.40 (m, 2H; p-H arom), 2.87 (m, 2H; $\mathrm{PhCCH}_{2}$ ), 2.47 (t, J=7 Hz, 2H; $\left.\mathrm{CH}_{2} \mathrm{CO}_{2}\right), 2.13\left(\mathrm{~m}, 2 \mathrm{H} ; \mathrm{CH}_{2} \mathrm{CH}_{2} \mathrm{CO}_{2}\right) .{ }^{13} \mathrm{CNMR}_{(\mathrm{CS}} ; 125 \mathrm{MHz}$; $145.1,145.0,144.8,144.7,144.5,144.2,143.8,143.14,143.10,143.03,142.97,142.2,142.1$, 142.06, 141.1, 140.9, 138.1, 137.7, 136.5, 132.0, 128.6 (PhC2,3), 128.4 (PhC2,3), 79.97 (bridgehead C), $52.06(\mathrm{PhC}), 34.23,34.11\left(\mathrm{CCO}_{2} \mathrm{H}\right.$ and $\left.\mathrm{PhCC}\right), 22.82\left(\mathrm{CCCO}_{2} \mathrm{H}\right)$. Anal. Calcd for $\mathrm{C}_{71} \mathrm{H}_{12} \mathrm{O}_{2}$ : C, 95.08; H, 1.35; Found: C, 92.40; H, 1.55. 


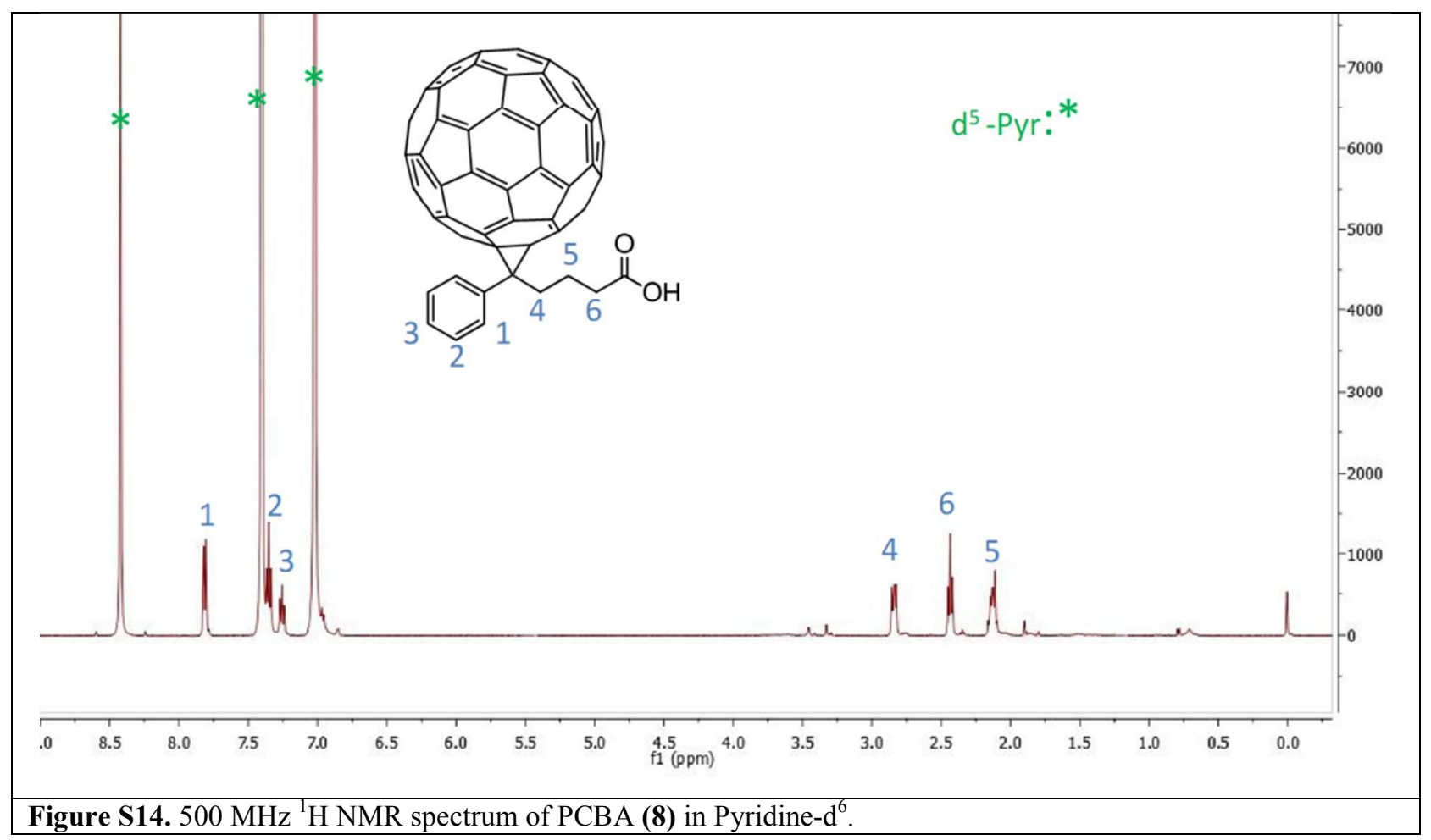

Unsuccessful synthesis of FC $\underline{60}_{0}$ (1): In a $100 \mathrm{~mL}$ round-bottom flask, $0.04 \mathrm{~g}$ of FC12-OH and PCBA (8), (0.045 g, $0.05 \mathrm{mmol})$, were added and the flask was degassed under vacuum and backfilled with argon gas. $6 \mathrm{~mL}$ of dichlorobenzene $/ \mathrm{CHCl}_{3}(50 / 50 \mathrm{v} / \mathrm{v})$ and $0.5 \mathrm{mg}$ of DMAP $(0.004 \mathrm{mmol})$ were added to the reaction mixture, and the solution was sonicated for $30 \mathrm{~min}$ to dissolve PCBA completely. Then, $10 \mathrm{mg}$ of 1,3-dicyclohexylcarbodiimide (DCC, $0.05 \mathrm{mmol}$ ) dissolved in $2 \mathrm{~mL}$ of dichlorobenzene $/ \mathrm{CHCl}_{3}(50 / 50 \mathrm{v} / \mathrm{v})$ and was added dropwise using a syringe, followed by stirring the solution at room temperature for 3 days. The course of the reaction was followed by TLC but no desired spot related to the product was observed after a few days aging of the reaction mixture. 


\section{C.3. Unsuccessful route 3 (Prato Reaction)}

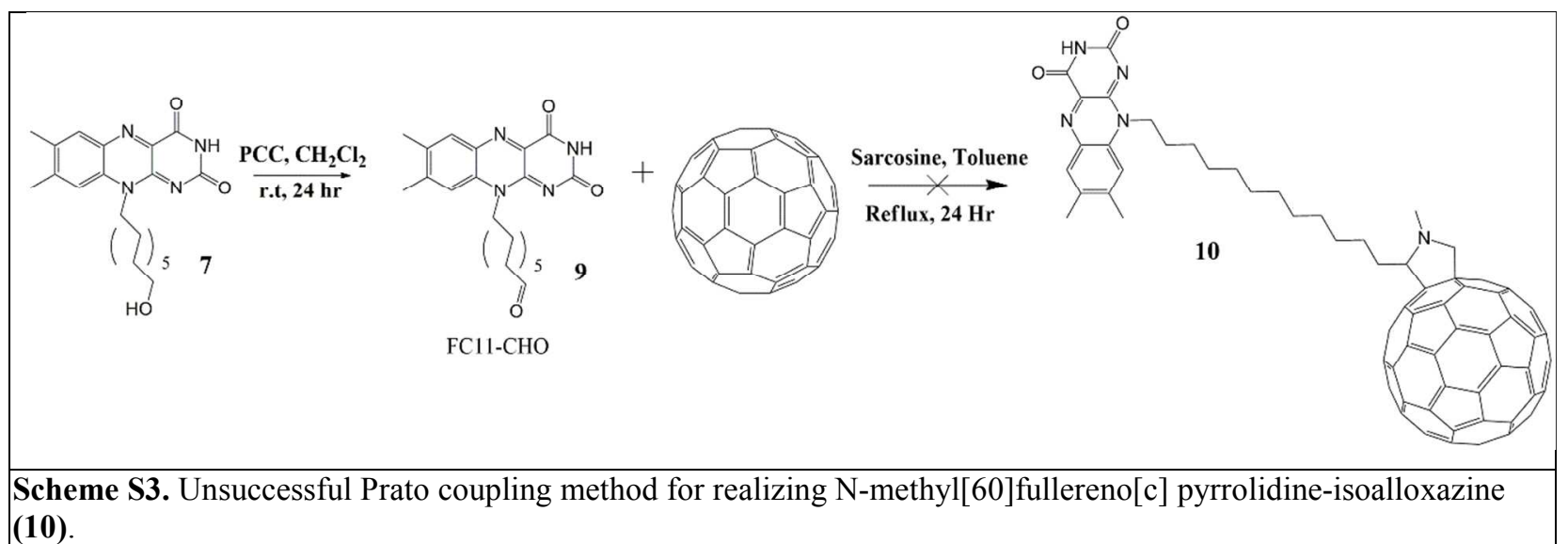

Synthesis of 10-(4-hydroxydodecenyl)-7,8-dimethylbenzo[g]pteridine-2,4(3H,10H)-dione

(FC12-CHO, 9): To a stirred solution of FC12-OH (7) $(0.5 \mathrm{~g}, 1.46 \mathrm{mmol})$ in dry $\mathrm{CH}_{2} \mathrm{Cl}_{2}(100$ $\mathrm{mL})$ pyridinium chlorochromate (PCC) $(0.63 \mathrm{~g}, 2.92 \mathrm{mmol})$ was added. The solution was stirred at room temperature for 4 hours. The resulting solution was poured into water $(100 \mathrm{~mL})$ and the lower organic layer was separated, dried over $\mathrm{MgSO}_{4}$, filtered and concentrated under reduced pressure. The crude mixture was purified using silica gel column chromatography (eluent: $\mathrm{CH}_{2} \mathrm{Cl}_{2}$ :Methanol, 19:1 (v/v)), to yield the aldehyde (FC12-CHO) (9) with 90\% yield. ${ }^{1} \mathrm{H}$ NMR $\left(\mathrm{CDCl}_{3}\right.$, see Figure S15): $\delta(\mathrm{ppm}) 9.73(1 \mathrm{H}, \mathrm{s}), 8.36(1 \mathrm{H}, \mathrm{s}), 8.00(1 \mathrm{H}, \mathrm{s}), 7.33(1 \mathrm{H}, \mathrm{s}), 4.65(2 \mathrm{H}$, t), $2.53(3 \mathrm{H}, \mathrm{s}), 2.47(2 \mathrm{H}, \mathrm{t}), 2.38(3 \mathrm{H}, \mathrm{s}), 1.78(2 \mathrm{H}, \mathrm{m}), 1.58(2 \mathrm{H}, \mathrm{m}), 1.5(2 \mathrm{H}, \mathrm{m}), 1.37(2 \mathrm{H}, \mathrm{m})$, $1.2(14 \mathrm{H}, \mathrm{m})$.

Unsuccessful synthesis of N-methyl[60]fullereno[c]pyrrolidine-isoalloxazine dyad (10): To a solution of $\mathrm{C}_{60}(0.21 \mathrm{~g}, 0.29 \mathrm{mmol})$ in toluene $(260 \mathrm{~mL})$, sarcosine $(0.05 \mathrm{~g}, 0.59 \mathrm{mmol})$ and (9) ( $1.6 \mathrm{~g}, 1.48 \mathrm{mmol})$ were added. The solution was then heated under reflux for 4 hours and then allowed to cool to room temperature. The resulting solution was concentrated under reduced pressure. The course of the reaction was followed by TLC. No desired product was obtained. 


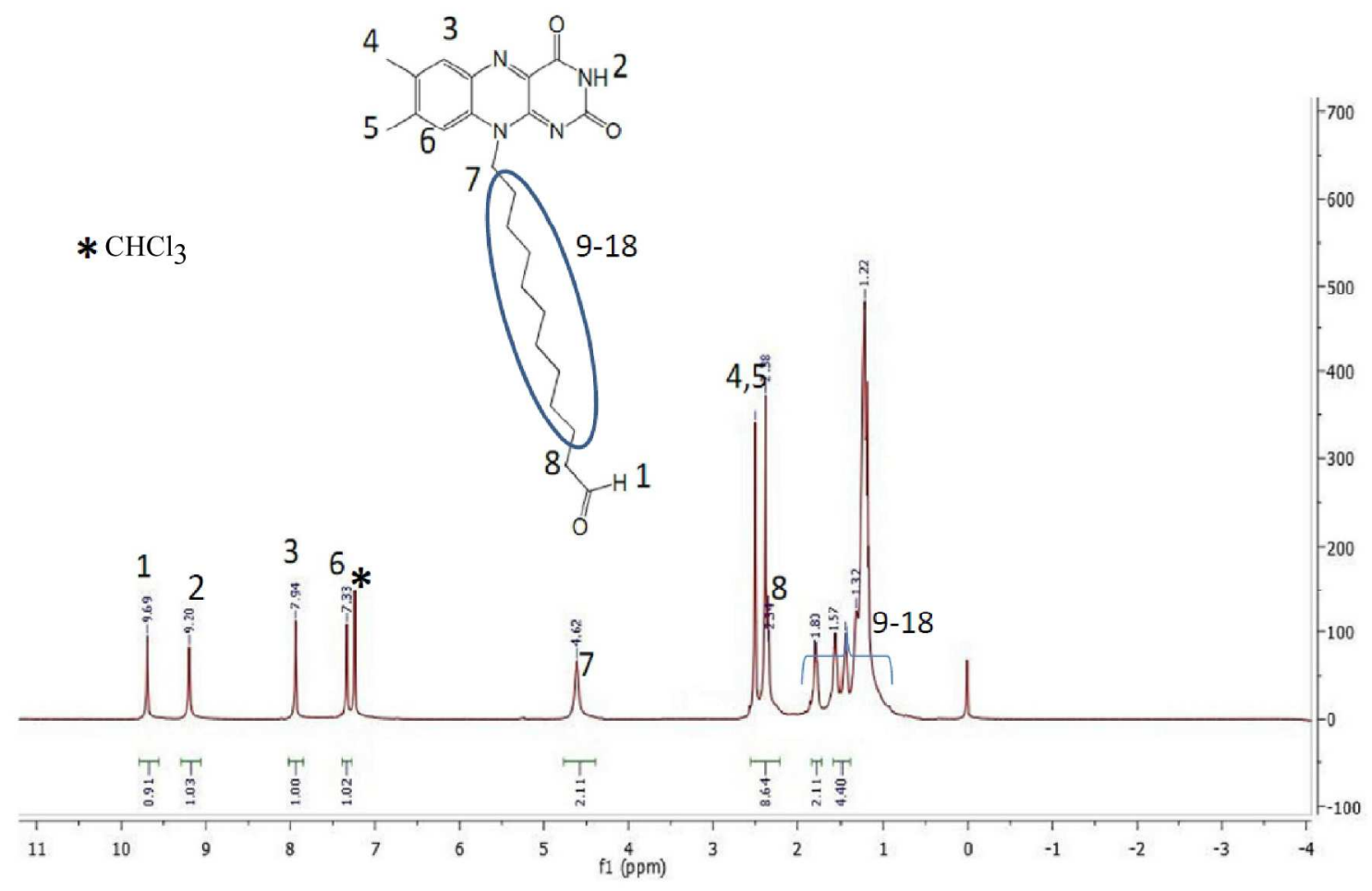

Figure S15. $500 \mathrm{MHz}^{1} \mathrm{H}$ NMR spectrum of $\mathrm{FC} 12-\mathrm{CHO}(9)$ in $\mathrm{CDCl}_{3}$. 


\section{C.4. Unsuccessful route 4 (Isoalloxazine building route)}

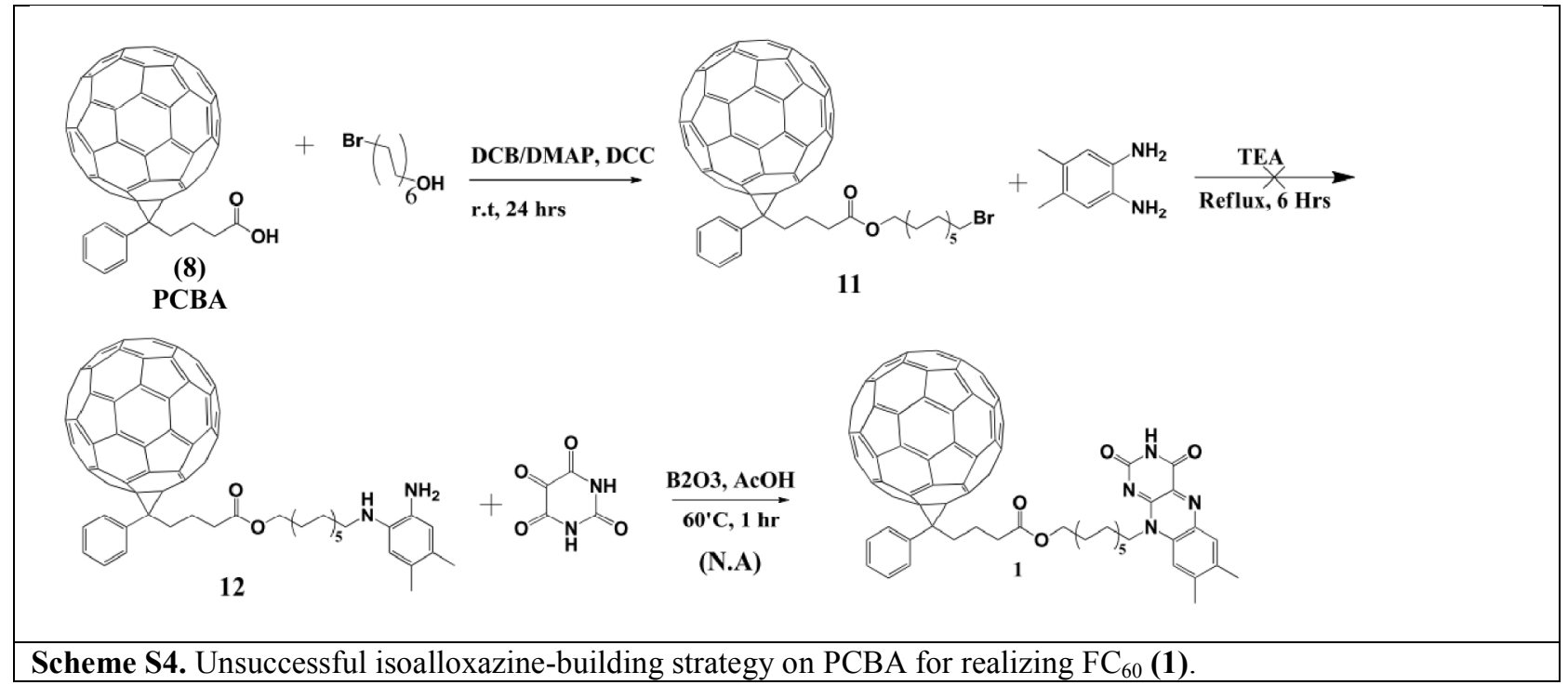

Preparation of 12-bromododecenyl [6,6]-phenyl-C 61 -butyrate (11): In a $100 \mathrm{~mL}$ round-bottom flask, $0.09 \mathrm{~g}$ of 12-bromo-1-dodecanol $(0.15 \mathrm{mmol})$ and PCBA (8) $(0.1 \mathrm{~g}, 0.05 \mathrm{mmol})$ were added and the flask was degassed under vacuum and backfilled with argon gas. $10 \mathrm{~mL}$ of dichlorobenzene/ $\mathrm{CHCl}_{3}(50 / 50 \mathrm{v} / \mathrm{v})$ and $0.5 \mathrm{mg}$ of DMAP $(0.004 \mathrm{mmol})$ were added to the reaction mixture, and the solution was sonicated for $30 \mathrm{~min}$ to dissolve PCBA completely. Then, $10 \mathrm{mg}$ of 1,3-dicyclohexylcarbodiimide (DCC, $0.05 \mathrm{mmol}$ ) dissolved in $2 \mathrm{~mL}$ of dichlorobenzene/ $\mathrm{CHCl}_{3}(50 / 50 \mathrm{v} / \mathrm{v})$ was added dropwise using a syringe, and the solution was stirred at room temperature for 1 day. The course of the reaction was followed by TLC. The solvent was removed by applying vacuum and then the product was purified using silica gel column chromatography (eluent: $\mathrm{CH}_{2} \mathrm{Cl}_{2}$ :Methanol, 19:1 (v/v)). Yield: $193 \mathrm{mg}(85 \%)$. ${ }^{1} \mathrm{H}$ NMR $\left(500 \mathrm{MHz}, \mathrm{CDCl}_{3}\right.$, see Figure S16) $\delta(\mathrm{ppm}): 7.87(2 \mathrm{H}, \mathrm{d}), 7.50(2 \mathrm{H}, \mathrm{t}), 7.37(1 \mathrm{H}, \mathrm{t}), 6.51(1 \mathrm{H}, \mathrm{s})$, $6.51(1 \mathrm{H}, \mathrm{s}), 4.61(2 \mathrm{H}$, broad s), $3.93(2 \mathrm{H}, \mathrm{t}), 2.84(2 \mathrm{H}, \mathrm{t}), 2.49(3 \mathrm{H}, \mathrm{s}), 2.44(2 \mathrm{H}, \mathrm{t}), 2.38(3 \mathrm{H}, \mathrm{s})$, $2.06(2 \mathrm{H}, \mathrm{m}), 1.78(2 \mathrm{H}, \mathrm{m}), 1.58(2 \mathrm{H}, \mathrm{m}), 1.5(2 \mathrm{H}, \mathrm{m}), 1.37(2 \mathrm{H}, \mathrm{m}), 1.2(14 \mathrm{H}, \mathrm{m})$. 


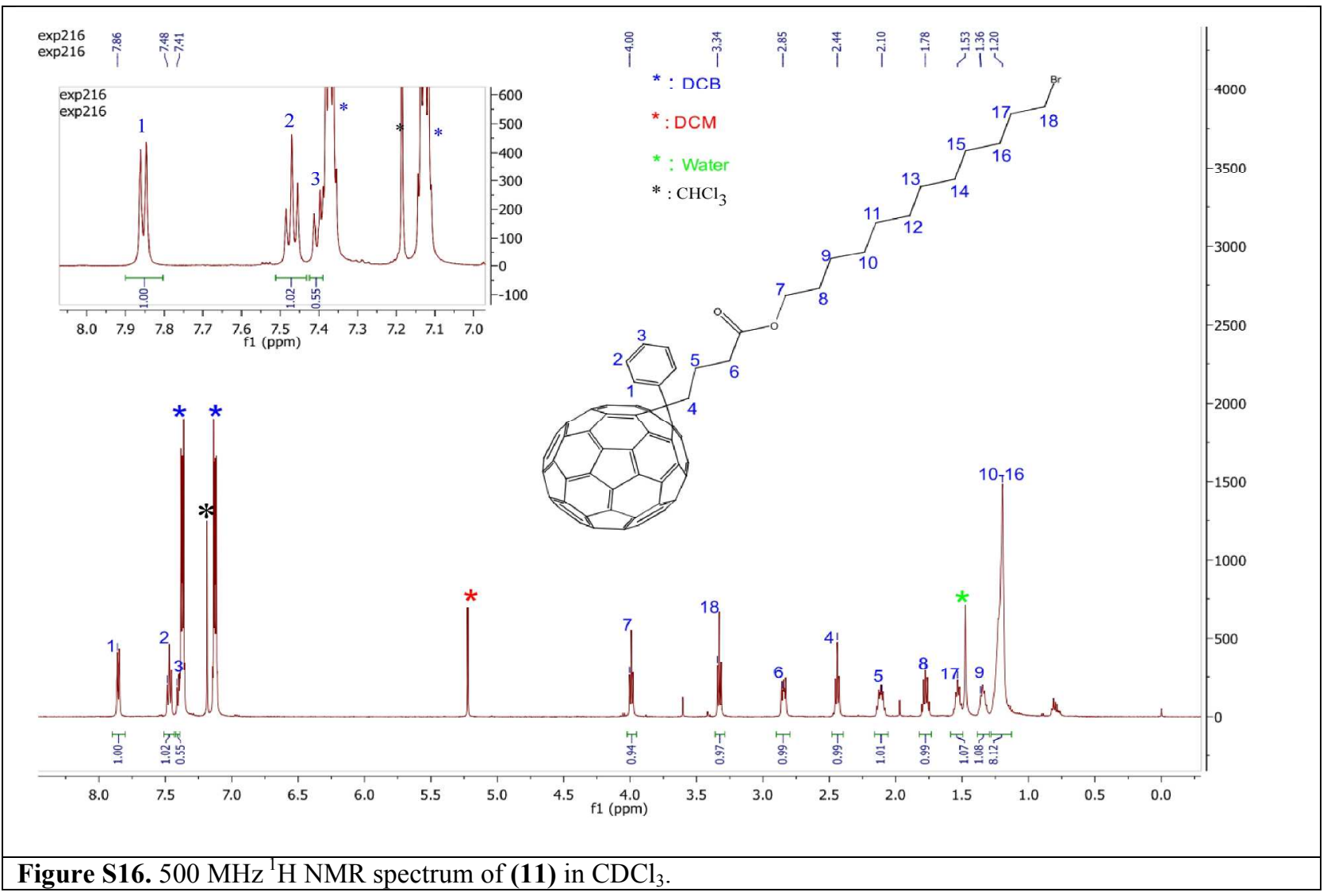

Preparation of 12-((2-amino-4,5-dimethylphenyl)amino)dodecyl-[6,6]-phenyl-C 61 -butyrate (12) : A mixture of $0.1 \mathrm{~g}(30 \mathrm{mmol})$ of 4,5-dimethyl-1,2-phenylenediamine and $0.1 \mathrm{~g}(10 \mathrm{mmol})$ of intermediate 5 in $30 \mathrm{~mL}$ of triethylamine was stirred at $90{ }^{\circ} \mathrm{C}$ for $6 \mathrm{hrs}$ under argon. The course of the reaction was followed by TLC. No desired product was obtained. 


\section{FTIR determination of isoalloxazine/ $\mathbf{C}_{\mathbf{6 0}}$ charge transfer interactions in $\mathbf{F C}_{\mathbf{6 0}}$}

According to Mulliken's theory, ${ }^{1}$ IR shifts should be observed in vibration bands that are affected by charge transfer. Such spectral shift $(\Delta v)$ can assist in determining the degree of charge transfer $(\delta)$ between donor and acceptor. If $\Delta v$ is small, then the following equation applies, ${ }^{2}$

$$
\delta=2 \Delta v / v_{o} \cdot\left[1-\left(v_{l} / v_{o}\right)^{2}\right]^{-1}
$$

where $v_{o}$ and $v_{l}$ correspond to the peak positions of the vibration of interest for the neutral and radical-anion (or cation), respectively. Because of a significant overlap with absorptions from PCBM and FC12, the determination of $\Delta v$ is very difficult for the $\mathrm{C}_{60}$ moiety. On the other hand, assessing $\Delta v$ for the flavin moiety is more straight forward due to its sharp $\mathrm{C}=\mathrm{N}$ stretch (i.e. inphase $\left(1580 \mathrm{~cm}^{-1}\right)$ and out-of-phase $\left(1507 \mathrm{~cm}^{-1}\right)$, respectively). ${ }^{3,4,5}$ The molecular simulation results of Figure 3 (in the main text) indicate that both $\mathrm{C}=\mathrm{N}$ bands are ideal candidates for accessing the magnitude of $\mathrm{CT}$ between $\mathrm{C}_{60}$ and isoalloxazine ring. Moreover, since the in-phase $\mathrm{C}=\mathrm{N}$ stretch is known for the radical-anion (i.e. $1543 \mathrm{~cm}^{-1}$ ) of lumiflavin, ${ }^{5,6}$ this value was chosen for $v_{1}$ and $1580 \mathrm{~cm}^{-1}$ for $v_{o}$. Correspondingly, the $\Delta v$ of $5 \mathrm{~cm}^{-1}$ for both $\mathrm{FC}_{60}$ and isomolar $\mathrm{PCBM} / \mathrm{FC} 12$ mixture amounts to a $\delta$ of 0.14 electrons.

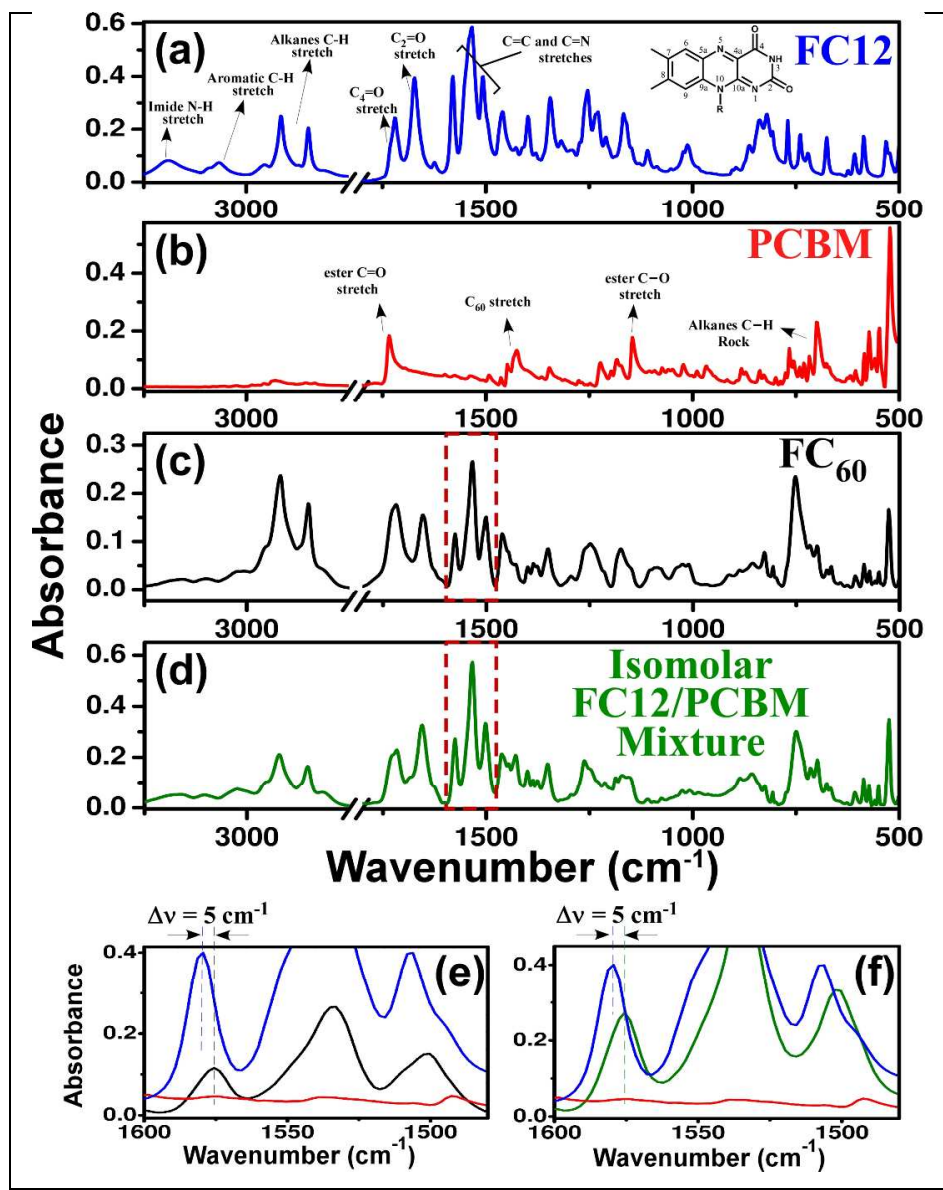

Figure S17. FTIR spectra of (a) FC12 (blue), (b) PCBM (red), (c) $\mathrm{FC}_{60}$ (black) and (d) isomolar mixture of PCBM and FC12 (green). The bottom two spectra illustrate the blown-up region of interest (within dashed red-box), where $\mathrm{FC}_{60}$ (e) and isomolar $\mathrm{PCBM} / \mathrm{FC} 12$ mixture (f) is overlaid together with PCBM and FC12 spectra. 


\section{E. $(n, m)$-characterization of FC12-dispersed SWNTs in the presence and absence of $\mathrm{FC}_{60}$ and $\mathrm{C}_{60}$}

SWNT dispersion protocol with pure FC12: A mixture of $1 \mathrm{mg}$ of HiPco SWNTs and $4 \mathrm{mg}$ of FC12 were cup-horn sonicated in $4 \mathrm{ml}$ of toluene for $1 \mathrm{hr}$ at $300 \mathrm{~W}$ intensity. The resulting greenish-dark suspension was centrifuged at $5 \mathrm{~kg}$ for $30 \mathrm{~min}$ and the supernatant (upper $90 \%$ ) was carefully collected, to remove any bundled carbon nanotubes, to afford a clear greenish-dark SWNT suspension.

Preparation of FC12-dispersed SWNTs in the presence of 1,2 and $3 \% \mathrm{FC}_{60}: 1,2$ and $3 \% \mathrm{FC}_{60}$, based on the molar concentration of FC12 used to disperse SWNTs, was added to the afforementioned FC12-dispersed SWNT suspension in toluene and bath sonicated for 30 min to ensure complete dispersion.

Preparation of FC12-dispersed SWNTs in the presence of $5 \% \mathrm{C}_{60}: 5 \% \mathrm{C}_{60}$, based on the molar concentration of FC12 used to disperse SWNTs, was added to the afforementioned FC12dispersed SWNT suspension in toluene and bath sonicated for $30 \mathrm{~min}$ to ensure complete dispersion.

$(n, m)$-SWNT characterization of the resulting dispersions before and after annealing at $55^{\circ} \mathrm{C}$ : Following background correction (by subtracting a power-law function $\left(\mathrm{a} \lambda^{-\mathrm{b}}\right)$, where $\lambda$ is the wavelength and $\mathrm{a}, \mathrm{b}$ are fitted parameters ${ }^{7}$ ) the NIR $E_{11}{ }^{S}$ absorption spectra of the aforementioned suspensions were deconvoluted using Lorentzian peak profiles to produce an optimum fit. Figure S18 illustrates the deconvoluted $E_{11} S$ absorption spectra of semiconducting $(n, m)$-SWNTs dispersed in FC12, and molar mixtures of $1 \% \mathrm{FC}_{60}+99 \% \mathrm{FC} 12$ and $5 \% \mathrm{C}_{60}+$ $95 \% \mathrm{FC} 12$, before and after annealing at $55^{\circ} \mathrm{C}$ for 12 hours. The PLE map of FC12-wrapped SWNTs is also included in Figure S18 for reference.

Tabulated peak-centers and full-width-half-maxima (FWHM) are shown in Tables S19. The percantage of peak center change (redshift) before and after annealing for different solutions of FC12-wrapped SWNTs, $+5 \% \mathrm{C}_{60}$ and $+1 \% \mathrm{FC}_{60}$ (derived from Table S19) are plotted in Figure S19.

Improving helix perfection (upon annealling at $55^{\circ} \mathrm{C}$ for 12 hours) provides a better shielding from direct $\mathrm{FC}_{60}$ and $\mathrm{C}_{60}$ contacts with the underlying SWNT species, which supresses the magnitude of red shift. $1 \%$ incorporation of $\mathrm{FC}_{60}$ triples the magnitude of red shift compared to $5 \% \mathrm{C}_{60}$. This provides a clear indication of the importance of flavin moiety in localizing the fullerene in the viscinity of SWNTs. 


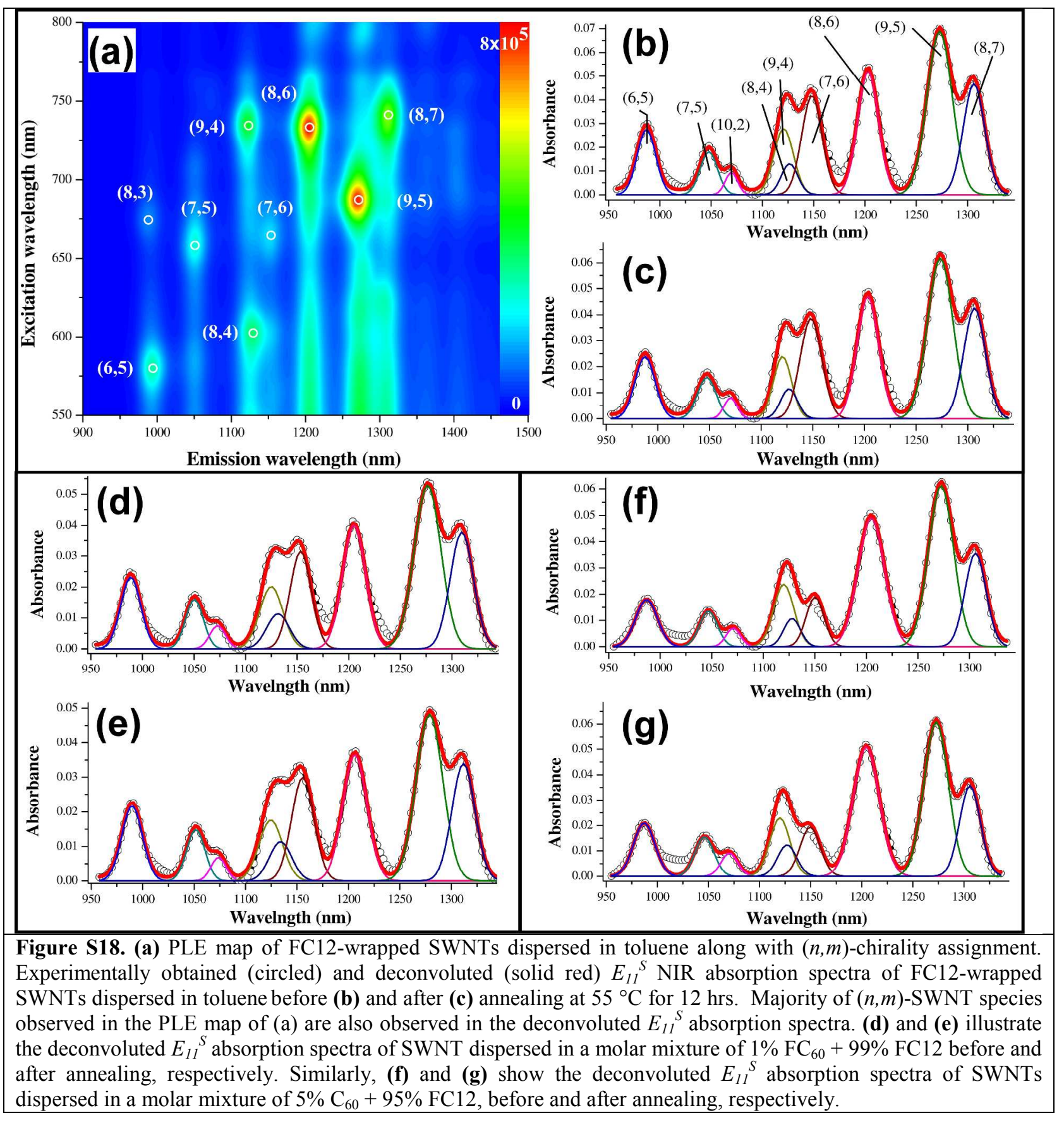


Table S19. $E_{11}{ }^{S}$ deconvoluted absorption maxima $\left(E_{M a x}\right)$ and full-width-at-half-maximum (FWHM) values of FC12wrapped SWNTs, and molar mixtures of $1 \% \mathrm{FC}_{60}+99 \% \mathrm{FC} 12$ and $5 \% \mathrm{C}_{60}+95 \% \mathrm{FC} 12$, before (a) and after (b) annealing at $55^{\circ} \mathrm{C}$ for 12 hours (data derived from Figure S18).

\begin{tabular}{|c|c|c|c|c|c|c|}
\hline \multicolumn{7}{|c|}{ (a) As dispersed } \\
\hline \multirow[b]{2}{*}{$\begin{array}{l}\text { Nanotube } \\
\text { assignments } \\
\quad(n, m)\end{array}$} & \multicolumn{3}{|c|}{$E_{M a x}(\mathrm{~nm})$} & \multicolumn{3}{|c|}{ FWHM (nm) } \\
\hline & $\begin{array}{l}\text { FC12- } \\
\text { wrapped } \\
\text { SWNTs }\end{array}$ & $\begin{array}{c}+1 \% \\
\mathrm{FC}_{60}\end{array}$ & $\begin{array}{c}+5 \% \\
\mathrm{C}_{60}\end{array}$ & $\begin{array}{c}\text { FC12- } \\
\text { wrapped } \\
\text { SWNTs }\end{array}$ & $\begin{array}{c}+1 \% \\
\mathrm{FC}_{60}\end{array}$ & $\begin{array}{c}+5 \% \\
\mathrm{C}_{60}\end{array}$ \\
\hline$(6,5)$ & 986.95 & 989.77 & 987.96 & 21.15 & 23.59 & 22.44 \\
\hline$(7,5)$ & 1047.09 & 1051.71 & 1048.87 & 19.75 & 22.76 & 21.84 \\
\hline$(10,2)$ & 1069.76 & 1075.00 & 1072.13 & 14.16 & 18.23 & 17.85 \\
\hline$(9,4)$ & 1120.70 & 1125.59 & 1123.57 & 22.54 & 27.13 & 24.62 \\
\hline$(8,4)$ & 1126.49 & 1134.06 & 1130.13 & 19.05 & 25.60 & 24.05 \\
\hline$(7,6)$ & 1148.25 & 1155.65 & 1150.52 & 23.24 & 26.66 & 24.82 \\
\hline$(8,6)$ & 1202.95 & 1206.29 & 1204.70 & 23.94 & 27.84 & 25.18 \\
\hline$(9,5)$ & 1272.79 & 1278.83 & 1274.84 & 28.83 & 31.66 & 29.09 \\
\hline$(8,7)$ & 1306.21 & 1311.82 & 1308.69 & 24.64 & 27.42 & 25.62 \\
\hline
\end{tabular}

\begin{tabular}{|c|c|c|c|c|c|c|}
\hline \multicolumn{7}{|c|}{ (b) After 12-hr annealing at 55 ${ }^{\circ} \mathrm{C}$} \\
\hline \multirow{3}{*}{$\begin{array}{c}\text { Nanotube } \\
\text { assignments } \\
(n, m)\end{array}$} & \multicolumn{3}{|c|}{$E_{\text {Max }}(\mathrm{nm})$} & \multicolumn{3}{c|}{ FWHM (nm) } \\
\cline { 2 - 7 } & $\begin{array}{c}\text { FC12- } \\
\text { wrapped } \\
\text { SWNTs }\end{array}$ & $\begin{array}{c}+1 \% \\
\mathrm{FC}_{60}\end{array}$ & $\begin{array}{c}+5 \% \\
\mathrm{C}_{60}\end{array}$ & $\begin{array}{c}\text { FC12- } \\
\text { wrapped } \\
\text { SWNTs }\end{array}$ & $\begin{array}{c}+1 \% \\
\mathrm{FC}_{60}\end{array}$ & $\begin{array}{c}+5 \% \\
\mathrm{C}_{60}\end{array}$ \\
\hline$(6,5)$ & 986.65 & 988.71 & 987.09 & 21.51 & 22.90 & 22.12 \\
\hline$(7,5)$ & 1047.06 & 1050.48 & 1048.45 & 20.30 & 22.06 & 21.04 \\
\hline$(10,2)$ & 1070.44 & 1072.87 & 1071.47 & 14.99 & 17.51 & 17.05 \\
\hline$(9,4)$ & 1120.70 & 1124.94 & 1122.86 & 22.60 & 26.44 & 23.23 \\
\hline$(8,4)$ & 1126.99 & 1131.44 & 1128.93 & 19.20 & 24.68 & 23.54 \\
\hline$(7,6)$ & 1148.25 & 1153.36 & 1149.52 & 23.81 & 25.32 & 24.23 \\
\hline$(8,6)$ & 1203.30 & 1205.06 & 1204.05 & 24.20 & 26.44 & 24.81 \\
\hline$(9,5)$ & 1272.35 & 1276.36 & 1273.79 & 28.66 & 30.64 & 28.71 \\
\hline$(8,7)$ & 1305.55 & 1309.52 & 1307.86 & 24.31 & 26.50 & 25.17 \\
\hline
\end{tabular}
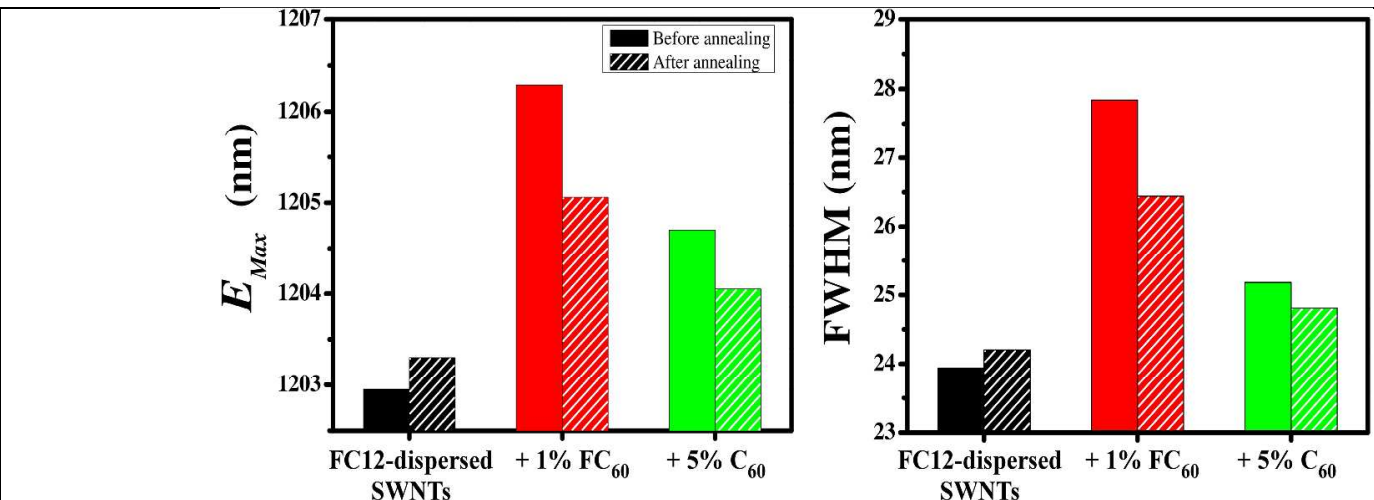

Figure S19. (a) Peak center $\left(E_{M a x}\right)$ positions and (b) FWHM values of $(8,6)$-SWNTs before (solidd) and after (hatched) $12 \mathrm{hr}$ annealing at $55^{\circ} \mathrm{C}$ for FC12-wrapped SWNTs, and molar mixtures of $1 \% \mathrm{FC}_{60}+99 \% \mathrm{FC} 12$ and 5\% $\mathrm{C}_{60}+95 \% \mathrm{FC} 12$, according to the deconvolution results shown in Figure S18 and Table S19. 


\section{F. Demonstration and quantification of $\mathrm{FC}_{60}$ incorporation within $\mathrm{FC12}$ helices}

In order to qualitatively establish $\mathrm{FC}_{60}$ incorporation within the $\mathrm{FC} 12$ helix, one needs to eliminate the extra amount of $\mathrm{FC}_{60}$ and $\mathrm{FC} 12$ solubilized in toluene. To achieve this, toluene SWNT dispersions with a molar mixture of $99 \% \mathrm{FC} 12$ and $1 \% \mathrm{FC}_{60}$ (herein referred as $1 \% \mathrm{FC}_{60}$ ) and $100 \%$ FC12, both of which were previously annealed for 12 hours at $55{ }^{\circ} \mathrm{C}$ (see SI Section E) were centrifuged at $100 \mathrm{Kg}$ for 1 hour to precipitate the majority of suspended nanotubes. The SWNT precipitates were washed multiple times with fresh toluene to ensure that all luminescence from the excess FC12 were removed in the decanted liquids. Subsequently, the SWNT precipitates were re-dispersed in toluene (via a bath sonication for 5 minutes) to afford a homogenous dispersion. The NIR absorption (Figure S20) and fluorescence spectra (Figure S21) verify $\mathrm{FC}_{60}$ incorporation based on the characteristic red-shift and strong SWNT PL quenching, respectively, with respect to $100 \%$ FC12-dispersed SWNT sample.

To quantitatively assess the percentage of $\mathrm{FC}_{60}$ incorporation, the re-dispersed SWNT absorption spectra of both $\mathrm{FC} 12$ and $1 \% \mathrm{FC}_{60}$ dispersions were first normalized at $450 \mathrm{~nm}$ (absorption maximum for flavin) and then subtracted from each other (i.e. $1 \% \mathrm{FC}_{60}$ mixture minus the pure FC12 wrapped nanotubes). Figure 5b in the main text and more specifically Figure S22 shows the subtraction spectrum (in blue) in comparison to the absorption spectrum of PCBM (in green). For comparison purposes, the subtraction spectrum was multiplied 15 times in order to exhibit the same optical density at $331 \mathrm{~nm}$ with a dilute PCBM solution in toluene. The two characteristic peaks of PCBM (i.e. 435 and $700 \mathrm{~nm}$ ) are clearly discernable in Figures S22d\&c, respectively. The lower energy peak, associated with the $\mathrm{S}_{0}$ to $\mathrm{S}_{1}$ transition, is strongly affected by charge transfer and exhibits a $6 \mathrm{~nm}$ redshift, which is in agreement with the SWNT-observed redshifts, discussed in SI Section E. The higher energy peak at $435 \mathrm{~nm}$ remains unaffected from $\mathrm{CT}$ and was chosen to estimate the amount of $\mathrm{FC}_{60}$ incorporation within the helix. For this, one needs to assume similar extinction coefficient for the $435 \mathrm{~nm}$ peak for both pure PCBM (in green) and the subtraction spectrum (in blue) in Figure S22. By multiplying the subtraction spectrum 41 times, the optical density of its $435 \mathrm{~nm}$ peak (background excluded) matches that of the dilute PCBM solution (background excluded) (data not shown). Based on this, the $\mathrm{FC}_{60}$ incorporation can be estimated to $c a .2 \%$ (i.e. 100/41), which is roughly double that of the starting $1 \% \mathrm{FC}_{60}$ molar ratio. 


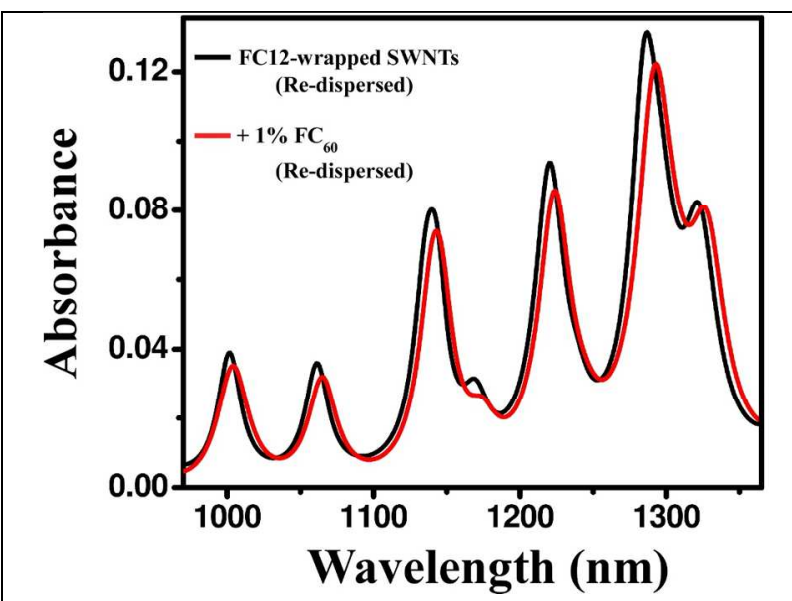

Figure S20. NIR absorption spectra of toluenedispersed SWNTs dispersed in either pure FC12 (black) or a molar mixture of $99 \% \mathrm{FC} 12$ and $1 \% \mathrm{FC}_{60}$ (red) that was previously precipitated and copiously washed with excess of toluene to remove all unbound FC12 and $\mathrm{FC}_{60}$ and re-dispersed in a similar volume of fresh toluene.

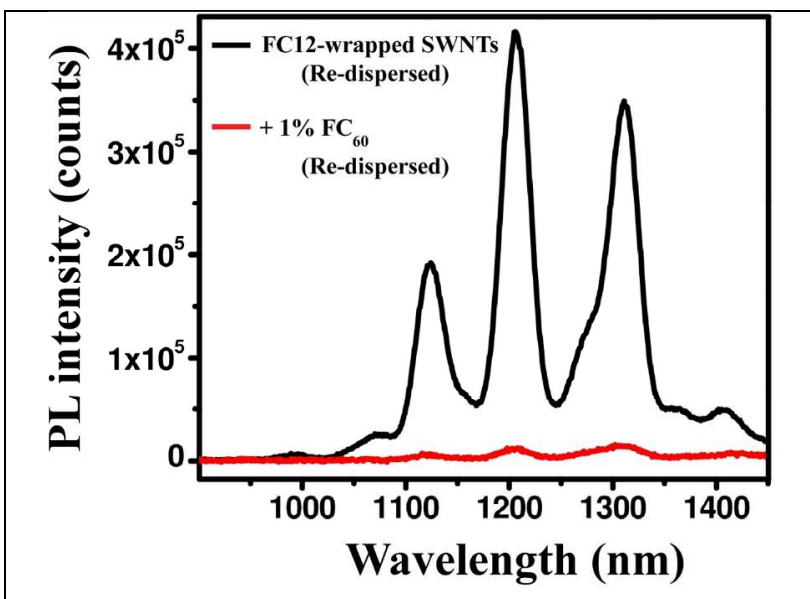

Figure S21. PL spectra of toluene-dispersed SWNTs dispersed in either pure FC12 (black) or a molar mixture of $99 \% \mathrm{FC} 12$ and $1 \% \mathrm{FC}_{60}$ (red) that was previously precipitated and copiously washed with excess of toluene to remove all unbound $\mathrm{FC} 12$ and $\mathrm{FC}_{60}$ and re-dispersed in a similar volume of fresh toluene. Excitation wavelength at $734 \mathrm{~nm}$.

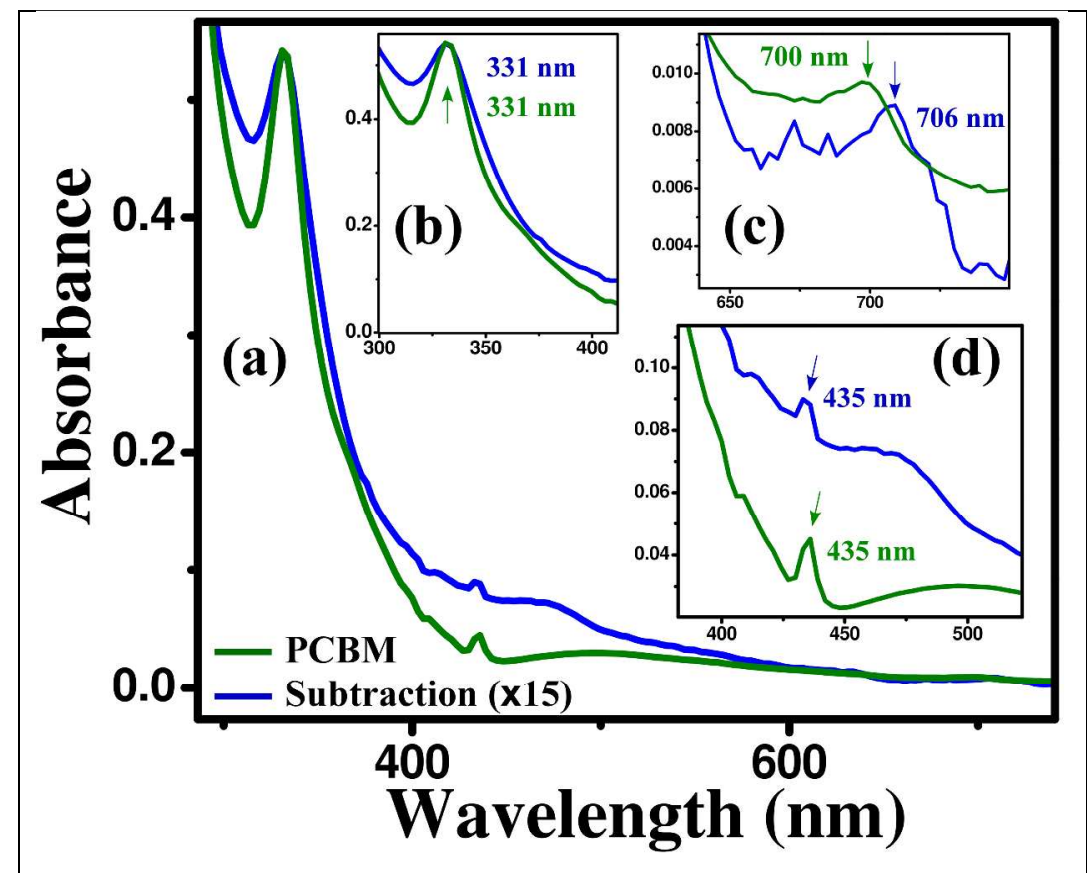

Figure S22. (a) Comparison of UV/Vis spectrum of PCBM (green) with the spectral subtraction of $1 \% \quad \mathrm{FC}_{60}$ mixture minus the pure $\mathrm{FC} 12$ wrapped nanotubes (from Figure $5 \mathrm{~b}$ of the main text). Insets (b), (c) and (d) enlarges the spectra around the 331,435 and $700 \mathrm{~nm}$ regions. 


\section{G. Spectroscopic changes in FC12-wrapped SWNTs with increasing incorporation of $\mathbf{F C}_{60}$}

As witnessed in SI Sections $\mathrm{E}$ and $\mathrm{F}$, the incorporation of $\mathrm{FC}_{60}$ within the $\mathrm{FC} 12$ helix induces: (i) a substantial redshift in the $E_{11}{ }^{S}$ absorption maxima; (ii) linewidth increase (in FWHM); and (iii) substantial PL quenching for all dispersed semiconducting SWNTs. In this section we explore the effect of higher $\mathrm{FC}_{60}$ concentration on the aforementioned three spectroscopic signatures. Attention was given to the production of stable suspensions. Upon closer observation of the dispersion stability of these $\mathrm{FC} 12 / \mathrm{FC}_{60}$ SWNT suspensions along with background increase (due to aggregation-induced scattering) and associated linewidth broadening in their Vis-NIR absorption spectra, incorporation of $\mathrm{FC}_{60}$ in excess of $3 \%$ results in severe nanotube aggregation. Moreover, annealing these dispersions at $55{ }^{\circ} \mathrm{C}$ for $c a .12$ hours contributed in substantial aggregations for $\mathrm{FC}_{60}$ incorporation in excess of $1 \%$. Based on that, no annealing results are presented in this section.

Figures S23a\&b illustrates the NIR absorbance and the $734 \mathrm{~nm}$ excitation PL spectra (which is resonant to $(8,6)$-SWNTs) for $0,1,2$ and $3 \%$ molar ratio of $\mathrm{FC}_{60}$ incorporation within $\mathrm{FC} 12$ mixtures used for SWNT dispersion. Following a similar spectral deconvolution as in the case of Figure S18 (deconvolution not shown), the $E_{11}^{S}$ transitions of various $(n, m)$-SWNTs experience a steady increase with higher $\mathrm{FC}_{60}$ content in both absorption maxima and FWHMs, as indicated in Table S20 \& S21 for both absorption and PL peaks, respectively. The linear increase in $E_{11} S$ absorption maxima red-shift with rising $\mathrm{FC}_{60}$ content (i.e. $0,1,2 \& 3 \%$ ) is clearly illustrated in Figure S24. Similarly, with higher $\mathrm{FC}_{60}$ content, the PL intensity progressively decreases for all dispersed nanotubes as shown in Figure S23b and the corresponding PLE maps of Figure S25 (note the decreasing magnitude of the colored $z$-scale bar, depicted at the right side of each map).

Increasing incorporation of $\mathrm{FC}_{60}$ induces a greater increase in redshift for absorption as opposed to PL maxima. In addition, all $(n, m)$-SWNT PL maxima are slightly blue-shifted (Table S21) with respect to the absorption maxima shown in Table S20. Both these indications point to the fact that SWNT emission originates from nanotubes segments that contain lesser $\mathrm{FC}_{60}$ incorporation and that segments with substantial $\mathrm{FC}_{60}$ content are heavily quenched.

In order to address a possible concern on whether the observed redshift in Figure S24 originates from $\mathrm{FC}_{60}$ incorporation, as opposed to $\mathrm{SWNT}$ aggregation, we gradually evaporated (by $\mathrm{N}_{2}$ bubbling) the toluene solvent of a dilute FC12-wrapped SWNTs and recorded the resulting VisNIR absorption spectra As shown in Figure S26a, the continuous surge in baseline slope (originating from enhanced light scattering) indicates the gradual increase in SWNT aggregation. Upon successive deconvolution, a negligible $E_{11}{ }^{S}$ redshift was observed and plotted as a function of increasing OD absorption at $920 \mathrm{~nm}$ (i.e. which lies between $E_{11}^{S}$ and $E_{22}^{S}$ manifolds and is devoid of SWNTs) (Figure S26b). This demonstrates that the observed red-shift in Figure S24 is not due to aggregation but rather due to increased $\mathrm{FC}_{60}$ incorporation. 


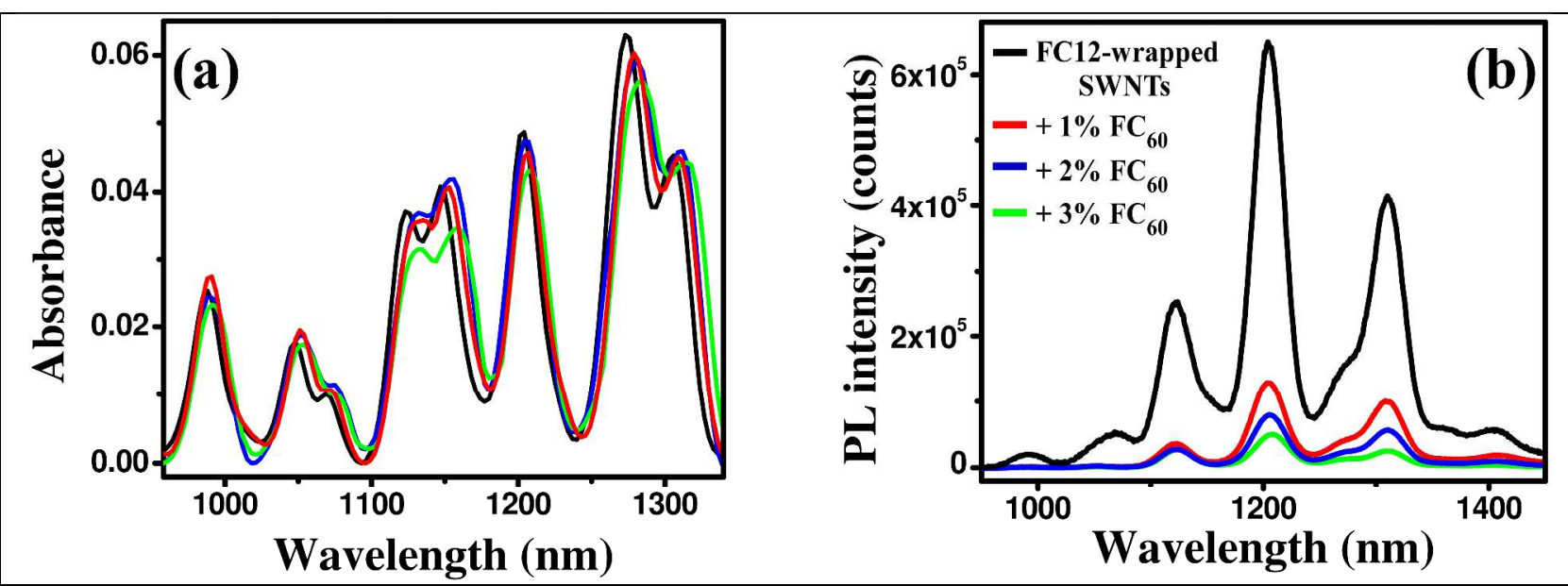

Figure S23. Comparison of NIR absorption (a) and PL (b) spectra of FC12-dispersed SWNTs in toluene with increasing molarity of $\mathrm{FC}_{60}$ (i.e. $0,1,2$ and $\left.3 \%\right)(734 \mathrm{~nm}$ excitation wavelength).

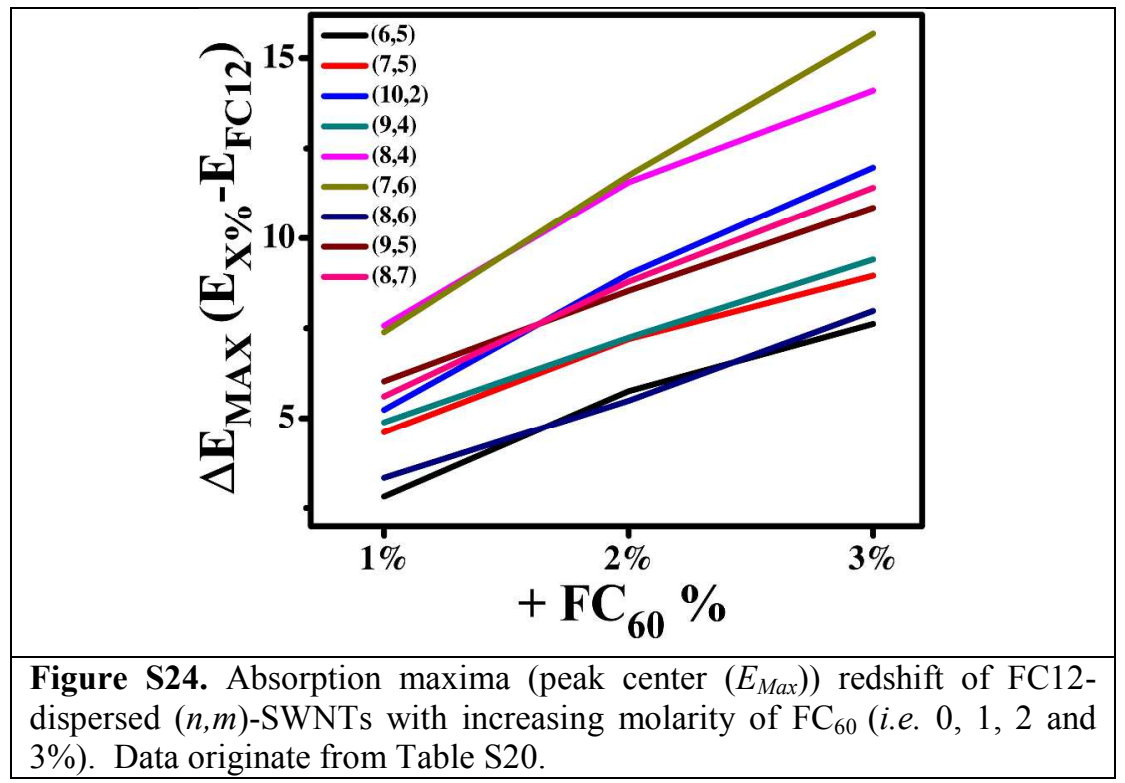


Table S20. $E^{S}{ }_{l l}$ position and FWHM comparison derived from the NIR absorption spectra of FC12-dispersed (n,m)SWNTs with increasing molarity of $\mathrm{FC}_{60}$ (i.e. 0, 1, 2 and 3\%) (from deconvolution of Figure S23a as described in SI Section E).

\begin{tabular}{|c|c|c|c|c|c|c|c|c|}
\hline \multirow[b]{2}{*}{$\begin{array}{c}\text { Nanotube } \\
\text { assignments } \\
(\mathrm{n}, \mathrm{m})\end{array}$} & \multicolumn{4}{|c|}{ Abs. $E_{\text {MAX }}(\mathrm{nm})$} & \multicolumn{4}{|c|}{ Abs. FWHM (nm) } \\
\hline & $\begin{array}{c}\text { FC12- } \\
\text { wrapped } \\
\text { SWNTs }\end{array}$ & $\begin{array}{l}+1 \% \\
\text { FC60 }\end{array}$ & $\begin{array}{l}+2 \% \\
\text { FC60 }\end{array}$ & $\begin{array}{l}+3 \% \\
\text { FC60 }\end{array}$ & $\begin{array}{c}\text { FC12- } \\
\text { wrapped } \\
\text { SWNTs }\end{array}$ & $\begin{array}{l}+1 \% \\
\text { FC60 }\end{array}$ & $\begin{array}{l}+2 \% \\
\text { FC60 }\end{array}$ & $\begin{array}{r}+3 \% \\
\text { FC60 }\end{array}$ \\
\hline$(6,5)$ & 987.0 & 989.8 & 992.7 & 994.6 & 21.1 & 23.6 & 25.8 & 26.5 \\
\hline$(7,5)$ & 1047.1 & 1051.7 & 1054.3 & 1056.1 & 19.7 & 22.8 & 24.7 & 27.6 \\
\hline$(10,2)$ & 1069.8 & 1075.0 & 1078.8 & 1081.7 & 14.2 & 18.2 & 19.9 & 20.2 \\
\hline$(9,4)$ & 1120.7 & 1125.6 & 1127.9 & 1130.1 & 22.5 & 27.1 & 28.8 & 29.6 \\
\hline$(8,4)$ & 1126.5 & 1134.1 & 1138.1 & 1140.6 & 19.1 & 25.6 & 26.2 & 30.6 \\
\hline$(7,6)$ & 1148.3 & 1155.6 & 1160.0 & 1163.9 & 23.2 & 26.7 & 27.6 & 28.3 \\
\hline$(8,6)$ & 1203.0 & 1206.3 & 1208.4 & 1210.9 & 23.9 & 27.8 & 28.7 & 29.8 \\
\hline$(9,5)$ & 1272.8 & 1278.8 & 1281.3 & 1283.6 & 28.8 & 31.7 & 34.3 & 35.2 \\
\hline$(8,7)$ & 1306.2 & 1311.8 & 1315.0 & 1317.6 & 24.6 & 27.4 & 28.7 & 29.4 \\
\hline
\end{tabular}

Table S21. $E_{11}^{S}$ position and FWHM comparison derived from the NIR emission spectra (excitation at $734 \mathrm{~nm}$ ) of FC12-dispersed $(n, m)$-SWNTs with increasing molarity of $\mathrm{FC}_{60}$ (i.e. 0, 1, 2 and 3\%) (from deconvolution of Figure $\mathrm{S} 23 \mathrm{~b}$, as described in SI Section E).

\begin{tabular}{|c|c|c|c|c|c|c|c|c|}
\hline \multirow{2}{*}{$\begin{array}{c}\text { Nanotube } \\
\text { assignments } \\
(\mathbf{n}, \mathbf{m})\end{array}$} & $\begin{array}{c}\text { FC12- } \\
\text { wrapped } \\
\text { SWNTs }\end{array}$ & $\begin{array}{c}+\mathbf{1} \% \\
\text { FC60 }\end{array}$ & $\begin{array}{c}+\mathbf{2} \% \\
\text { FC60 }\end{array}$ & $\begin{array}{c}\mathbf{+ 3} \% \\
\text { FC60 }\end{array}$ & $\begin{array}{c}\text { FC12- } \\
\text { wrapped } \\
\text { SWNTs }\end{array}$ & $\begin{array}{c}+\mathbf{+} \% \\
\text { FC60 }\end{array}$ & $\begin{array}{c}+\mathbf{2} \% \\
\text { FC60 }\end{array}$ & $\begin{array}{c}+\mathbf{3} \% \\
\text { FC60 }\end{array}$ \\
\hline $\mathbf{( 9 , 4 )}$ & 1122.3 & 1122.5 & 1122.9 & 1123.5 & 22.0 & 22.5 & 23.3 & 24.4 \\
\hline $\mathbf{8 , 6})$ & 1204.6 & 1204.6 & 1205.7 & 1207.7 & 28.6 & 28.8 & 29.7 & 30.0 \\
\hline $\mathbf{( 9 , 5 )}$ & 1273.0 & 1273.0 & 1273.6 & 1274.6 & 24.1 & 24.5 & 24.6 & 25.0 \\
\hline$(\mathbf{8}, 7)$ & 1308.9 & 1309.5 & 1309.7 & 1310.0 & 33.6 & 33.8 & 34.0 & 34.6 \\
\hline
\end{tabular}



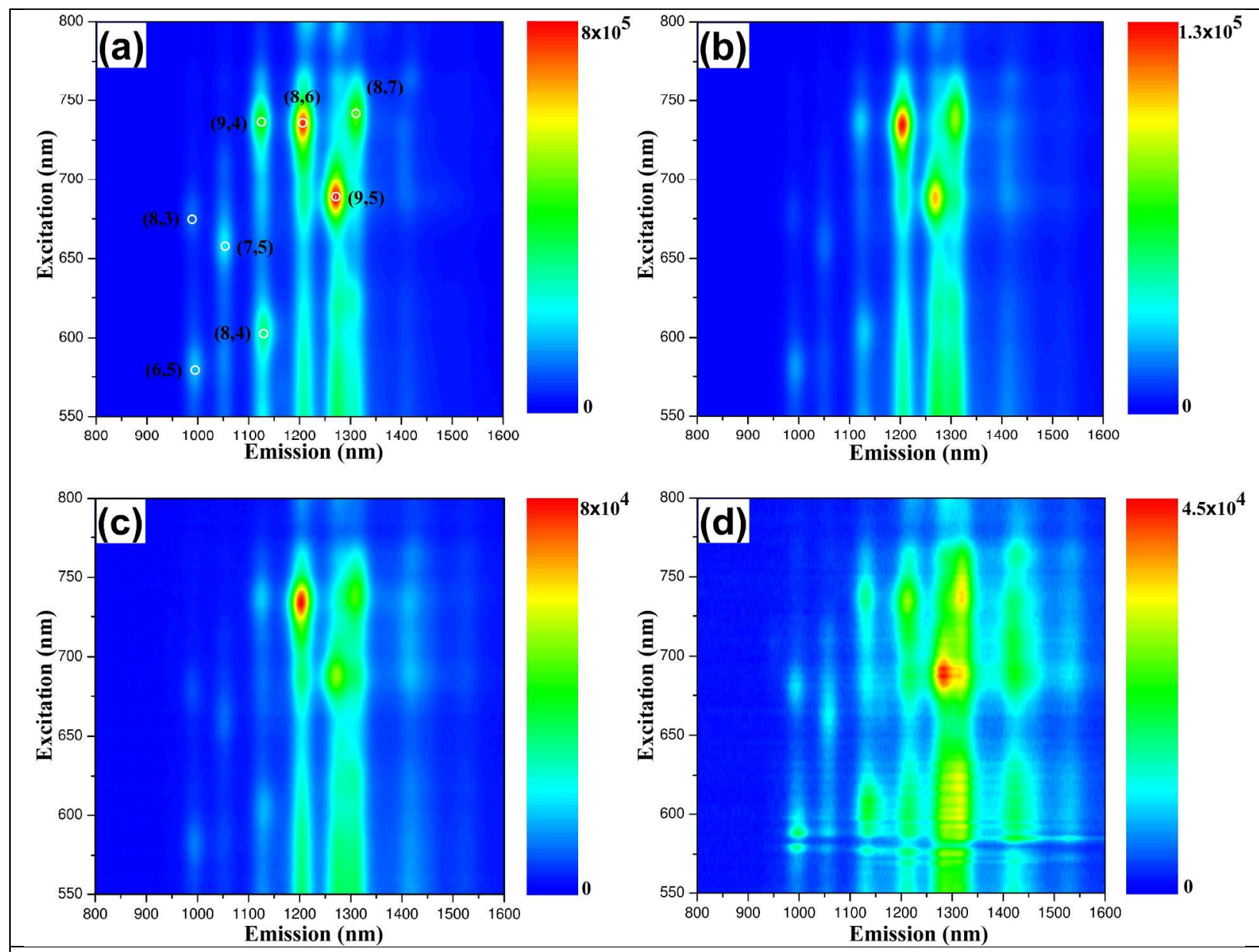

Figure S25. PLE maps of FC12-dispersed $(n, m)$-SWNTs with increasing molarity of $\mathrm{FC}_{60}$ (i.e. $0 \%$ (a), 1\% (b), $2 \%$ (c) and $3 \%($ d) $)$. Numbers in parentheses and white circles indicate the $(n, m)$ chiral indices and peak positions respectively for the observed sem-SWNTs. Increasing $\mathrm{FC}_{60}$ content decreases the magnitude of PL intensity as indicated by the colored Z-scale bar on the right side of each map.
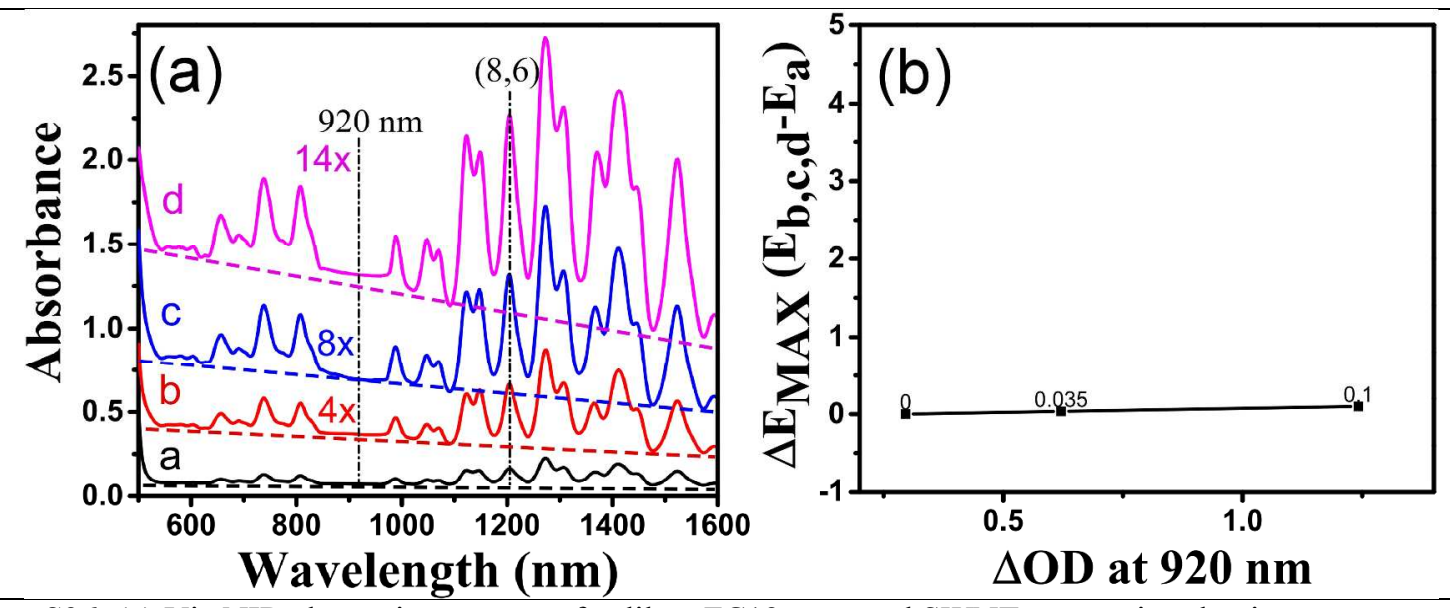

Figure S26. (a) Vis-NIR absorption spectra of a dilute FC12-wrapped SWNT suspension that is concentrated 4, 8 and 14 times in order to induce nanotube aggregation, as evident by the increasing slope (dashed line). (b) Such aggregation induces minite redshifts in the $E_{11}^{S}$ absorption maxima $\left(\mathrm{E}_{\mathrm{MAX}}(\mathrm{nm})\right)$, as expressed by the $\Delta \mathrm{E}_{\mathrm{MAX}} v s$. OD changes $(\Delta \mathrm{OD})$ at $920 \mathrm{~nm}$. 


\section{H. Transient spectroscopy of SWNTs wrapped with FC12 and a 3/97\% molar mixture of $\mathrm{FC}_{60} / \mathrm{FC12}$}

Pump-probe ultrafast transient absorption spectroscopy was carried out using a Helios femtosecond transient absorption spectrometer (Ultrafast Systems LLC, Sarasota, FL, USA) coupled to a laser setup that has been previously described. ${ }^{8}$ The optical density of the samples was adjusted to approximately $0.1-0.5$ at the excitation wavelength in a $2 \mathrm{~mm}$ path length cuvette. The excitation was $690 \mathrm{~nm}$ close to $\mathrm{E}_{22} \mathrm{~s}$ of $(8,6)-\mathrm{SWNT}$ (i.e. $734 \mathrm{~nm}$ ) in toluene. The pump laser had an energy of $2 \mu \mathrm{J} /$ pulse focused on a $1 \mathrm{~mm}$ diameter spot, which corresponds to a laser intensity of $\mathrm{ca} .6 \times 10^{14}$ photons $/ \mathrm{cm}^{2}$. The integrity of the samples was assayed by taking steady-state absorption spectra before and after laser excitation. The FC12-dispersed SWNTs and $3 \% \mathrm{FC}_{60}$ solutions were prepared as explained previously in Section-G of SI and annealed for limited time (see below) in $55{ }^{\circ} \mathrm{C}$. As already discussed in Section G, annealing of SWNTs dispersions containing in excess of $1 \% \mathrm{FC}_{60}$ results in substantial SWNT aggregation. However, in order to benefit from better $\mathrm{FC}_{60}$ partition within the $\mathrm{FC} 12$ helix we employed a short-term annealing ( $c a .2$ hours at $55{ }^{\circ} \mathrm{C}$ ) that prevented aggregation. Prior to obtaining transient absorption results, the sample was sonicated for $c a .30 \mathrm{~min}$ in a low intensity bath sonication at room temperature to ensure SWNT dispersion homogeneity.

Figure S27 shows transient absorption maps obtained for (a) FC12-wrapped SWNTs, (b) 3\% $\mathrm{FC}_{60}$ SWNTs in toluene. The transient absorption spectra for selected delay times are shown in Figure S28. Similar redshifts and FWHM broadening are witnessed as in the case of NIR absorption results of SI Section-G.

The decay curves probed at $1200 \mathrm{~nm}$ (resonant to $(8,6)$-SWNTs) are displayed in Figure 7 of the main text and are plotted together with exponential fits. The transient absorption rise time for both solutions is approximately $0.5 \mathrm{ps}$ but the $\mathrm{FC}_{60}$ containing SWNTs decays more rapidly on the picoseconds time scale compared to $\mathrm{FC}_{12}$-wrapped SWNTs. This suggests the presence of an additional quenching pathway provided by the proximal $\mathrm{C}_{60}$ moiety. Transient absorption decay spectrum also shows existence of a slow component which has an even slower decay for $\mathrm{FC}_{60}$ containing dispersed SWNTs revealing of photon-induced charge separation.

The time-dependent absorption data were fit with the following equation

$$
A b s(\tau)=A *\left(1+\operatorname{erf}\left(\frac{\tau-\tau_{o}}{\tau_{r}}\right)\right) *\left(e^{\frac{\tau-\tau_{O}}{\tau_{1}}}+b * e^{\frac{\tau-\tau_{o}}{\tau_{2}}}\right),
$$

where $\tau_{o}$ is time-zero, $\tau_{r}$ is the overall instrument response time, $\tau_{1}$ and $\tau_{2}$ are the exponential decay constants, $A$ sets the overall absorption, and $b$ is the relative contribution of the slow component compared to the fast component. 

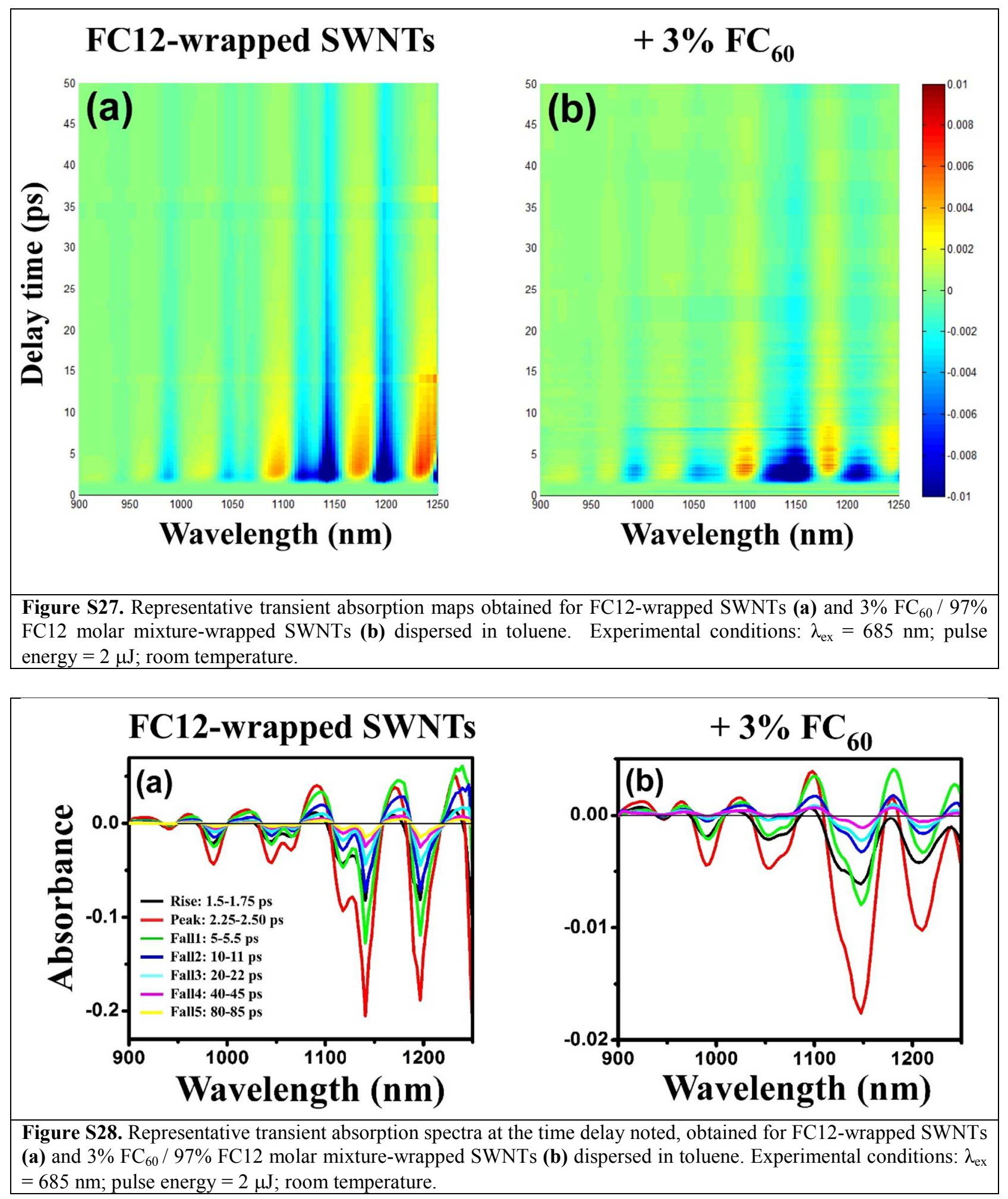


\section{Cited References}

(1) Mulliken, R.S., Person, W.B. J. Mol. Struct. 1971, 10, 155.

(2) Kampar, E.; Neilands, O. Russ. Chem. Rev. 1986, 55, 637-651.

(3) Bonetti, C.; Mathes, T.; van Stokkum, I. H. M.; Mullen, K. M.; Groot, M.-L.; van Grondelle, R.; Hegemann, P.; Kennis, J. T. M. Biophys. J. 2008, 95, 4790-4802.

(4) Kubota, L. T.; Gorton, L.; Roddick-Lanzilotta, A.; McQuillan, A. J. Bioelectrochemistry Bioenerg. 1998, 47, 39-46.

(5) Kottke, T.; Batschauer, A.; Ahmad, M.; Heberle, J. Biochemistry 2006, 45, 2472-2479.

(6) Martin, C. B.; Tsao, M. L.; Hadad, C. M.; Platz, M. S. J. Am. Chem. Soc. 2002, 124, $7226-7234$.

(7) Luo, Z.; Pfefferle, L. D.; Haller, G. L.; Papadimitrakopoulos, F. J. Am. Chem. Soc. 2006, 128, 15511-15516.

(8) Ilagan, R. P.; Christensen, R. L.; Chapp, T. W.; Gibson, G. N.; Pascher, T.; Polívka, T.; Frank, H. A. J. Phys. Chem. A 2005, 109, 3120-3127. 